\title{
gु \\ Masses in graphenelike two-dimensional electronic systems: Topological defects in order parameters and their fractional exchange statistics
}

\author{
Shinsei Ryu, ${ }^{1}$ Christopher Mudry, ${ }^{2}$ Chang-Yu Hou, ${ }^{3}$ and Claudio Chamon ${ }^{3}$ \\ ${ }^{1}$ Department of Physics, University of California, Berkeley, California 94720, USA \\ ${ }^{2}$ Condensed Matter Theory Group, Paul Scherrer Institut, CH-5232 Villigen PSI, Switzerland \\ ${ }^{3}$ Physics Department, Boston University, Boston, Massachusetts 02215, USA
}

(Received 9 September 2009; published 18 November 2009)

\begin{abstract}
We classify all possible 36 gap-opening instabilities in graphenelike structures in two dimensions, i.e., masses of Dirac Hamiltonian when the spin, valley, and superconducting channels are included. These 36 order parameters break up into 56 possible quintuplets of masses that add in quadrature and hence do not compete and thus can coexist. There is additionally a sixth competing mass, the one added by Haldane to obtain the quantum Hall effect in graphene without magnetic fields, which breaks time-reversal symmetry and competes with all other masses in any of the quintuplets. Topological defects in these five-dimensional order parameters can generically bind excitations with fractionalized quantum numbers. The problem simplifies greatly if we consider spin-rotation invariant systems without superconductivity. In such simplified systems, the possible masses are only 4 and correspond to the Kekule dimerization pattern, the staggered chemical potential, and the Haldane mass. Vortices in the Kekule pattern are topological defects that have Abelian fractional statistics in the presence of the Haldane term. We calculate the statistical angle by integrating out the massive fermions and constructing the effective field theory for the system. Finally, we discuss how one can have generically non-Landau-Ginzburg-type transitions with direct transitions between phases characterized by distinct order parameters.
\end{abstract}

DOI: 10.1103/PhysRevB.80.205319

PACS number(s): 73.20.Mf, 05.30.Pr, 71.10.-w

\section{INTRODUCTION}

Many of the physical properties of graphene are captured by a one-band tight-binding electronic Hamiltonian with uniform, real-valued, and nearest-neighbor hopping amplitude whereby (i) electron-electron interactions are ignored, (ii) spin-orbit interactions are ignored, (iii) the electronic band structure is replaced by two conical dispersions centered about two nonequivalent points, the Dirac points, in the first Brillouin zone, and (iv) the coupling to electromagnetic external fields is governed by the minimal substitution. For instance, graphene displays an integer quantum Hall effect (IQHE) as a function of the applied bias voltage, ${ }^{1,2}$ and it shows a universal optical conductivity. ${ }^{3}$ Both these properties can be understood within the noninteracting electron picture.

Although most experiments observe the massless Dirac spectrum assumed in (iii), electronic instabilities in the form of single-particle spectral gaps (mass gaps in short) can be triggered by external perturbations such as some commensurate substrates, ${ }^{4}$ or large enough magnetic fields that can change the balance between the kinetic and the potential energy. ${ }^{5-7}$ In this paper we study a number of issues pertaining to Dirac fermions in two dimensions when a mass gap is opened in the fermionic spectrum by different nonvanishing order parameters. In particular, we shall study in great detail the simpler case when there is no superconducting instabilities and spin-rotation invariance is maintained, in which case there are only four possible masses. We derive in this simpler case the effective action when the massive fermions are integrated out and read from this action the fractional statistics of topological defects in the mass order parameters. We also present a complete classification of all possible masses (36 in total) in the general case where any spin, valley, and superconducting instabilities are permitted.

In the simpler spinless problem (or, more realistically, the problem when spin-rotation invariance is never broken), the four different masses that can be added to the twodimensional Dirac equation representing graphene are the following. One perturbation is a staggered chemical potential, taking values $+\mu_{\mathrm{s}}$ and $-\mu_{\mathrm{s}}$ in the two sublattices of the honeycomb lattice of graphene say. It opens a gap $2\left|\mu_{\mathrm{s}}\right|$ at the two Dirac points. ${ }^{8}$ A second mass gap $2|\eta|$ arises by adding directed next-nearest-neighbor hopping amplitudes in the presence of fluxes, but such that no net magnetic flux threads a hexagonal Wigner-Seitz unit cell of graphene say. This perturbation breaks time-reversal symmetry (TRS). ${ }^{9}$ Finally, a real-valued modulation of the nearest-neighbor hopping amplitude with a wave vector connecting the two Dirac points (i.e., a Kekulé dimerization pattern for graphene) also opens a gap $2|\Delta| . .^{10}$ This real-valued modulation of the nearest-neighbor hoppings is parametrized by the complex order parameter $\Delta=\operatorname{Re} \Delta+i \operatorname{Im} \Delta$ whose phase controls the angles of the dimerization pattern. This mass corresponds to two real masses $\operatorname{Re} \Delta$ and $\operatorname{Im} \Delta$, bringing the total number of real-valued masses that conserve the electron number and spin-rotation symmetry (SRS) to four.

If the order parameters $\mu_{\mathrm{s}}, \eta$, and $\Delta$ are not uniform but vary in space and contain topological textures, then midgap states in the massive Dirac spectrum can appear. Examples are static line defects at which $\mu_{\mathrm{s}}$ and $\eta$ change signs, ${ }^{11}$ and static point defects represented by vortices in the phase of $\Delta .{ }^{10}$ As occurs at a static domain wall in one-dimensional polyacetylene, ${ }^{12-14}$ a fractional electronic charge is exponentially localized in the vicinity of a static charge \pm 1 vortex in the phase of $\Delta .^{10}$ 


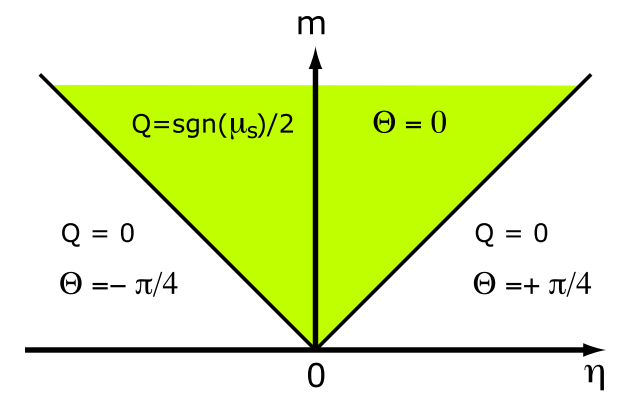

FIG. 1. (Color online) Phase diagram parametrized by the TRS mass $m$ and the TRS-breaking mass $\eta$. There are three regions delimited by the boundaries $|\eta|=m$, in each of which the spectral gap does not close. The boundaries $|\eta|=m$ are lines of critical points at which the spectral gap closes. When the vortices are screened by half of an axial gauge flux, they carry a fractional fermionic charge of $|Q|=1 / 2$ with the vanishing statistical phase $\Theta=0$ under pairwise exchange in regions for which TRS is weakly broken, i.e., the painted region $m>|\eta|$. Unit vortices are charge neutral but acquire the nonvanishing statistical phase $|\Theta|=\pi / 4$ under pairwise exchange in regions for which TRS is strongly broken, i.e., $|\eta|>m$ [see Eqs. (1.1a) and (1.1b)]. When the vortices are not screened by the axial gauge flux, the charge $Q$ acquires a dependence on the ratio of the chemical potential $\mu_{\mathrm{s}}$ and $m$, for $|\eta|<m$, and $Q$ vanishes for $|\eta|>m$. The statistics also depend on which phase one sits, but it is nonzero for any $\eta \neq 0$, and it is related to the value of the charge, as shown in Secs. VI and VII.

The value of the fractional charge that is bound to a vortex in the phase of $\Delta$ also depends on whether the vortex is dressed with a half flux of the axial vector potential $\boldsymbol{a}_{5}$ or not. ${ }^{15,16}$ When the axial gauge flux is absent (logarithmically confined case), the value of the charge can be tuned continuously as a function of the ratio $\mu_{\mathrm{s}} / m$, where $m:=\sqrt{|\Delta|^{2}+\mu_{\mathrm{s}}^{2}} \cdot{ }^{15,16}$ It is independent of the ratio $\mu_{\mathrm{s}} / m$ when the axial gauge half flux is present (deconfined case) for the charge is then pinned to the rational values $Q= \pm 1 / 2.15,16$ These values of the fractional charges persist as long as the magnitude of the TRS-breaking mass $|\eta|$ is smaller than the mass scale $m .^{15,16}$ There is a phase transition at $|\eta|=m$. For $|\eta|>m$ the fractional charge bound to the vortices vanishes. ${ }^{15,16}$

Just like the charge, the statistical phase $\Theta$ acquired upon the exchange of two vortices depends on whether the vortex in the phase of $\Delta$ is screened or not by the axial gauge flux. In this paper, we derive the statistical angle from the effective action obtained upon integrating out the massive fermions. (We thereby resolve conflicting claims about $\Theta$ in the literature. ${ }^{15,17,18}$ ) The statistical angle depends on the interplay between the magnitude of the TRS-breaking mass $\eta$ and the magnitude $m$ of the TRS masses. There are phase transitions at the lines $|\eta|=m$ depicted in Fig. 1 that separates regions dominated by the TRS-preserving masses and those dominated by the TRS-breaking mass $\eta$. The statistics $\Theta$ jumps for both the screened and unscreened vortices at the phase boundaries.

When unit vortices in $\Delta$ are screened by an axial gauge flux, they are deconfined. ${ }^{19}$ Their statistics is well defined in a dynamical sense and it takes universal values independent of the ratio $\mu_{\mathrm{s}}$ on both sides of the transition. We show that

$$
\Theta=0 \quad \text { when } m>|\eta|,
$$

and that

$$
\frac{\Theta}{\pi}=\operatorname{sgn}(\eta) Q^{2}=\frac{\operatorname{sgn} \eta}{4} \quad \text { when }|\eta|>m
$$

Along the lines $|\eta|=m$ in the zero-temperature phase diagram of Fig. 1, the gap in the Dirac spectrum vanishes. At criticality, the notion of point particles is moot and so is the question of their quantum numbers.

A remarkable complementarity has emerged. Defects carry either a fractional charge $Q= \pm 1 / 2$ but no fractional statistical phase when the breaking of TRS is not too strong $(|\eta|<m)$, or no fractional charge but a fractional statistics $\Theta / \pi= \pm 1 / 4$ when the breaking of TRS is dominant $(|\eta|>m)$.

When unit vortices in the order parameter $\Delta$ are not accompanied by an axial gauge flux, they are logarithmically confined. ${ }^{10}$ Although their statistics is not well-defined dynamically, it is nevertheless possible to create them and exchange them by external means. If so, both their charges and statistics acquire a dependence on all masses $\eta, \mu_{\mathrm{s}}$, and $\Delta$, which we compute analytically and test numerically here in this paper.

We then go beyond the simpler spinless case with only four masses, and we classify all 36 masses in the general case where any spin, valley, and superconducting instabilities are allowed. These 36 order parameters break up into 56 possible quintuplets of masses that add in quadrature (to a value $\mathrm{m}^{2}$ ) and thus do not compete with one another. The Haldane mass, the generalization of the $\eta$ mass above, competes with all the other 35 masses, and thus one has generically a quantum phase transition when $|\eta|=m$. We argue that these five-tuplets provide a rich playground for Landauforbidden continuous phase transitions. We discuss in the paper how any $U(1)$ order parameter in a five-tuplet can be assigned a conserved charge and supports topological defects in the form of vortices. A pair of U(1) order parameters in a five-tuplet is said to be dual if the vortices of one order parameter binds the charge of the other order parameter and vice versa. A continuous phase transition can then connect directly the two dual $\mathrm{U}(1)$ ordered phases through a confining-deconfining transition of their vortices.

This paper is organized as follows. We define the relevant continuum Dirac Hamiltonian and review its symmetries for the simpler problem with only four masses that encodes the competition between charge-density, bond-density, and integer-quantum-Hall instabilities at the Dirac (charge neutral) point of any graphenelike two-dimensional electronic system in Sec. II. We reveal a hidden non-Abelian structure of the field theory in Sec. III that plays an important role when deriving the charge and statistics of quasiparticles. The fermions are integrated in the background of these four order parameters and of the $\mathrm{U}(1) \times \mathrm{U}(1)$ gauge fields to leading order in a gradient expansion in Sec. IV. The effective lowenergy and long-wavelength interacting field theory thereby obtained is a Anderson-Higgs-Chern-Simons field theory for bosonic fields: two U(1) gauge fields and one phase field. The induced fractional fermion number and the induced frac- 
tional Abelian statistical phase in the Anderson-HiggsChern-Simons field theory of Sec. IV are computed in Sec. V and Sec. VI, respectively. The numerical calculation of the fractional charges and statistical phases within a singleparticle (mean-field) approximation that violates the $\mathrm{U}(1) \times \mathrm{U}(1)$ gauge symmetry is presented in Sec. VII. A microscopic (lattice) model sharing the same $\mathrm{U}(1) \times \mathrm{U}(1)$ gauge symmetry and low-energy long-wavelength particle content as the Anderson-Higgs-Chern-Simons field theory is constructed in Sec. VIII. Either by enlarging the particle content of the lattice model from Sec. VIII or by allowing additional magnetic, spin-orbit, or superconductivity instabilities to compete with the charge-density, bond-density, and integer-quantum-Hall instabilities in graphenelike twodimensional systems, we are lead to a classification presented in Sec. IX of all 36 competing orders of a Dirac Hamiltonian represented by 16-dimensional Dirac matrices that encodes the quantum dynamics of electrons constrained to a two-dimensional space, as occurs in graphene at the charge neutral point say. We conclude in Sec. X and relegate some intermediary steps to the Appendixes.

\section{HAMILTONIAN AND SYMMETRIES: SPINLESS CASE WITH FOUR MASSES}

The continuum model under consideration in this paper is defined by the second-quantized planar Hamiltonian $\hat{H}:=\int d^{2} \boldsymbol{r} \hat{\mathcal{H}}$, where ${ }^{20}$

$$
\begin{gathered}
\hat{\mathcal{H}}:=\hat{\mathcal{H}}_{0}+\hat{\mathcal{H}}_{\text {gauge }}+\hat{\mathcal{H}}_{\text {scalar }}, \\
\hat{\mathcal{H}}_{0}:=\hat{\psi}^{\dagger} \boldsymbol{\alpha} \cdot(-i \boldsymbol{\partial}) \hat{\psi}, \\
\hat{\mathcal{H}}_{\text {gauge }}:=\hat{\psi}^{\dagger} \boldsymbol{\alpha} \cdot\left(\boldsymbol{a}+\boldsymbol{a}_{5} \gamma_{5}\right) \hat{\psi}, \\
\hat{\mathcal{H}}_{\text {scalar }}:=\hat{\psi}^{\dagger}\left(|\Delta| \beta e^{i \theta \gamma_{5}}+\mu_{\mathrm{s}} R+i \eta \alpha_{1} \alpha_{2}\right) \hat{\psi} .
\end{gathered}
$$

The four components of the spinor-valued operator,

$$
\hat{\psi}(\boldsymbol{r})=\left(\begin{array}{c}
\hat{\psi}_{+\mathrm{A}}(\boldsymbol{r}) \\
\hat{\psi}_{+\mathrm{B}}(\boldsymbol{r}) \\
\hat{\psi}_{-\mathrm{B}}(\boldsymbol{r}) \\
\hat{\psi}_{-\mathrm{A}}(\boldsymbol{r})
\end{array}\right) \equiv\left(\hat{\psi}_{\ell}(\boldsymbol{r})\right),
$$

obey the equal-time fermion algebra,

$$
\begin{gathered}
\left\{\hat{\psi}_{\ell}(\boldsymbol{r}), \hat{\psi}_{\ell^{\prime}}^{\dagger}\left(\boldsymbol{r}^{\prime}\right)\right\}=\delta_{\ell, \ell^{\prime}} \delta\left(\boldsymbol{r}-\boldsymbol{r}^{\prime}\right), \\
\left\{\hat{\psi}_{\ell}^{\dagger}(\boldsymbol{r}), \hat{\psi}_{\ell^{\prime}}^{\dagger}\left(\boldsymbol{r}^{\prime}\right)\right\}=\left\{\hat{\psi}_{\ell}(\boldsymbol{r}), \hat{\psi}_{\ell^{\prime}}\left(\boldsymbol{r}^{\prime}\right)\right\}=0 .
\end{gathered}
$$

Representation (2.1b) is here fixed by the indices A and B that distinguish the two triangular sublattices of the honeycomb lattice and the indices + and - that distinguish the two inequivalent Dirac points (valleys) of graphene. With this choice, the four Dirac matrices $\alpha^{x} \equiv \alpha^{1}, \alpha^{y} \equiv \alpha^{2}$, $\alpha^{z} \equiv \alpha^{3} \equiv R$, and $\beta$ are defined by their four-dimensional chiral representation, ${ }^{21}$

$$
\begin{gathered}
\alpha:=\left(\begin{array}{cc}
\boldsymbol{\tau} & 0 \\
0 & -\boldsymbol{\tau}
\end{array}\right) \equiv \sigma_{3} \otimes \boldsymbol{\tau} \equiv\left(\alpha^{1}, \alpha^{2}\right), \\
\alpha^{3}:=\left(\begin{array}{cc}
\tau_{3} & 0 \\
0 & -\tau_{3}
\end{array}\right) \equiv \sigma_{3} \otimes \tau_{3} \equiv R, \\
\beta:=\left(\begin{array}{cc}
0 & \tau_{0} \\
\tau_{0} & 0
\end{array}\right) \equiv \sigma_{1} \otimes \tau_{0},
\end{gathered}
$$

where the $2 \times 2$ unit matrix $\tau_{0}$ and the three Pauli matrices $\tau_{1}, \tau_{2}$, and $\tau_{3}$ act on the sublattices indices (A and B) while the $2 \times 2$ unit matrix $\sigma_{0}$ and the three Pauli matrices $\sigma_{1}, \sigma_{2}$, and $\sigma_{3}$ act on the valley indices ( + and - ). The matrix,

$$
\gamma_{5} \equiv \gamma^{5}:=-i \alpha^{1} \alpha^{2} \alpha^{3}=\left(\begin{array}{cc}
\tau_{0} & 0 \\
0 & -\tau_{0}
\end{array}\right) \equiv \sigma_{3} \otimes \tau_{0},
$$

acts trivially on the sublattices indices while it acts nontrivially on the valley indices, i.e., $\left(1 \pm \gamma_{5}\right) / 2$ is a projector on the + and - valley indices, respectively. In $(3+1)$-dimensional space and time quantum electrodynamics, the eigenspaces of $\left(1 \pm \gamma_{5}\right) / 2$ define the chiral indices, a terminology that we shall also use in this paper. The external (background) real-valued fields $\boldsymbol{a}=\left(a_{1}, a_{2}\right), \boldsymbol{a}_{5}=\left(a_{51}, a_{52}\right)$, $|\Delta|, \theta \equiv-\arg \Delta, \mu_{\mathrm{s}}$, and $\eta$ are space- and time-dependent fields. Their microscopic interpretation is the following.

A strong uniform magnetic field (rotational of $\boldsymbol{a}$ ) is responsible for the IQHE in graphene. ${ }^{22}$ A vector field $\boldsymbol{a}_{5}$ encodes changes in the curvature (ripples) of graphene, ${ }^{23,24}$ and it can also encode defective coordination numbers at apical defects. ${ }^{25-27} \mathrm{~A}$ constant $\mu_{\mathrm{s}}$ realizes in graphene a staggered chemical potential and opens an electronic spectral gap. ${ }^{8} \mathrm{~A}$ constant $\eta$ realizes in graphene a directed next-nearestneighbor hopping amplitude without net magnetic flux through the Wigner-Seitz cell of the honeycomb lattice and it also opens an electronic spectral gap. ${ }^{9}$ A constant $\Delta$ realizes in graphene a Kekulé distortion of the nearest-neighbor hopping amplitude and, again, opens an electronic spectral gap. ${ }^{10}$ The four space- and time-independent $\operatorname{Re} \Delta, \operatorname{Im} \Delta, \mu_{\mathrm{s}}$, and $\eta$ exhaust all possible ways for the opening of a spectral gap in the single-particle spectrum of the kinetic Dirac kernel $\boldsymbol{\alpha} \cdot(-i \boldsymbol{\partial})$, as $\beta, \beta \gamma_{5}, R$, and $i \alpha^{1} \alpha^{2}$ generate the largest set of traceless and Hermitian $4 \times 4$ matrices that anticommutes with $\boldsymbol{\alpha} \cdot(-i \boldsymbol{\partial})$. The three masses $\operatorname{Re} \Delta, \operatorname{Im} \Delta$, and $\mu_{\mathrm{s}}$ are compatible, i.e., they open the gap $2 m$, where

$$
m:=\sqrt{|\Delta|^{2}+\mu_{\mathrm{s}}^{2}},
$$

for $\beta, \beta \gamma_{5}$, and $R$ anticommute pairwise. On the other hand, the mass $\eta$ competes with the mass $m$, as $i \alpha_{1} \alpha_{2}$ commutes with $\beta, \beta \gamma_{5}$, and $R$ (the competition between $\eta$ and $m$ leads to a phase transition when $|\eta|=m$, which shall be important in the discussion of fractional statistics in this paper). The fields $\boldsymbol{a}, \boldsymbol{a}_{5}, \Delta, \mu_{\mathrm{s}}$, and $\eta$ have also appeared in the context of (a) slave-boson treatments of antiferromagnetic spin-1/2 Heisenberg model on the square lattice in the $\pi$-flux phase, ${ }^{28-31}$ and (b) Anderson localization for electrons hopping on a square lattice with a flux of half a magnetic flux 
quantum per plaquette, i.e., the square lattice with $\pi$-flux phase. ${ }^{32-34}$

\section{Symmetries}

The model defined in Eq. (2.1a), (2.1b), (2.1c), (2.1d), and (2.1e) possesses a number of symmetry operations that we list below and utilize in the paper.

\section{Time-reversal symmetry}

In the Heisenberg representation,

$$
\hat{H}(t) \rightarrow \hat{H}(-t),
$$

under the anti-unitary transformation,

$$
\begin{gathered}
\hat{\psi}(\boldsymbol{r}, t) \rightarrow(T K \hat{\psi})(\boldsymbol{r},-t), \\
\boldsymbol{a}(\boldsymbol{r}, t) \rightarrow-\boldsymbol{a}(\boldsymbol{r},-t), \quad \eta(\boldsymbol{r}, t) \rightarrow-\eta(\boldsymbol{r},-t), \\
\boldsymbol{a}_{5}(\boldsymbol{r}, t) \rightarrow \boldsymbol{a}_{5}(\boldsymbol{r},-t), \quad \theta(\boldsymbol{r}, t) \rightarrow \theta(\boldsymbol{r},-t), \\
|\Delta|(\boldsymbol{r}, t) \rightarrow|\Delta|(\boldsymbol{r},-t), \quad \mu_{\mathrm{s}}(\boldsymbol{r}, t) \rightarrow \mu_{\mathrm{s}}(\boldsymbol{r},-t),
\end{gathered}
$$

where complex conjugation is represented by $K$ and

$$
T:=\beta \alpha^{1} \gamma_{5}=\left(\begin{array}{cc}
0 & \tau_{1} \\
\tau_{1} & 0
\end{array}\right) \equiv \sigma_{1} \otimes \tau_{1}=T^{t}
$$

is a unitary Hermitian (and thus symmetric) matrix. Transformation (2.4) realizes reversal of time in graphene for $T$ exchanges the two valleys while acting trivially on the sublattice indices. Moreover, transformation (2.4) realizes reversal of time for an effectively spinless single particle for $T$ is symmetric. Hamiltonian $\hat{H}$ is time-reversal symmetric and can be represented by real-valued matrix elements ${ }^{35}$ if all background fields are static while

$$
a_{1}=a_{2}=\eta=0 \text {. }
$$

\section{Sublattice symmetry}

Always in the Heisenberg representation,

$$
\hat{H}(t) \rightarrow-\hat{H}(t),
$$

under the unitary transformation

$$
\begin{gathered}
\hat{\psi}(\boldsymbol{r}, t) \rightarrow(R \hat{\psi})(\boldsymbol{r}, t), \\
\boldsymbol{a}(\boldsymbol{r}, t) \rightarrow \boldsymbol{a}(\boldsymbol{r}, t), \quad \eta(\boldsymbol{r}, t) \rightarrow-\eta(\boldsymbol{r}, t), \\
\boldsymbol{a}_{5}(\boldsymbol{r}, t) \rightarrow \boldsymbol{a}_{5}(\boldsymbol{r}, t), \quad \theta(\boldsymbol{r}, t) \rightarrow \theta(\boldsymbol{r}, t), \\
|\Delta|(\boldsymbol{r}, t) \rightarrow|\Delta|(\boldsymbol{r}, t), \quad \mu_{\mathrm{s}}(\boldsymbol{r}, t) \rightarrow-\mu_{\mathrm{s}}(\boldsymbol{r}, t),
\end{gathered}
$$

where

$$
R:=\alpha_{3}=\left(\begin{array}{cc}
\tau_{3} & 0 \\
0 & -\tau_{3}
\end{array}\right) \equiv \sigma_{3} \otimes \tau_{3}=R^{t}
$$

is a diagonal, unitary, and Hermitian matrix. Transformation (2.8) realizes in graphene the change of sign of the single- particle wave functions on every sites of the honeycomb lattice belonging to one and only one triangular sublattice. The single-particle eigenstates of the conserved Hamiltonian $\hat{H}$ obey the spectral symmetry (SLS) by which any singleparticle eigenstate $|\Psi\rangle$ with a nonvanishing energy eigenvalue $\varepsilon$ has the mirror eigenstate $R|\Psi\rangle$ with the nonvanishing energy eigenvalue $-\varepsilon$ if all background fields are static while

$$
\mu_{\mathrm{s}}=\eta=0 .
$$

\section{Continuous gauge symmetries}

We now turn to the continuous symmetries obeyed by the Dirac Hamiltonian (2.1a), (2.1b), (2.1c), (2.1d), and (2.1e) in the Heisenberg representation. To this end, we make use of

$$
0=\left[\gamma_{5}, \boldsymbol{\alpha}\right]=\left\{\gamma_{5}, \beta\right\}=\left[\gamma_{5}, R\right] .
$$

The commutators and anticommutator (2.11) imply that

$$
\hat{H}(t) \rightarrow \hat{H}(t)
$$

under the $\mathrm{U}(1) \otimes \mathrm{U}(1)$ local gauge transformation,

$$
\begin{gathered}
\hat{\psi} \rightarrow e^{i\left(\phi+\phi_{5} \gamma_{5}\right)} \hat{\psi}, \quad \boldsymbol{a} \rightarrow \boldsymbol{a}-\boldsymbol{\partial} \phi, \\
\boldsymbol{a}_{5} \rightarrow \boldsymbol{a}_{5}-\boldsymbol{\partial} \phi_{5}, \quad \theta \rightarrow \theta-2 \phi_{5}, \\
|\Delta| \rightarrow|\Delta|, \quad \mu_{\mathrm{s}} \rightarrow \mu_{\mathrm{s}}, \quad \eta \rightarrow \eta,
\end{gathered}
$$

generated by the two space- and time-dependent real-valued smooth functions $\phi$ and $\phi_{5}$. The microscopic origin of the global U(1) gauge symmetry generated by $\phi$ is conservation of the electron number in graphene. For planar graphene, the continuous global axial U(1) gauge symmetry generated by $\phi_{5}$ is broken as soon as the curvature of the tight-binding dispersion is accounted for so that the Dirac points are not anymore decoupled. We shall nevertheless impose the local axial U(1) gauge symmetry at the level of the approximation captured by the Dirac Hamiltonian (2.1a), (2.1b), (2.1c), (2.1d), and (2.1e) and see through its consequences in this paper. [We do provide a microscopic example of a lattice model that realizes the local axial $\mathrm{U}(1)$ gauge symmetry in Sec. VIII.]

\section{PATH INTEGRAL FORMULATION OF THE MODEL WITH FOUR MASSES}

For our purposes, it will be more convenient to trade the operator formalism for an effective partition function defined by integrating over the Dirac fermions in the background of the gauge fields $\boldsymbol{a}$ and $\boldsymbol{a}_{5}$ and of the scalar fields $\Delta, \mu_{\mathrm{s}}$, and $\eta$. We will demand that this effective theory captures the $\mathrm{U}(1) \otimes \mathrm{U}(1)$ local gauge symmetry (2.13). This is possible in odd-dimensional space and time ${ }^{36}$ for the Grassmann measure can be regularized without breaking the $\mathrm{U}(1) \otimes \mathrm{U}(1)$ local gauge symmetry of the Lagrangian. Of course, maintaining the $\mathrm{U}(1) \otimes \mathrm{U}(1)$ local gauge symmetry can only be achieved if the phase $\theta=-\arg \Delta$ of the Kekule background field $\Delta$ is also included as a dynamical field. For simplicity but without loss of generality as far as the computation of the 
charge quantum number and statistical phase are concerned, the masses $m$ and $\eta$ will be taken to be space- and timeindependent parameters, while $\Delta$ and $\mu_{\mathrm{s}}$ vary in space and time (with $m=\sqrt{|\Delta|^{2}+\mu_{\mathrm{s}}^{2}}$ constant) through $\theta \equiv-\arg \Delta$ and $\cos \alpha \equiv \mu_{\mathrm{s}} / m$. (For simplicity, we shall also focus on the case where $\mu_{\mathrm{s}}$ is also constant in space and time, with the exception of near the vortex core, where $\Delta \rightarrow 0$, so $\mu_{\mathrm{s}}$ has to adjust so as to keep $m$ constant.)

Thus, we seek the effective field theory defined by the Grassmann path integral,

$$
\begin{gathered}
Z_{m, \eta}\left[a_{\mu}, a_{5 \mu}, \theta, \alpha\right]:=\int \mathcal{D}[\bar{\psi}, \psi] \exp \left(i \int d^{3} x \mathcal{L}_{m, \eta}\right), \\
\mathcal{L}_{m, \eta}:=\bar{\psi}\left(\gamma^{\mu} i \partial_{\mu}-\gamma^{\mu} a_{\mu}-\gamma^{\mu} \gamma_{5} a_{5 \mu}-M_{m, \eta}\right) \psi,
\end{gathered}
$$

where we have also included the time components $a_{0}$ (TRS but SLS breaking) and $a_{50}$ (SLS but TRS breaking) of the $\mathrm{U}(1) \otimes \mathrm{U}(1)$ gauge fields to maintain space and time covariance. The independent Grassmann-valued fields over which the path integral is performed are the four-components spinors $\bar{\psi}$ and $\psi$. They depend on the contravariant three-vectors $x^{\mu}=(t, \boldsymbol{r}) \quad\left[\right.$ covariant three-vectors $\left.x_{\mu}=(t,-\boldsymbol{r})\right]$ and we will use the repeated summation convention $x^{\mu} y_{\mu}=x^{0} y^{0}-x^{1} y^{1}-x^{2} y^{2}$. We have defined the four gamma matrices,

$$
\gamma^{0}:=\beta, \quad \gamma^{1}:=\beta \alpha^{1}, \quad \gamma^{2}:=\beta \alpha^{2}, \quad \gamma^{3}:=\beta \alpha^{3},
$$

for which lowering and raising of the greek indices $\mu, \nu=0,1,2$ is achieved with the Lorentz metric $g_{\mu \nu}=\operatorname{diag}(1,-1,-1)$. The four matrices $\gamma^{0}, \gamma^{1}, \gamma^{2}$, and $\gamma^{3}$ obey the usual Clifford algebra in Minkowsky space in the chiral representation, i.e., $\gamma_{5}=i \gamma^{0} \gamma^{1} \gamma^{2} \gamma^{3}$ is diagonal. We have also defined the matrix,

$$
\begin{aligned}
& M_{m, \eta}:=m\left(n_{1} M_{1}+n_{2} M_{2}+n_{3} M_{3}\right)+\eta \gamma_{5} \gamma^{3}, \\
& M_{1}:=1, \quad M_{2}:=-i \gamma_{5}, \quad M_{3}:=\beta \alpha^{3} \equiv \gamma^{3},
\end{aligned}
$$

for which we do not distinguish upper and lower latin indices $a, b=1,2,3$ as they are contracted with the Euclidean metric $\delta_{\mathrm{ab}}=\operatorname{diag}(1,1,1)$. [Notice that because space and time is $(2+1)$ dimensional, we can use the gamma matrix $\gamma^{3}$ to open a spectral gap by taking $M_{3}=\gamma^{3}$.] The space and time dependencies in $M_{m, \eta}$ follow entirely from those of the phase $\arg \Delta$. Indeed, while the masses $\eta$ and $m$ are constant in space and time, the direction of the unit vector $\boldsymbol{n}$ with the three components,

$$
n_{1}:=\frac{|\Delta| \cos \theta}{m}, \quad n_{2}:=-\frac{|\Delta| \sin \theta}{m}, \quad n_{3}:=\frac{\mu_{\mathrm{s}}}{m},
$$

can vary in space and time.

The $\mathrm{U}(1) \otimes \mathrm{U}(1)$ local gauge symmetry (2.13) has become the invariance of the Lagrangian in Eq. (3.1a), (3.1b), (3.1c), and (3.1d) under the $\mathrm{U}(1) \otimes \mathrm{U}(1)$ local gauge transformation,

$$
\bar{\psi} \rightarrow \bar{\psi} e^{-i\left(\phi-\phi_{5} \gamma_{5}\right)}, \quad \psi \rightarrow e^{i\left(\phi+\phi_{5} \gamma_{5}\right)} \psi
$$

$$
\begin{gathered}
a_{\mu} \rightarrow a_{\mu}-\partial_{\mu} \phi, \quad a_{5 \mu} \rightarrow a_{5 \mu}-\partial_{\mu} \phi_{5}, \\
\theta \rightarrow \theta-2 \phi_{5} .
\end{gathered}
$$

In spite of appearances $\left[\bar{\psi} \psi \rightarrow \bar{\psi} \exp \left(2 i \phi_{5} \gamma_{5}\right) \psi\right]$, the Grassmann Jacobian induced by the $\mathrm{U}(1) \otimes \mathrm{U}(1)$ local gauge transformation (2.13) is unity and does not produce a quantum anomaly in $(2+1)$ dimensions (odd space-time dimension). ${ }^{36}$

We take advantage of the fact that $\bar{\psi}$ and $\psi$ are independent Grassmann integration variables to bring the algebra obeyed by the six matrices $\gamma^{\mu} \mu=0,1,2$ and $M_{a} a=1,2,3$ to a form that will simplify greatly the evaluation of the partition function (3.1a), (3.1b), (3.1c), and (3.1d). Under the nonunitary change of integration variable,

$$
\bar{\psi}=: \bar{\chi} \gamma_{5} \gamma^{3}, \quad \psi=: \chi,
$$

the partition function (3.1a), (3.1b), (3.1c), and (3.1d) becomes

$$
\begin{gathered}
Z_{m, \eta}\left[B_{\mu}, n_{\mathrm{a}}\right]=\int \mathcal{D}[\bar{\chi}, \chi] \exp \left(i \int d^{3} x \mathcal{L}_{m, \eta}\right), \\
\mathcal{L}_{m, \eta}=\bar{\chi}\left(\Gamma^{\mu} i \partial_{\mu}+\Gamma^{\mu} B_{\mu}-m n_{\mathrm{a}} \Sigma_{\mathrm{a}}-\eta\right) \chi,
\end{gathered}
$$

where the matrices

$$
\Gamma^{\mu}:=\gamma_{5} \gamma^{3} \gamma^{\mu}, \quad \Sigma_{\mathrm{a}}:=\gamma_{5} \gamma^{3} M_{\mathrm{a}},
$$

obey

$$
\left\{\Gamma^{\mu}, \Gamma^{\nu}\right\}=2 g^{\mu \nu}, \quad\left[\Sigma_{\mathrm{a}}, \Sigma_{\mathrm{b}}\right]=i \epsilon_{\mathrm{abc}} \Sigma_{\mathrm{c}}, \quad\left[\Gamma^{\mu}, \Sigma_{\mathrm{a}}\right]=0,
$$

for $\mu, \nu=0,1,2$ and $a, b, c=1,2,3$ and we have regrouped the gauge fields into

$$
B_{\mu} \equiv b_{\mu}^{0}+b_{\mu}^{\mathrm{a}} \Sigma^{\mathrm{a}},
$$

following the prescription,

$$
b_{\mu}^{0}:=-a_{\mu}, \quad b_{\mu}^{1}:=b_{\mu}^{2}:=0, \quad b_{\mu}^{3}:=+a_{5 \mu} .
$$

Notice that

$$
\Sigma_{3}=-\gamma_{5}
$$

so that the symmetry under the $\mathrm{U}(1) \otimes \mathrm{U}(1)$ local gauge transformation (3.2) has become the invariance of the Lagrangian in Eq. (3.4a), (3.4b), (3.4c), (3.4d), and (3.4e) under

$$
\begin{gathered}
\bar{\chi} \rightarrow \bar{\chi} e^{-i\left(\phi-\phi_{5} \Sigma_{3}\right)}, \quad \chi \rightarrow e^{+i\left(\phi-\phi_{5} \Sigma_{3}\right)} \chi, \\
b_{\mu}^{0} \rightarrow b_{\mu}^{0}+\partial_{\mu} \phi, \quad b_{\mu}^{3} \rightarrow b_{\mu}^{3}+\partial_{\mu} \phi_{5}, \\
b_{\mu}^{1} \rightarrow b_{\mu}^{1}, \quad b_{\mu}^{2} \rightarrow b_{\mu}^{2}, \quad \theta \rightarrow \theta-2 \phi_{5} .
\end{gathered}
$$

\section{Hidden U(2) non-Abelian structure}

To make the $\mathrm{U}(2)$ non-Abelian structure explicit, observe first that the mass $m n_{\mathrm{a}} \Sigma_{\mathrm{a}}$ is an element of an su(2) Lie algebra. Indeed, there exists a $4 \times 4$ matrix $U$ representing an 
element of $\mathrm{SU}(2)$ generated by $\Sigma_{\mathrm{a}} \mathrm{a}=1,2,3$ such that

$$
m n_{\mathrm{a}} \Sigma_{\mathrm{a}}=m U \Sigma_{3} U^{\dagger} \text {. }
$$

We then infer that the partition functions (3.1a), (3.1b), (3.1c), and (3.1d) or, equivalently, (3.4a), (3.4b), (3.4c), $(3.4 \mathrm{~d})$, and $(3.4 \mathrm{e})$ are special cases of the more general partition function,

$$
\begin{gathered}
Z:=\int \mathcal{D}[\bar{\chi}, \chi] \exp \left(i \int d^{3} x \mathcal{L}_{m, \eta}\right), \\
\mathcal{L}_{m, \eta}:=\bar{\chi}\left(\Gamma^{\mu} i \partial_{\mu}+\Gamma^{\mu} B_{\mu}-m U \Sigma_{3} U^{\dagger}-\eta\right) \chi,
\end{gathered}
$$

where

$$
B_{\mu}(x)=b_{\mu}^{0}(x)+b_{\mu}^{\mathrm{a}}(x) \Sigma^{\mathrm{a}}, \quad \mu=0,1,2,
$$

are arbitrary elements of the Lie algebra $\mathrm{u}(2)=\mathrm{u}(1) \oplus \mathrm{su}(2)$ and

$$
U(x)=e^{i u_{0}(x)} e^{i u_{\mathrm{a}}(x) \Sigma_{\mathrm{a}}}, \quad u_{0}(x), u_{\mathrm{a}}(x) \in \mathrm{R}
$$

is an arbitrary element of $\mathrm{U}(2)$. As the mapping between the unit vector $\boldsymbol{n}(x)$ and $U(x)$ is one to many, the Lagrangian and the Grassmann measure in Eq. (3.8a), (3.8b), and (3.8c) are both invariant under the local $\mathrm{U}(2)$ gauge transformation,

$$
\begin{gathered}
\bar{\chi} \rightarrow \bar{\chi} V^{\dagger}, \quad \chi \rightarrow V \chi \\
B_{\mu} \rightarrow V B_{\mu} V^{\dagger}-i V^{\dagger} \partial_{\mu} V, \\
U \rightarrow V U,
\end{gathered}
$$

parametrized by the smooth space and time dependent,

$$
V(x):=e^{i\left[v_{0}(x)+v_{\mathrm{a}}(x) \Sigma_{\mathrm{a}}\right]} \in \mathrm{U}(2),
$$

and under the global $\mathrm{U}(1) \times \mathrm{U}(1)$ transformation,

$$
U \rightarrow U W, \quad W:=e^{i \phi_{0}} e^{i \phi_{3} \Sigma_{3}},
$$

parametrized by the real-valued numbers $\phi_{0}$ and $\phi_{3}$.

Transformation (3.2) or, equivalently, Eq. (3.6) is represented by transformation (3.9a), (3.9b), and (3.9c) with $B_{\mu}$ given in Eqs. (3.4d) and (3.4e) and $U$ given by

$$
U=e^{+i \theta \Sigma_{3} / 2} e^{-i \alpha \Sigma_{2} / 2} e^{-i \theta \Sigma_{3} / 2}
$$

whereby the unit vector (3.1d) is parametrized by

$$
\boldsymbol{n}=(\sin \alpha \cos \theta,-\sin \alpha \sin \theta, \cos \alpha)^{t} .
$$

(Recall that $\cos \alpha:=\mu_{\mathrm{s}} / m, \sin \alpha:=|\Delta| / m$, and that the phase $\theta=-\arg \Delta$ is space and time dependent.)

A gradient expansion for the partition function (3.8a), (3.8b), and (3.8c) with an arbitrary space and time dependent $U \in \mathrm{SU}(2)$ but with $B_{\mu}=0$ and $\eta=0$ was performed by Jaroszewicz and shown to produce the effective action for the $\mathrm{O}(3)$ nonlinear-sigma model (NLSM) modified by a Hopf term. ${ }^{37-41}$ This Hopf term was shown by Chen and Wilczek to vanish as soon as the TRS-breaking mass $\eta$ is larger in magnitude than the TRS mass $m$. Chen and Wilczek also showed that an Abelian Chern-Simons term for a nonvanishing $b_{\mu}^{0} \equiv a_{\mu}$ is present if and only if $|\eta|>m$.

Hopf or Chern-Simons terms can cause the fractionalization of quantum numbers. Although charge fractionalization can here also be deduced from the presence of midgap single-particle states of the Dirac Hamiltonian (2.1a), (2.1b), (2.1c), (2.1d), and (2.1e) in static backgrounds, ${ }^{10,15,16,19,37}$ it is natural to explore the emergence of fractional statistics under the exchange of pointlike quasiparticles by exploring the fully dynamical theory encoded by the partition function $(3.4 \mathrm{a}),(3.4 \mathrm{~b}),(3.4 \mathrm{c}),(3.4 \mathrm{~d})$, and $(3.4 \mathrm{e})$. To this end, it is essential to preserve all symmetries as we did up to now. The pointlike quasiparticle whose braiding statistics we shall derive are vortices ${ }^{10}$ in the dynamical phase $\theta=-\arg \Delta$, including the case when they are accompanied by axial gauge half fluxes in $a_{5 \mu}$ that screen the interactions between vortices. ${ }^{19}$

\section{DERIVATIVE EXPANSION AND THE EFFECTIVE ACTION}

It is known that the Dirac Hamiltonian (2.1a), (2.1b), (2.1c), (2.1d), and (2.1e) with static backgrounds can support zero modes. ${ }^{10,15,16,19,37}$ This can be of a nuisance when computing a fermion determinant. However, it is possible to elegantly dispose of this difficulty with the help of the observation made by Jaroszewicz that a nonsingular U(2) gauge transformation on the Dirac Kernel in the partition function (3.8a), (3.8b), and (3.8c) can turn a single-particle midgap state into a single-particle threshold state without changing the spectral asymmetry. ${ }^{37,42}$ This is achieved by redefining the Grassmann integration variables in the partition function (3.8a), (3.8b), and (3.8c) according to

$$
\bar{\chi}=: \bar{\chi}^{\prime} U^{\dagger}, \quad \chi=: U \chi^{\prime} \text {. }
$$

The partition function $(3.8 \mathrm{a}),(3.8 \mathrm{~b})$, and $(3.8 \mathrm{c})$ becomes

$$
\begin{gathered}
Z_{m, \eta}^{\prime}\left[B_{\mu}^{\prime}\right]:=\int \mathcal{D}\left[\bar{\chi}^{\prime}, \chi^{\prime}\right] \exp \left(i \int d^{3} x \mathcal{L}_{m, \eta}^{\prime}\right), \\
\mathcal{L}_{m, \eta}^{\prime}:=\bar{\chi}^{\prime}\left(\Gamma^{\mu} i \partial_{\mu}+\Gamma^{\mu} B_{\mu}^{\prime}-m \Sigma_{3}-\eta\right) \chi^{\prime},
\end{gathered}
$$

where

$$
B_{\mu}^{\prime}=U^{\dagger} B_{\mu} U+U^{\dagger} i \partial_{\mu} U
$$

need not be a pure gauge because of the term $U^{\dagger} B_{\mu} U$.

Symmetries (3.9a), (3.9b), and (3.9c) of the Lagrangian and the Grassmann measure in Eq. (3.8a), (3.8b), and (3.8c) become the invariance of the Lagrangian and the Grassmann measure in Eq. (4.2a) and (4.2b) under the local U(2) gauge symmetry,

$$
\begin{gathered}
\bar{\chi}^{\prime} \rightarrow \bar{\chi}^{\prime}, \quad \chi^{\prime} \rightarrow \chi^{\prime}, \\
B_{\mu} \rightarrow V B_{\mu} V^{\dagger}-i V^{\dagger} \partial_{\mu} V, \\
U \rightarrow V U,
\end{gathered}
$$

parametrized by the space- and time-dependent $V(x) \in \mathrm{U}(2)$ and under the global $\mathrm{U}(1) \times \mathrm{U}(1)$ gauge symmetry,

$$
\bar{\chi}^{\prime} \rightarrow \bar{\chi}^{\prime} W, \quad \chi^{\prime} \rightarrow W^{\dagger} \chi^{\prime}, \quad U \rightarrow U W,
$$

parametrized by the space and time independent $W$ $:=\exp \left(i \phi_{0}\right) \exp \left(i \phi_{3} \Sigma_{3}\right)$. Notice that 


$$
B_{\mu}^{\prime} \rightarrow W^{\dagger} B_{\mu}^{\prime} W
$$

under transformation (4.3a) and (4.3b).

Evidently, the transformed Dirac fermions are local U(2) gauge singlets. Thus, by dressing the original Dirac fermions into local $\mathrm{U}(2)$ gauge singlets, any midgap single-particle states from the original static Dirac Hamiltonian has migrated to the threshold of the continuum part of the transformed single-particle spectrum, provided the single-particle spectral gap has not closed, i.e., $m \neq|\eta|$ in the parameter space $(m, \eta) \in \mathbb{R}^{2}$ of Fig. 1 . This dressing is achieved without changing the spectral asymmetry in any region of Fig. 1 in which the single-particle gap remains open for the $\mathrm{U}(2)$ gauge transformation is not singular.

The parametrization

$$
\begin{gathered}
b_{\mu}^{\prime 0}=-a_{\mu}, \\
b_{\mu}^{\prime 1}=-\sin \alpha \cos \theta\left(a_{5 \mu}-\frac{1}{2} \partial_{\mu} \theta\right), \\
b_{\mu}^{\prime 2}=+\sin \alpha \sin \theta\left(a_{5 \mu}-\frac{1}{2} \partial_{\mu} \theta\right), \\
b_{\mu}^{\prime 3}=+\left(\cos \alpha a_{5 \mu}+\frac{1-\cos \alpha}{2} \partial_{\mu} \theta\right),
\end{gathered}
$$

of $B_{\mu}^{\prime}=b_{\mu}^{\prime 0}+b_{\mu}^{\prime a} \Sigma^{\mathrm{a}}$ where $\mu=0,1,2$ follows from inserting Eqs. (3.4d), (3.4e), and (3.10), into Eq. (4.2b). The transformation law of Eq. (4.5) under the local $\mathrm{U}(1) \otimes \mathrm{U}(1)$ gauge transformation (3.2) is

$$
\begin{gathered}
b_{\mu}^{\prime 0} \rightarrow b_{\mu}^{\prime 0}+\partial_{\mu} \phi, \\
b_{\mu}^{\prime 1} \rightarrow \cos \left(2 \phi_{5}\right) b_{\mu}^{\prime 1}-\sin \left(2 \phi_{5}\right) b_{\mu}^{\prime 2}, \\
b_{\mu}^{\prime 2} \rightarrow \sin \left(2 \phi_{5}\right) b_{\mu}^{\prime 1}+\cos \left(2 \phi_{5}\right) b_{\mu}^{\prime 2}, \\
b_{\mu}^{\prime 3} \rightarrow b_{\mu}^{\prime 3}-\partial_{\mu} \phi_{5} .
\end{gathered}
$$

At this stage, it is convenient to define the effective action (Lagrangian)

$$
S_{m, \eta}^{\mathrm{eff}}\left[B_{\mu}^{\prime}\right] \equiv \int d^{3} x \mathcal{L}_{m, \eta}^{\mathrm{eff}}:=-i \ln Z_{m, \eta}^{\prime}\left[B_{\mu}^{\prime}\right]
$$

in the background field $B_{\mu}^{\prime}$ given by Eq. (4.6). This effective action is constrained by the gauge symmetries in the following way.

Any transformation of the Grassmann integration variables $\bar{\chi}^{\prime}$ and $\chi^{\prime}$ with unity for the Jacobian leaves the numerical value of the partition function (4.2a) and (4.2b) unchanged. As the Grassmann measure in the partition function (4.2a) and (4.2b) is invariant under the local $\mathrm{U}(1) \otimes \mathrm{U}(1)$ transformation,

$$
\bar{\chi}^{\prime} \rightarrow \bar{\chi}^{\prime} V^{\dagger}, \quad \chi^{\prime} \rightarrow V \chi^{\prime}, \quad V:=e^{+i\left(\phi-\phi_{5} \Sigma_{3}\right)},
$$

it follows that
TABLE I. Coefficients for the effective action in Eq. (4.10a) and (4.10b). The calculation leading to these values is presented in Appendix A.

\begin{tabular}{lcccc}
\hline \hline & $C_{11}^{(0)}$ & $C_{00}^{(1)}=C_{33}^{(1)}$ & $C_{11}^{(1)}$ & $C_{03}^{(1)}$ \\
\hline$|\eta|<m$ & $\frac{3 m^{2}-\eta^{2}}{6 \pi m}$ & 0 & $\frac{\eta}{6 \pi m}$ & $\frac{1}{2 \pi} \operatorname{sgn} \mu_{\mathrm{s}}$ \\
$m<|\eta|$ & $\frac{m^{2}}{3 \pi|\eta|}$ & $\frac{1}{4 \pi} \operatorname{sgn} \eta$ & $\frac{3 \eta^{2}-m^{2}}{12 \pi \eta^{2}} \operatorname{sgn} \eta$ & 0 \\
\hline \hline
\end{tabular}

$$
Z_{m, \eta}^{\prime}\left[B_{\mu}^{\prime}\right]=Z_{m, \eta}^{\prime}\left[V^{\dagger} B_{\mu}^{\prime} V-V^{\dagger} i \partial_{\mu} V\right] .
$$

The partition function $(4.2 \mathrm{a})$ and $(4.2 \mathrm{~b})$ thus takes the form

$$
Z_{m, \eta}^{\prime}\left[B_{\mu}^{\prime}\right]=\exp \left(i \int d^{3} x \mathcal{L}_{m, \eta}^{\mathrm{eff}}\right)
$$

where

$$
\begin{aligned}
\mathcal{L}_{m, \eta}^{\mathrm{eff}}= & C_{11}^{(0)}\left(b^{\prime 1 \rho} b_{\rho}^{\prime 1}+b^{\prime 2 \rho} b_{\rho}^{\prime 2}\right)+C_{00}^{(1)} \epsilon^{\nu \rho \kappa} b_{\nu}^{\prime 0} \partial_{\rho} b_{\kappa}^{\prime 0} \\
& +C_{33}^{(1)} \epsilon^{\nu \rho \kappa} b_{\nu}^{\prime 3} \partial_{\rho} b_{\kappa}^{\prime 3}+C_{11}^{(1)} \epsilon^{\nu \rho \kappa}\left(b_{\nu}^{\prime 1} \partial_{\rho} b_{\kappa}^{\prime 1}+b_{\nu}^{\prime 2} \partial_{\rho} b_{\kappa}^{\prime 2}\right. \\
& \left.-2 \epsilon^{\mathrm{ab} 3} b_{\nu}^{\prime \mathrm{a}} b_{\rho}^{\prime \mathrm{b}} b_{\kappa}^{\prime 3}\right)+C_{03}^{(1)} \epsilon^{\nu \rho \kappa} b_{\nu}^{\prime 0} \partial_{\rho} b_{\kappa}^{\prime 3}+\cdots, \quad(4.10 \mathrm{~b})
\end{aligned}
$$

up to first order in a derivative expansion. This Lagrangian changes by the usual Abelian Chern-Simons boundary terms under gauge transformation (4.6). The real-valued coefficients $C_{11}^{(0)}, C_{00}^{(1)}, C_{33}^{(1)}, C_{11}^{(1)}$, and $C_{03}^{(1)}$ are functions of the parameters $m \in \mathbb{R}$ and $\eta \in \mathbb{R}$ with $m \neq|\eta|$. A tedious calculation, summarized in Appendix A, yields the values shown in Table I.

Observe that the coefficients $C_{00}^{(1)}, C_{33}^{(1)}$, and $C_{03}^{(1)}$ that multiply the terms fixed by the local $\mathrm{U}(1) \otimes \mathrm{U}(1)$ gauge invariance in the effective Lagrangian (4.10b) can only take a discrete set of values, while the coefficients $C_{11}^{(0)}$ and $C_{11}^{(1)}$ that multiply the terms fixed by the global $U(1)$ gauge invariance can vary continuously with $m$ and $\eta$.

The case $m=0$ when TRS is maximally broken is special as the symmetry-breaking term $m \Sigma_{3}$ drops out from the Lagrangian in Eq. (4.2a) and (4.2b). The matrix $V$ in the change in Grassmann variables (4.8) is then not restricted to the Abelian subgroup $\mathrm{U}(1) \otimes \mathrm{U}(1)$ of $\mathrm{U}(1) \otimes \mathrm{SU}(2)$ but can be arbitrarily chosen in $\mathrm{U}(2)$. Consequently, $C_{33}^{(1)}=C_{11}^{(1)}$ in this limit, which is consistent with the values in Table I. These (equal) coefficients then multiply an SU(2) non-Abelian Chern-Simons term when $m=0$, and hence must be quantized, ${ }^{43}$ i.e.,

$$
\begin{aligned}
\mathcal{L}_{m=0, \eta}^{\mathrm{eff}}= & \frac{\operatorname{sgn} \eta}{4 \pi} \epsilon^{\nu \rho \kappa}\left(\delta^{\mathrm{ab}} b_{\nu}^{\prime \mathrm{a}} \partial_{\rho} b_{\kappa}^{\prime \mathrm{b}}-\frac{2}{3} \epsilon^{\mathrm{abc}} b_{\nu}^{\prime \mathrm{a}} b_{\rho}^{\prime \mathrm{b}} b_{\kappa}^{\prime \mathrm{c}}\right) \\
& +\frac{\operatorname{sgn} \eta}{4 \pi} \epsilon^{\nu \rho \kappa} b_{\nu}^{\prime 0} \partial_{\rho} b_{\kappa}^{\prime 0}+\cdots
\end{aligned}
$$

where the second line on the right-hand side is nothing but the level $1 \mathrm{SU}(2)$ Chern-Simons term.

In the case $\eta=0$ when TRS holds Eq. (4.10b) simplifies to 


$$
\mathcal{L}_{m, \eta=0}^{\mathrm{eff}}=\frac{m}{2 \pi}\left(b^{\prime 1 \rho} b_{\rho}^{\prime 1}+b^{\prime 2 \rho} b_{\rho}^{\prime 2}\right)+\frac{\operatorname{sgn} \mu_{\mathrm{s}}}{2 \pi} \epsilon^{\nu \rho \kappa} b_{\nu}^{\prime 0} \partial_{\rho} b_{\kappa}^{\prime 3}+\cdots
$$

Notice that the second line is a double Chern-Simons term on the fields $b^{\prime 0}$ and $b^{\prime 3}$ which is also called a BF ChernSimons theory. ${ }^{44,45}$

We close this section with the main intermediary step of this paper from which the fractionalization of the fermion charge and statistical phase follows. Insertion of Eq. (4.5) into Eq. (4.10a) and (4.10b) gives the effective action,

$$
\begin{aligned}
\mathcal{L}_{m, \eta}^{\mathrm{eff}}= & C_{11}^{(0)} \sin ^{2} \alpha\left(a_{5}^{\rho}-\frac{1}{2} \partial^{\rho} \theta\right)\left(a_{5 \rho}-\frac{1}{2} \partial_{\rho} \theta\right) \\
& +C_{00}^{(1)} \epsilon^{\nu \rho \kappa} a_{\nu} \partial_{\rho} a_{\kappa}+C_{33}^{(1)} \epsilon^{\nu \rho \kappa}\left(\cos \alpha a_{5 \nu}\right. \\
& \left.+\frac{1-\cos \alpha}{2} \partial_{\nu} \theta\right) \partial_{\rho}\left(\cos \alpha a_{5 \kappa}+\frac{1-\cos \alpha}{2} \partial_{\kappa} \theta\right) \\
& +C_{11}^{(1)} \sin ^{2} \alpha \epsilon^{\nu \rho \kappa}\left(a_{5 \nu}-\frac{1}{2} \partial_{\nu} \theta\right) \partial_{\rho}\left(a_{5 \kappa}-\frac{1}{2} \partial_{\kappa} \theta\right) \\
& -C_{03}^{(1)} \epsilon^{\nu \rho \kappa} a_{\nu} \partial_{\rho}\left(\cos \alpha a_{5 \kappa}+\frac{1-\cos \alpha}{2} \partial_{\kappa} \theta\right)+\cdots
\end{aligned}
$$

with the local $\mathrm{U}(1) \otimes \mathrm{U}(1)$ gauge invariance,

$$
\begin{gathered}
a_{\mu} \rightarrow a_{\mu}-\partial_{\mu} \phi, \\
a_{5 \mu} \rightarrow a_{5 \mu}-\partial_{\mu} \phi_{5}, \quad \theta \rightarrow \theta-2 \phi_{5},
\end{gathered}
$$

for any compact and boundary-less manifold in $(2+1)$-dimensional space and time.

Some comments are of order here. First, the coefficient $C_{11}^{(0)}$ controls the axial phase stiffness of the Anderson-Higgs contribution to the effective action. Second, each of the coefficients $C_{00}^{(1)}, C_{33}^{(1)}$, and $C_{11}^{(1)}$ multiplies a Chern-Simons term that is diagonal with respect to the gauge fields. The coefficient $C_{03}^{(1)}$ is different in that regard since it couples the gauge field $a_{\mu}$ responsible for the conservation of the fermion number to the axial gauge field $a_{5 \mu}$ on the one hand, and the axial singlet linear combination $\widetilde{a}_{5 \mu} \equiv a_{5 \mu}-\partial_{\mu} \theta / 2$ on the other hand. Such an off-diagonal coupling is reminiscent of socalled BF Chern-Simons theories. ${ }^{44,45}$ It is the coefficient $C_{03}^{(1)}$ that controls the charge assignments in the field theory (4.13) and, for later convenience, we break its contribution to the induced fermionic charge into two pieces,

$$
\begin{gathered}
\mathcal{L}_{\mathrm{BF}}:=\mathcal{L}_{\mathrm{BF}}^{(1)}+\mathcal{L}_{\mathrm{BF}}^{(2)}, \\
\mathcal{L}_{\mathrm{BF}}^{(1)}:=C_{03}^{(1)}(1-\cos \alpha) a d \widetilde{a}_{5}, \\
\mathcal{L}_{\mathrm{BF}}^{(2)}:=-C_{03}^{(1)} a d a_{5} .
\end{gathered}
$$

Here, we have introduced the short-hand notation $a d b \equiv \epsilon^{\mu \nu \rho} a_{\mu} \partial_{\nu} b_{\rho}$.

\section{FRACTIONAL FERMION CHARGE}

Equipped with Eq. (4.13) and Table I we compute in this section the leading contributions in the gradient expansion to the expectation value of the conserved charge current,

$$
\left\langle j^{\mu}\right\rangle_{m, \eta}:=-\left.i \frac{\delta \ln Z_{m, \eta}^{\prime}\left[B^{\prime}\right]}{\delta a_{\mu}}\right|_{a_{\mu}=0} .
$$

The induced fermion charge current is

$$
j^{\mu}=-C_{03}^{(1)} \epsilon^{\mu \rho \kappa} \partial_{\rho}\left(\cos \alpha a_{5 \kappa}+\frac{1-\cos \alpha}{2} \partial_{\kappa} \theta\right)+\cdots .
$$

It obeys the continuity equation

$$
\partial_{\mu} j^{\mu}=0 \text {. }
$$

The total induced fermionic charge

$$
Q:=\int d^{2} \boldsymbol{r} j^{0}(\boldsymbol{r}, t)
$$

is thus time independent and given by

$$
Q=-C_{03}^{(1)} \oint d \boldsymbol{l} \cdot\left(\cos \alpha \boldsymbol{a}_{5}+\frac{1-\cos \alpha}{2} \boldsymbol{\partial} \theta\right),
$$

with the help of Stokes' theorem. The induced fermionic charge is

$$
Q=-2 \pi C_{03}^{(1)}\left(\frac{n_{\theta}}{2}+\frac{1}{2}\left(n_{5}-n_{\theta}\right) \cos \alpha\right)
$$

for the special case when the vector fields $\boldsymbol{a}_{5}$ and $\boldsymbol{\partial} \theta$ support, on a circular boundary at infinity, the net vorticity

$$
\begin{aligned}
& a_{5}^{i} \rightarrow-\frac{n_{5}}{2} \epsilon^{i j} \frac{r^{j}}{\boldsymbol{r}^{2}}, \quad n_{5} \in \mathbb{Z}, \\
& \partial^{i} \theta \rightarrow-n_{\theta} \epsilon^{i j} \frac{r^{j}}{\boldsymbol{r}^{2}}, \quad n_{\theta} \in \mathbb{Z},
\end{aligned}
$$

respectively. In the absence of the axial gauge flux $n_{5}=0$, while the condition for the axial vorticity to screen the (Kekulé) vorticity is $n_{5}=n_{\theta}$. Notice that because $C_{03}^{(1)}$ vanishes for $|\eta|>m$, there is no charge bound to the vortices in that regime. In contrast, when $|\eta|<m$, the charge bound to the topological defect is

$$
Q=-\operatorname{sgn} \mu_{\mathrm{s}} \times \begin{cases}\sin ^{2} \frac{\alpha}{2} n_{\theta}, & \text { unscreened }\left(n_{5}=0\right) \\ \frac{1}{2} n_{\theta}, & \text { screened }\left(n_{5}=n_{\theta}\right) .\end{cases}
$$

These results are consistent with those in Refs. 10, 15, and 16. 


\section{FRACTIONAL STATISTICAL ANGLE}

We start from the effective partition function

$$
Z_{m, \eta}^{\mathrm{eff}}:=\int \mathcal{D}\left[a^{\mu}, a_{5}^{\mu}, \theta\right] \exp \left(i \int d^{3} x \mathcal{L}_{m, \eta}^{\mathrm{eff}}\right),
$$

with the Lagrangian given by Eq. (4.13) and the coefficients in Table I. In a static approximation, i.e., if we ignore dynamics as we did when computing the fractional charge (5.7), vortices are independently supported by the axial gauge field $a_{5}^{\mu}$ or by the phase $\theta$.

We will analyze the exchange statistics in two separate cases. The first is when the $\theta$ vortices are dynamically screened by the half fluxes in the axial gauge field $a_{5}^{\mu}$. The second case is when the axial gauge field is suppressed, and the $\theta$ vortex is unscreened; this situation does not arise from the effective Lagrangian (4.13) itself, but it can occur when one goes beyond the linearized Dirac approximation or includes other lattice effects.

\section{A. Screened vortices}

The exchange statistics of vortices and axial gauge fluxes follows from the effective Lagrangian for the so-called vortex currents. One way to obtain this effective Lagrangian in the screened case is to notice that the local axial gauge invariance together with the first line in Eq. (4.13) provides the screening condition for the axial gauge potential must then track the $\theta$ field and, in particular, vortices in $\theta$ must be screened by half fluxes in $a_{5}^{\mu}$.

One way to impose this screening is to replace

$$
\partial_{\mu} \theta-2 a_{5 \mu} \rightarrow 0
$$

in Eq. (4.13). This can be justified more precisely by using the (vortex) dual description of the $X Y$ model, as presented in Appendix B. In effect, the fluctuations away from condition (6.2), which are penalized by the finite stiffness coefficient $C_{11}^{(0)} \sin ^{2} \alpha$, can be accounted through a Maxwell term in the dual description. However, the Maxwell term does not enter the exchange statistics. Thus, we can simply use the infinite stiffness limit or, equivalently, condition (6.2).

The Lagrangian given by Eq. (4.13) in the screening limit (6.2) is

$$
\begin{aligned}
\mathcal{L}_{m, \eta}^{\mathrm{eff}}= & C_{00}^{(1)} \epsilon^{\nu \rho \kappa} a_{\nu} \partial_{\rho} a_{\kappa}+\frac{1}{4} C_{33}^{(1)} \epsilon^{\nu \rho \kappa}\left(\partial_{\nu} \theta\right) \partial_{\rho}\left(\partial_{\kappa} \theta\right) \\
& -\frac{1}{2} C_{03}^{(1)} \epsilon^{\nu \rho \kappa} a_{\nu} \partial_{\rho}\left(\partial_{\kappa} \theta\right)+\cdots
\end{aligned}
$$

The Lagrangian can be written in terms of the vortex current,

$$
\bar{j}_{\mathrm{vrt}}^{\mu}:=\frac{1}{2 \pi} \epsilon^{\mu \nu \lambda} \partial_{\nu} \partial_{\lambda} \theta
$$

that obeys the conservation law,

$$
\partial_{\mu} \bar{j}_{\mathrm{vrt}}^{\mu}=0,
$$

using the duality representation of the $X Y$ model supplemented by a Chern-Simons term in $(2+1)$ space and time as done in Appendix B. This leads to the Chern-Simons Lagrangian,

$$
\begin{aligned}
\mathcal{L}_{m, \eta}^{\mathrm{eff}}= & C_{00}^{(1)} \epsilon^{\nu \rho \kappa} a_{\nu} \partial_{\rho} a_{\kappa}-\pi C_{03}^{(1)} a_{\nu} \bar{j}_{\mathrm{vrt}}^{\nu} \\
& +\frac{1}{4} C_{33}^{(1)}\left(\epsilon^{\nu \rho \kappa} d_{\nu} \partial_{\rho} d_{\kappa}+4 \pi d_{\nu} \bar{j}_{\mathrm{vrt}}^{\nu}\right)+\cdots,
\end{aligned}
$$

from which the statistics carried by screened quasiparticles with the current $\bar{j}_{\mathrm{vrt}}^{v}$ follows. This statistics depends on the coefficients in Table I. We treat separately the two phases of Fig. 1.

\section{Weak time-reversal symmetry breaking: $|\eta|<m$}

In this limit, $C_{00}^{(1)}=C_{33}^{(1)}=0$ and the effective Lagrangian (6.6) reduces to

$$
\mathcal{L}_{m, \eta}^{\mathrm{eff}}=-\frac{1}{2} \operatorname{sgn} \mu_{\mathrm{s}} a_{\nu} \bar{j}_{\mathrm{vrt}}^{\nu}+\cdots .
$$

Thus, because of the absence of the Chern-Simons terms, the statistical angle $\Theta$ under exchange of any two screened quasiparticles is bosonic,

$$
\frac{\Theta}{\pi}=0
$$

Notice that it also follows that the induced fermionic U(1) current,

$$
j^{\nu}=-\frac{1}{2} \operatorname{sgn} \mu_{\mathrm{s}} \bar{j}_{\mathrm{vrt}}^{\nu},
$$

that couples linearly to $a_{\mu}$, is tied to the vortex current. In other words, screened quasiparticles with unit vorticity are charged objects with charge $Q= \pm 1 / 2$ as found in Refs. 10, 15, and 16 and in Sec. V.

\section{Strong time-reversal symmetry breaking: $|\eta|>m$}

In this limit, $C_{00}^{(1)}=C_{33}^{(1)}=(4 \pi)^{-1} \operatorname{sgn} \eta$ and the effective Lagrangian (6.6) reduces to

$$
\begin{aligned}
\mathcal{L}_{m, \eta}^{\text {eff }}= & \frac{1}{4 \pi} \operatorname{sgn} \eta \epsilon^{\nu \rho \kappa} a_{\nu} \partial_{\rho} a_{\kappa} \\
& +\frac{1}{16 \pi} \operatorname{sgn} \eta\left(\epsilon^{\nu \rho \kappa} d_{\nu} \partial_{\rho} d_{\kappa}+4 \pi d_{\nu} \bar{j}_{\mathrm{vrt}}^{\nu}\right)+\cdots .
\end{aligned}
$$

Using the coefficient of the Chern-Simons Lagrangian for the gauge field $d_{\nu}$ and its coupling to the vortex current $\bar{j}_{\mathrm{vrt}}^{\nu}$ (see Appendix B for the relation between the statistical angle and the coefficient in front of the Chern-Simons term), the statistical angle $\Theta$ under exchange of two screened quasiparticles with unit vorticity is

$$
\frac{\Theta}{\pi}=\frac{1}{4} \operatorname{sgn} \eta .
$$

Notice that the U(1) current now vanishes, i.e., screened quasiparticles carrying fractional statistics are now charge neutral. 


\section{B. Unscreened vortices}

We turn to the situation when the axial gauge half fluxes are suppressed, while $\theta$ vortices are still present. We call these vortices unscreened quasiparticles. This situation arises if, in addition to the effective Lagrangian (4.13) which followed from integrating out the Dirac fermions, there are terms in the effective Lagrangian due to lattice degrees of freedom that break the axial gauge symmetry. For instance, acoustic phonons and ripples in graphene can bring about the axial vector potential $a_{5}^{\mu}$; however, in these cases there is an energy penalty of the form $a_{5 \mu} a_{5}^{\mu}$ that breaks the axial gauge invariance due to contributions to the elastic energy.

The case when the axial gauge potential is absent, i.e., the quasiparticles are unscreened, is implemented by the replacement

$$
a_{5 \mu} \rightarrow 0
$$

in Eq. (4.13). There follows

$$
\begin{aligned}
\mathcal{L}_{m, \eta}^{\mathrm{eff}}= & \frac{1}{4} C_{11}^{(0)} \sin ^{2} \alpha\left(\partial^{\rho} \theta\right)\left(\partial_{\rho} \theta\right) \\
& +\left(C_{33}^{(1)} \sin ^{2} \frac{\alpha}{2}+C_{11}^{(1)} \cos ^{2} \frac{\alpha}{2}\right) \sin ^{2} \frac{\alpha}{2} \epsilon^{\nu \rho \kappa}\left(\partial_{\nu} \theta\right) \partial_{\rho}\left(\partial_{\kappa} \theta\right) \\
& +C_{00}^{(1)} \epsilon^{\nu \rho \kappa} a_{\nu} \partial_{\rho} a_{\kappa}-C_{03}^{(1)} \sin ^{2} \frac{\alpha}{2} \epsilon^{\nu \rho \kappa} a_{\nu} \partial_{\rho}\left(\partial_{\kappa} \theta\right)+\cdots
\end{aligned}
$$

This Lagrangian can be dualized with the help of the vortex current (6.4) (see Appendix B),

$$
\begin{aligned}
\mathcal{L}_{m, \eta}^{\text {eff }}= & -\left(8 \pi^{2} C_{11}^{(0)} \sin ^{2} \alpha\right)^{-1} f^{\mu \nu} f_{\mu \nu}+c_{\mu} \bar{j}_{\mathrm{vrt}}^{\mu}+C_{00}^{(1)} \epsilon^{\nu \rho \kappa} a_{\nu} \partial_{\rho} a_{\kappa} \\
& -2 \pi C_{03}^{(1)} \sin ^{2} \frac{\alpha}{2} a_{\nu} \bar{j}_{\mathrm{vrt}}^{\nu} \\
& +\left(C_{33}^{(1)} \sin ^{2} \frac{\alpha}{2}+C_{11}^{(1)} \cos ^{2} \frac{\alpha}{2}\right) \\
& \times \sin ^{2} \frac{\alpha}{2}\left(\epsilon^{\nu \rho \kappa} d_{\nu} \partial_{\rho} d_{\kappa}+4 \pi d_{\nu} \bar{j}_{\mathrm{vrt}}^{\nu}\right)+\cdots
\end{aligned}
$$

(The Maxwell term $f_{\mu \nu} f^{\mu \nu}$ is associated to the gauge potential $c_{\mu}$, see Appendix B.) We shall denote with $\mathcal{L}_{c}$ the first line of Eq. (6.14). The statistics carried by unscreened quasiparticles with the current $j_{\text {vrt }}^{\mu}$ follows. This statistics depends on the coefficients in Table I. We treat separately the two phases of Fig. 1.

\section{Weak time-reversal symmetry breaking: $|\eta|<m$}

In this limit, $\quad C_{00}^{(1)}=C_{33}^{(1)}=0, \quad C_{11}^{(1)}=\eta /(6 \pi m), \quad C_{03}^{(1)}$ $=(2 \pi)^{-1} \operatorname{sgn} \mu_{s}$, and the effective Lagrangian (6.14) reduces to

$$
\begin{aligned}
\mathcal{L}_{m, \eta}^{\mathrm{eff}}= & \mathcal{L}_{c}-\operatorname{sgn} \mu_{\mathrm{s}} \sin ^{2} \frac{\alpha}{2} a_{\nu} \bar{j}_{\mathrm{vrt}}^{\nu} \\
& +\frac{\eta}{24 \pi m} \sin ^{2} \alpha\left(\epsilon^{\nu \rho \kappa} d_{\nu} \partial_{\rho} d_{\kappa}+4 \pi d_{\nu} \bar{j}_{\mathrm{vrt}}^{\nu}\right) .
\end{aligned}
$$

Using the coefficient of the Chern-Simons Lagrangian for the gauge field $d_{\nu}$ and its coupling to the vortex current $\bar{j}_{\mathrm{vrt}}^{\nu}$ (see Appendix B for the relation between the statistical angle and the coefficient in front of the Chern-Simons term), the statistical angle $\Theta$ under exchange of two unscreened quasiparticles with unit vorticity is

$$
\frac{\Theta}{\pi}=\frac{\eta}{6 m} \sin ^{2} \alpha=\frac{2 \eta}{3 m}|Q|(1-|Q|)
$$

by Eq. (5.7). Notice that it also follows that the induced fermionic $\mathrm{U}(1)$ current,

$$
j^{\nu}=-\operatorname{sgn} \mu_{\mathrm{s}} \sin ^{2} \frac{\alpha}{2} \bar{j}_{\mathrm{vrt}}^{\nu}
$$

that couples linearly to $a_{\mu}$, is tied up to the vortex current. In other words, unscreened quasiparticles with unit vorticity are charged objects with charge $Q= \pm \sin ^{2}(\alpha / 2)$ that varies continuously as a function of the ratio $\mu_{\mathrm{s}} / m$ [see Eq. (5.7)] as found in Refs. 10, 15, and 16.

\section{Strong time-reversal symmetry breaking: $|\eta|>m$}

In this limit, $\quad C_{00}^{(1)}=C_{33}^{(1)}=(4 \pi)^{-1} \operatorname{sgn} \eta, \quad C_{11}^{(1)}$ $=\left(3 \eta^{2}-m^{2}\right) /\left(12 \pi \eta^{2}\right) \operatorname{sgn} \eta, C_{03}^{(1)}=0$, and the effective Lagrangian (6.14) reduces to

$$
\begin{aligned}
\mathcal{L}_{m, \eta}^{\mathrm{eff}}= & \mathcal{L}_{c}+\frac{1}{4 \pi} \operatorname{sgn} \eta \epsilon^{\nu \rho \kappa} a_{\nu} \partial_{\rho} a_{\kappa} \\
& +\frac{1}{4 \pi} \operatorname{sgn} \eta\left[\left(1-\frac{m^{2}}{3 \eta^{2}}\right)+\frac{m^{2}}{3 \eta^{2}} \sin ^{2} \frac{\alpha}{2}\right] \\
& \times \sin ^{2} \frac{\alpha}{2}\left(\epsilon^{\nu \rho \kappa} d_{\nu} \partial_{\rho} d_{\kappa}+4 \pi d_{\nu} \bar{j}_{\mathrm{vrt}}^{\nu}\right)+\cdots .
\end{aligned}
$$

Using the coefficient of the Chern-Simons Lagrangian for the gauge field $d_{\nu}$ and its coupling to the vortex current $\bar{j}_{\mathrm{vrt}}^{\nu}$ (see Appendix B for the relation between the statistical angle and the coefficient in front of the Chern-Simons term), the statistical angle under exchange of two unscreened quasiparticles with unit vorticity is

$$
\begin{aligned}
\frac{\Theta}{\pi} & =\operatorname{sgn} \eta\left[\left(1-\frac{m^{2}}{3 \eta^{2}}\right)+\frac{m^{2}}{3 \eta^{2}} \sin ^{2} \frac{\alpha}{2}\right] \sin ^{2} \frac{\alpha}{2} \\
& =\operatorname{sgn} \eta\left[\left(1-\frac{m^{2}}{3 \eta^{2}}\right)+\frac{m^{2}}{3 \eta^{2}}|Q|\right]|Q| .
\end{aligned}
$$

Here, we have used the value of the charge $|Q|=\sin ^{2}(\alpha / 2)$ for the complementary phase $|\eta|<m$. Notice that the induced fermionic charge current $j_{\mu}$ now vanishes. The unscreened quasiparticles carrying the fractional statistics (6.19) are thus charge neutral, i.e., $|Q|$ in Eq. (6.19) should not be confused with the (now vanishing) electronic charge of unscreened quasiparticles.

We stress that the quenching of the dynamics in the axial gauge field $a_{5}^{\mu}$ implies the breaking of the axial gauge symmetry. It can be thought of as a mean-field approximation needed to interpret the numerical simulations of the Berry phase acquired by the Slater determinant of lattice fermions when one vortex is moved in a quasistatic way along a 
closed curved around another vortex. The quench approximation can also be justified if terms that break explicitly the axial gauge symmetry such as mass term for $a_{5}$ were added to Lagrangian (4.13). After all, from a microscopic point of view, axial gauge symmetry is by no means generic. The axial gauge fields can be viewed as phonon-induced fluctuations in the average separations between ions that an elastic theory generically induces. A mass term for these phonons cannot be ruled out by symmetry.

\section{Adding one more fermion to the midgap states}

All calculations for the fractional charge and exchange statistics done so far apply at zero chemical potential $\mu=0$, and at some finite staggered chemical potential $\mu_{\mathrm{s}} \neq 0$, assuming global vortex neutrality. Global vortex neutrality is imposed to bound the energy from above in the thermodynamic limit or if periodic boundary conditions are imposed. A staggered chemical potential is needed to lift the near degeneracy between the two single-particle midgap states that are exponentially localized about a vortex and antivortex in the bond-density-wave (Kekulé for graphene) order parameter $\Delta$, respectively, whose separation $r$ is much larger than $1 / \mathrm{m}$. On the one hand, when $\mu_{\mathrm{s}}=0$, the two single-particle midgap levels are, up to exponentially small corrections in $m r$, pinned to the band center $E=0$. In the thermodynamic limit, their occupancy when $\mu=0$ is then ambiguous. On the other hand, when $\mu_{\mathrm{s}} \neq 0$, the two single-particle midgap levels get pushed in opposite directions, one to $E>0$ and the other to $E<0$ (which one goes which way depends on the sign of $\mu_{\mathrm{s}}$ ). The single-particle midgap level with $E<0$ is then occupied, the other empty, when $\mu=0$ and the results of Secs. V and VI B for the fractional and exchange statistics, respectively, apply. We are going to prove that when $m>|\mu|>\left|\mu_{\mathrm{s}}\right|$ so that the two single-particle midgap levels are either both empty or both occupied, the exchange statistics is that of semions.

Suppose one adds one more electron to the Dirac sea (here defined to be the Fermi sea at $\mu=0$ ), filling the singleparticle midgap state at $E>0$. What happens to the exchange statistics?

The easiest way to answer this question is by realizing that the Berry phase accumulated by a many-body wave function that can be written as a single Slater determinant (the case in hand) is just the sum of the Berry phases for single-particle states. If we fill one more level, we only need to add the Berry phase due to that single-particle state to that of the filled Dirac sea that we already computed. The contribution from the extra level can be obtained as follows. (Here we focus on the case $\eta=0$. A generalization to $\eta \neq 0$ can be similarly formulated.)

A single-particle midgap wave function is localized near a vortex, i.e., its spatial extent is of order $1 / \mathrm{m}$. Details on $\Delta$ for distances much larger than $1 / m$ away do not matter. Hence, when winding another far-away vortex around the first one, the local order parameter $\Delta$ in the vicinity of the first vortex just sees its phase change by $2 \pi$. This allows us to focus solely on the problem of determining what happens to the single-particle midgap wave function as the phase of the or- der parameter near a vortex is rotated by $2 \pi$.

The solution for the single-particle midgap wave function when $\mu_{\mathrm{s}}=0$ and in the Dirac approximation was obtained in Ref. 10 for the unscreened vortex and in Ref. 19 for the screened vortex. In both cases, the wave function picks up a phase of $\pi$ when the phase of $\Delta$ changes by $2 \pi$. If $\mu_{\mathrm{s}} \neq 0$, the result remains the same, because while the midgap level moves with $\mu_{\mathrm{s}}$ the wave function is independent of $\mu_{\mathrm{s}}$ (the wave function has support in only one of the sublattices $\Lambda_{\mathrm{A}}$ or $\Lambda_{\mathrm{B}}$ of the underlying lattice model, so the finite value of the staggered chemical potential does not perturb the singleparticle midgap wave function).

In conclusion, occupying one additional single-particle fermion level adds a phase of $\pi$ to the many-body Berry phase when $\eta=0$. This means that the statistical angle shifts by $\Delta \Theta= \pm \pi / 2$, the statistical angle for a semion, when one fermion is added (removed) to (from) the Dirac sea.

\section{NUMERICAL CALCULATION OF THE CHARGE AND BERRY PHASE}

We are going to present numerical results on the charge and statistics of unscreened vortices supported by the bonddensity-wave (Kekulé for graphene) order parameter $\Delta$ in the presence of the compatible and competing order parameters (masses when space and time independent) $\mu_{\mathrm{s}}$ and $\eta$, respectively. The dependence of the induced fermionic charge of vortices in $\Delta$ as a function of the staggered chemical potential $\mu_{\mathrm{s}}$ was studied in Refs. 15 and 16 (see also Ref. 46 when $\left.\mu_{\mathrm{s}}=0\right)$. The following numerical results with the competing mass $\eta$ are new.

Our studies have been carried out for the honeycomb lattice, which is of direct relevance to graphene, and the square lattice with $\pi$-flux phase. Both lattice models yield consistent numerical results. In this paper, only the results for $\pi$-flux phase are presented. The relevant technical details for our numerical calculations are summarized in Appendix C. To compare the numerical with our analytical results, derived from the Dirac Hamiltonian (2.1a), (2.1b), (2.1c), (2.1d), and (2.1e), which is the continuum limit of the linearized lattice Hamiltonian, two important issues arise.

The first one is that all band curvature effects, present in any microscopic lattice model, are absent in the continuum model. Here we expect that, as long as the characteristic sizes over which the order parameters vary are large compared to the size of the unscreened vortex core, static results obtained within the continuum approximation should capture some static long-wavelength properties of the lattice model. This first expectation can be concretely addressed by the numerical studies of the induced fermionic charge of unscreened vortices below.

The second issue that arises when one starts from a lattice model is the assumed axial gauge invariance of Hamiltonian (2.1a), (2.1b), (2.1c), (2.1d), and (2.1e). This issue is subtle and substantial. Dirac Hamiltonian (2.1a), (2.1b), (2.1c), (2.1d), and (2.1e) has a local $\mathrm{U}(1) \times \mathrm{U}(1)$ gauge symmetry, while this symmetry is absent in graphene, say. Although the vector axial gauge field $\boldsymbol{a}_{5}$ is realized in graphene, say, through acoustic phonons generating ripples, and thus 
couples in an axial-gauge-invariant way to the fermions in the linear approximation, its kinetic energy is by no means required to be gauge invariant. For example, the kinetic energy of $\boldsymbol{a}_{5}$ is expected to contain the axial-gauge-symmetrybreaking mass term $\left|\boldsymbol{a}_{5}\right|^{2}$. It is thus difficult to justify the axial gauge invariance of Dirac Hamiltonian (2.1a), (2.1b), (2.1c), (2.1d), and (2.1e) in a lattice model as simple as graphene.

We do not expect predictions based on Hamiltonian (2.1a), (2.1b), (2.1c), (2.1d), and (2.1e) that rely crucially on the dynamics of the axial vector gauge field $\boldsymbol{a}_{5}$ to capture the corresponding low-energy and long-wavelength dynamical properties of graphene. We will verify this expectation with lattice computations that require the dynamics of the axial gauge field, for example, the induced Berry phase as one moves a composite particle made of a vortex and an axial gauge flux around another composite particle.

In Sec. VIII, we will present a lattice model that, by construction, has the desired local $\mathrm{U}(1) \times \mathrm{U}(1)$ gauge symmetry. This model can be used to compute numerically the statistical phases of unit bond-density-wave (Kekulé for graphene) vortices screened by axial gauge half fluxes and to verify that nonlinearities in the many-body excitation spectrum do not affect the exchange statistics of vortices separated by distances much larger than their vortex core, i.e., this is one model that regularizes Hamiltonian (2.1a), (2.1b), (2.1c), (2.1d), and (2.1e) on the lattice.

While the system presented in Sec. VIII serves by itself as a proof of principle that one can realize the local axial gauge invariance on the lattice, the computation of the exchange statistics of vortices in this lattice model is a computational challenge in lattice gauge theory, as opposed to the much simpler exercise in exact diagonalization for any noninteracting lattice model.

For this reason, we now limit the numerical studies of the statistical phases to the simpler case when $\boldsymbol{a}_{5} \rightarrow 0$, i.e., the case of unscreened vortices. In effect, we are ignoring all many-body effects imposed by the local axial gauge invariance and thus treating the problem at the mean-field level. By comparing the charge obtained from the Aharonov-Bohm effect with that obtained directly from the local density of states, we will show that this approximation is qualitatively (but not quantitatively) justified for dynamical properties of bond-density-wave (Kekulé for graphene) vortices, whereas it fails dramatically for dynamical properties of the axial gauge half fluxes.

\section{A. Static calculation of the charge}

We begin with the study of static properties, when the vortices or axial gauge half flux tubes are not moved so that the dynamics of the axial gauge potential is not relevant. One physical quantity that can be studied in the static limit is the induced fermionic fractional charge. It is obtained by summing up the local fermionic density of states in a region of space that encloses the core of the vortex.

In our numerical studies, a vortex is placed at the center of the square lattice system of size $100 \times 100$ in units of the lattice spacing $\mathfrak{a}$ while a flux of $\pi$ in units of the flux quan-

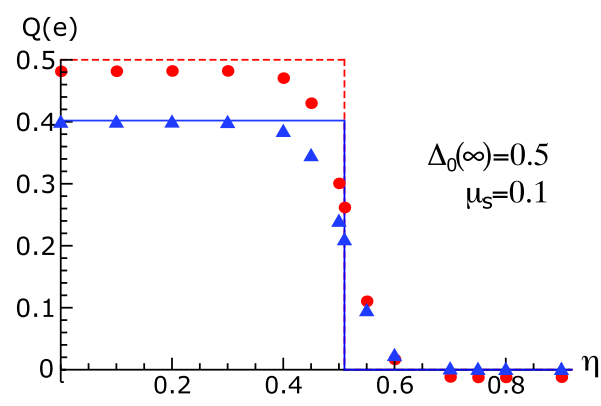

FIG. 2. (Color online) The induced fermionic charge of a quasiparticle, a unit vortex in $\Delta(\boldsymbol{r})$ with or without attachment of an axial gauge half flux in $\boldsymbol{a}_{5}(\boldsymbol{r})$, as a function of $\eta$. This charge is computed from the spectral asymmetry of spinless fermions hopping on the square lattice with lattice spacing $\mathfrak{a}$ and with a magnetic flux of $\pi$ in units of the flux quantum $\phi_{0}=h c / e$ threading each elementary plaquette in the static background of a unit vortex in $\Delta(\boldsymbol{r})$ with or without attachment of an axial gauge half flux in $\boldsymbol{a}_{5}(\boldsymbol{r})$ and for a uniform value of $\eta$. The square lattice is $100 \times 100$ in units of the lattice spacing and the area used for integrating the local density of states is a square of size $50 \times 50$ centered around a unit vortex. The following parameters were chosen: the hopping $t=1$, the magnitude of $\Delta(\boldsymbol{r})$ on the boundary is $\Delta_{0}(\infty)=0.5$, while the magnitude of the staggered chemical potential is $\mu_{\mathrm{s}}=0.1(m \approx 0.51)$. Each (red) filled circle is the induced fermionic charge of a unit vortex in $\Delta(\boldsymbol{r})$ to which an axial gauge half flux in $\boldsymbol{a}_{5}(\boldsymbol{r})$ is attached. The (red) dashed line for $\eta \leqslant m$ represents the $Q=1 / 2$ line. Each (blue) filled triangle is the induced fermionic charge of an unscreened unit vortex in $\Delta(\boldsymbol{r})$, i.e., the vector axial flux in $\boldsymbol{a}_{5}(\boldsymbol{r})$ vanishes everywhere. The (blue) solid line for $\eta \leqslant m$ represents $Q=0.402$, i.e., the predicted value from the field theory with the input parameters. When $m \gg \eta$, the induced fractional charge vanishes. A quantum phase transition at $m=\eta$, as measured by the jumped in the induced fermionic charge, is smeared by finite size effects.

tum $\phi_{0}=h c / e$ threads each elementary plaquette. An area of integration, $50 \times 50$, centered around the vortex is used for summing the local fermionic density of states. We fixed the strength of the bond-density-wave (Kekule for graphene) order parameter $\Delta=0.5$ and staggered chemical potential $\mu_{\mathrm{s}}=0.1$.

Figure 2 shows the value of the induced fermionic charge as a function of $\eta$ with and without the axial gauge half flux. A clear normalization of the fractional charge to $1 / 2$ follows from adding an axial gauge half flux. Notice that there is a (smoothed) step as the mass $\eta$ becomes comparable to $m$. This is the finite-size signature of a quantum phase transition at $|\eta|=m$. The results in Sec. V are displayed in Fig. 2. They correspond to sharp step functions at the transition point $|\eta|=m$. The numerical results displayed in Fig. 2 are consistent with the analytical results (5.7), keeping in mind that the lattices studied are finite and thus quantum transitions are smeared. For that matter, notice that the agreement between the field-theory prediction and numerics is best away from the critical point $|\eta|=m$.

\section{B. Dynamic calculation of the charge}

A dynamical alternative to computing the induced fermionic charge through the integrated local density of states is 

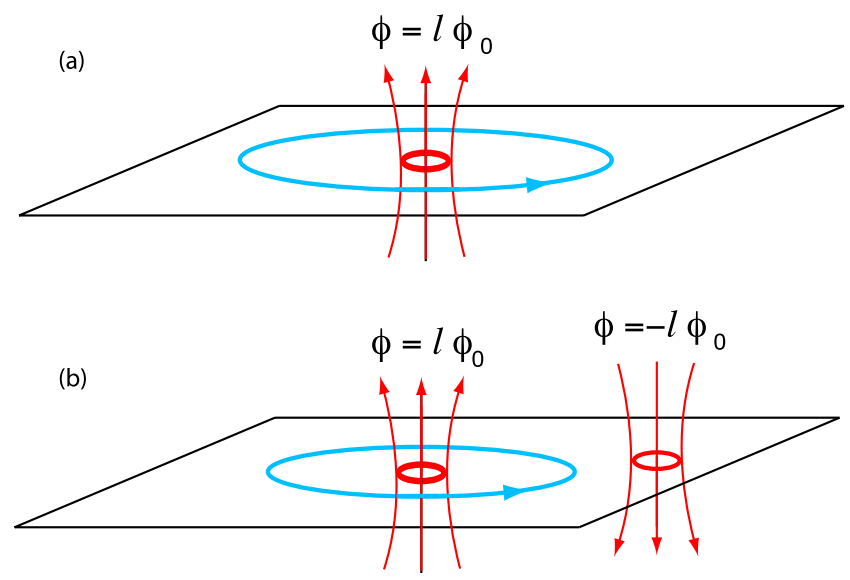

FIG. 3. (Color online) Schematics of the static magnetic flux tubes inserted to probe the induced fermionic charge of a quasiparticle, a mass vortex with or without the attachment of an axial gauge half flux, using the Aharonov-Bohm effect in the second setup described in the text. (a) We insert one static magnetic flux tube (colored in red) with the flux $\phi=l \phi_{0}$ (the flux quantum is $\phi_{0}=h c / e$ ) while a quasiparticle encircles dynamically this magnetic flux with the trajectory indicated by the directed loop (colored in blue). (b) We insert two static magnetic flux tubes (colored in red) with the fluxes $\phi= \pm l \phi_{0}$ while a quasiparticle encircles dynamically one and only one magnetic flux tube with the trajectory indicated by the directed loop (colored in blue).

the following. If we take a unit vortex in $\Delta$ (with or without an accompanying axial gauge half flux) around a circle of radius $r$ that encircles a magnetic flux, then an AharonovBohm phase accumulates. The value of the charge induced near the vortex follows after matching the Berry phase computed numerically to the analytical value of the Aharonov phase.

We carry out this approach in two different setups. In the first, we apply a uniform magnetic field to the system, i.e., we fix a given electromagnetic flux

$$
l \phi_{0}, \quad l \in \mathbb{R}, \quad \phi_{0} \text { the quantum of flux, }
$$

per elementary unit cell on the lattice. The Aharonov-Bohm phase $\gamma_{\mathrm{AB}}$ that is picked up depends on the radius of the path since the encircled magnetic flux scales with the area. The Aharonov-Bohm phase in this case is thus given by

$$
\gamma_{\mathrm{AB}}=2 \pi \times Q \times\left(\pi r^{2}\right) \times l .
$$

Here, $Q$ is the charge bound to the unit vortex in $\Delta$.

A second setup is shown in Fig. 3(a). We insert an electromagnetic flux tube with flux

$$
l \phi_{0}, \quad l \in \mathbb{R}, \quad \phi_{0} \text { the quantum of flux, }
$$

through the elementary unit cell on the lattice at the center of the system. All other elementary unit cells are free of any magnetic flux. We then move the unit vortex in the bonddensity-wave (Kekulé for graphene) order parameter around a path enclosing this flux. Notice that the Aharonov-Bohm phase $\gamma_{\mathrm{AB}}$ is independent of the path as long as it strictly contains the magnetic flux tube, i.e., the elementary unit cell at the center of the lattice. It is expected to have the value

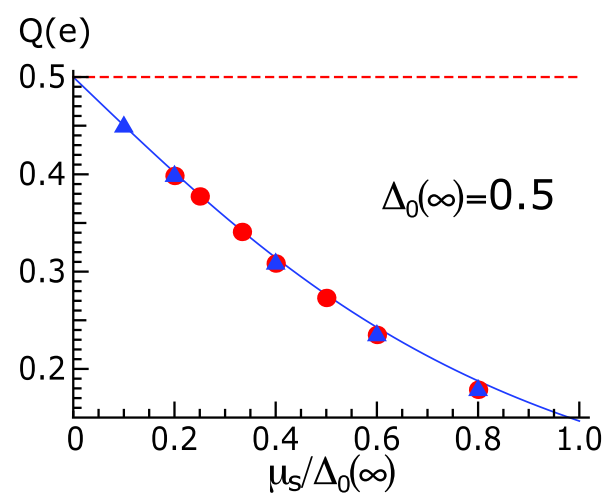

FIG. 4. (Color online) The induced fermionic charge of a quasiparticle, a unit vortex in $\Delta(\boldsymbol{r})$ with or without attachment of an axial gauge half flux in $\boldsymbol{a}_{5}(\boldsymbol{r})$ as a function of the ratio $\mu_{\mathrm{s}} / \Delta_{0}(\infty)$. This charge is obtained by matching the numerical Berry phase picked up when a quasiparticle hops along the closed boundary of an area that encloses a magnetic flux in the uniform background of $\mu_{\mathrm{s}}$ to the corresponding Aharonov-Bohm phase along the lines outlined in Sec. VII B and Appendix C. Hopping takes place on the square lattice with the lattice spacing $\mathfrak{a}$ and a magnetic flux of $\pi$ in units of the flux quantum $\phi_{0}=h c / e$ per elementary plaquette, there being $56 \times 56$ elementary plaquettes. The closed path used to compute the Berry phase is approximately circular with the radius $r$ $=14.5$ in units of the lattice spacing. The following parameters were chosen: the hopping $t=1$, the magnitude of $\Delta(\boldsymbol{r}, t)$ on the boundary is $\Delta_{0}(\infty)=0.5$, while the flux is $\phi=0.001 \phi_{0}$ through each elementary plaquette. The (red) filled circles are the induced charges of a dynamical unit vortex in $\Delta(\boldsymbol{r}, t)$ to which is also attached a dynamical axial gauge half flux in $\boldsymbol{a}_{5}(\boldsymbol{r}, t)$ as a function of $\mu_{\mathrm{s}} / \Delta_{0}(\infty)$. The (red) dashed line is the analytical charge $Q=1 / 2$. The (blue) filled triangles are the induced charges of a dynamical unit vortex in $\Delta(\boldsymbol{r}, t)$ without the axial gauge half flux in $\boldsymbol{a}_{5}(\boldsymbol{r})$ as a function of the ratio $\mu_{\mathrm{s}} / \Delta_{0}(\infty)$. The (blue) solid line is the induced charge computed from Eq. (5.7) as a function of the ratio $\mu_{\mathrm{s}} / \Delta_{0}(\infty)$.

$$
\gamma_{\mathrm{AB}}=2 \pi \times Q \times l .
$$

We also study the case displayed in Fig. 3(b). The reason for it is that we want to ensure that compensating fermionic charges on the edges of the sample do not contribute a phase as well. In the set up of Fig. 3(b), whatever happens with the fermionic edge charges does not lead to an Aharonov phase because their path would encircle (even if they move) the vanishing total flux,

$$
\phi=l \phi_{0}-l \phi_{0}=0 \text {. }
$$

The results we obtain for the Berry phase when we wind the unscreened vortices around a closed path are shown in Fig. 4 for the case of the first setup (uniform applied magnetic field). We fix the parameters $\Delta=0.5, r=14.5$ (in a $56 \times 56$ lattice), and $\phi=0.001 \phi_{0}$ per plaquette and plot the charge $Q$ versus the parameter $\mu_{\mathrm{s}} / \Delta$. The blue dots and red dots are the numerical results for a vortex without the axial gauge half flux and with the axial gauge half flux, respectively, while the corresponding theoretical predictions from Ref. 15 and 16 are plotted in blue and red solid line. Notice that the analytical and numerical results agree quite 
well for the case of vortices unscreened by axial gauge half fluxes.

As anticipated, the analytical and numerical results are not consistent for the case of screened vortices. The reason is precisely what we highlighted in the beginning of this section, i.e., that the lattice model studied numerically in this section does not contain the $\mathrm{U}(1) \times \mathrm{U}(1)$ symmetry, i.e., the axial gauge field dynamics present in Dirac Hamiltonian (2.1a), (2.1b), (2.1c), (2.1d), and (2.1e). The same issue applies to the problem of computing the exchange statistics of pairs of screened vortices. We cannot study the statistical angle of screened vortices within the approach of this section. In Sec. VIII, we will present a microscopic model that does have the $\mathrm{U}(1) \times \mathrm{U}(1)$ gauge symmetry. However, this model cannot be studied by simply computing Slater determinants (see Appendix C) as has been done so far in this section.

Before closing Sec. VII B, let us mention that we have checked the results summarized by Fig. 4 that we obtained by applying a uniform magnetic field against those obtained with a single flux tube as in Fig. 3(a) or with two flux tubes as in Fig. 3(b).

\section{Fractional statistics for unscreened vortices}

We now present the numerical value of the statistical angle $\Theta$ in units of $\pi$ acquired under the exchange of two unit unscreened vortices in the bond-density-wave (Kekulé for graphene) order parameter $\Delta$, which we shall call quasiparticles from now on. We have computed numerically the Berry phase $\gamma$ in units of $\pi$ accumulated when a first dynamical quasiparticle moves along a trajectory that winds once around a second static quasiparticle as outlined in Appendix C. The statistical angle $\Theta$ acquired under the exchange between these two quasiparticles is then

$$
\Theta=\frac{\gamma}{2}
$$

Here, as we do not impose dynamically the axial gauge symmetry at the microscopic level as presented in Appendix C and unlike in Sec. VIII, we only treat unscreened vortices. We have verified that $\gamma$, when computed along the lines of Appendix $\mathrm{C}$, does not change when axial gauge half flux tubes are attached to the vortices.

To compare the microscopic exchange statistics with the one computed within field theory in Sec VI B, we restrict the numerical computation to the half-filled case. However, we will also test the prediction of Sec. VIC by working with one spinless fermion more than (or less than) at half-filling.

We will always take $m \approx 0.5$ in Eq. (2.2). Moreover, to limit finite size effects, we assume that $\eta \ll m$, i.e., we work well below the transition point $|\eta|=m$ when the breaking of TRS is weak.

The $\eta$ dependence of the Berry phase $\gamma$ with $m$ fixed is shown in Fig. 5 for different values of the uniform staggered chemical potentials $\mu_{\mathrm{s}}$. The magnitude of the Berry phase is seen to be independent of whether the pair of quasiparticles have the same (filled circles) or opposite (star symbols) vorticities, but it does depend on $\mu_{\mathrm{s}}$, i.e., on the induced frac-

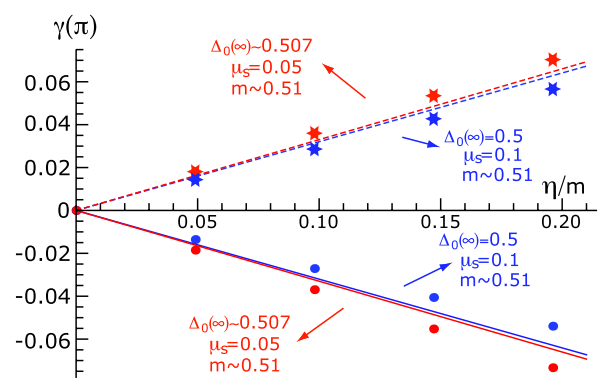

FIG. 5. (Color online) Berry phase in units of $\pi$ as a function of $\eta / m \ll 1$ for fixed $m$ acquired during the exchange of two unscreened quasiparticles, i.e., unit vortices in $\Delta(\boldsymbol{r}, t)$ without the attachment of axial gauge half fluxes in $\boldsymbol{a}_{5}(\boldsymbol{r}, t)$. Numerical computations along the lines outlined in Sec. VII B and Appendix C were performed for spinless fermions hopping on the square lattice with lattice spacing $\mathfrak{a}$ and with a magnetic flux of $\pi$ in units of the flux quantum $\phi_{0}=h c / e$ threading each elementary plaquette in the $d y$ namic background of a unit vortex in $\Delta(\boldsymbol{r}, t)$ without the attachment of an axial gauge half flux in $\boldsymbol{a}_{5}(\boldsymbol{r}, t)$ and for a uniform value of $\eta$. The square lattice is $72 \times 72$ and the exchange path is approximately circular with the radius $r=18.5$ in units of the lattice spacing. The following parameters were chosen: the hopping $t=1, m$ $=\sqrt{\Delta_{0}^{2}(\infty)+\mu_{\mathrm{s}}^{2}} \approx 0.51$ but with two different value of $\mu_{\mathrm{s}}=0.1$ and 0.025 . Filled circles and solid lines represent the case when the two quasiparticles carry the same unit vorticity. Stars and dashed lines represent the case when the two quasiparticles carry the opposite unit vorticity. Symbols are obtained numerically while the lines are the predictions from Sec. VI B.

tional charge $Q$ given in Eq. (5.7). The $\eta$ dependence of $\gamma$ is linear, as predicted in Sec. VI B, but with slopes deviating from the theoretical predictions, i.e., Eq. (6.16), shown as the solid or dashed lines. The agreement between the Berry phase of the microscopic model and Eq. (6.16) is thus qualitatively but not quantitatively good.

The microscopic Berry phase $\gamma$ as a function of the ratio $\Delta / m$, which also parametrizes $Q\left(\Delta, \mu_{\mathrm{s}}\right)$, when $\eta=0.025$ is held fixed is shown in Fig. 6 as filled circles when the quasiparticles carry the same vorticities or as stars when the quasiparticles carry the opposite vorticities. As expected, exchanging a pair of quasiparticles with equal unit vorticities differs solely by a sign relative to exchanging a pair of quasiparticles with opposite unit vorticities. The lines (solid when the quasiparticles have the same unit vorticity, dashed otherwise) are given by Eq. (6.16). Evidently, the dependence on $Q \ll 1 / 2$ of the microscopic exchange statistics is not captured by the field theory.

As discussed in Sec. VI C, when adding (removing) one fermion to (from) half-filling, the Berry phase accumulated by a complete winding of quasiparticles of opposite unit vorticities changes by $\pi$ for the case $\eta=0$. This extra phase is the response of the single-particle midgap states to varying the phase of $\Delta$ by $2 \pi$. Numerically, this assertion is confirmed directly by computing the accumulated Berry phase and obtaining $\gamma= \pm \pi$ when filling or emptying one midgap state.

In summary, comparison of the microscopic Berry phase accumulated by winding an unscreened quasiparticle around a static one with the field-theory computation of the ex- 


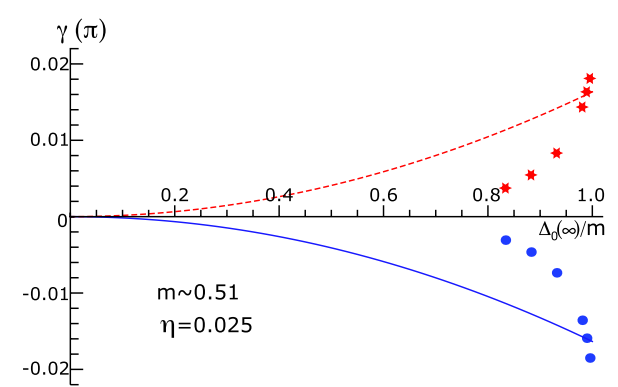

FIG. 6. (Color online) Berry phase in units of $\pi$ as a function of $\Delta_{0}(\infty) / m$ for fixed $m$ and $\eta \ll m$ acquired during the exchange of two unscreened quasiparticles, i.e., unit vortices in $\Delta(\boldsymbol{r}, t)$ without the attachment of axial gauge half fluxes in $\boldsymbol{a}_{5}(\boldsymbol{r}, t)$. Numerical computations along the lines outlined in Sec. VII B and Appendix C were performed for spinless fermions hopping on the square lattice with lattice spacing $\mathfrak{a}$ and with a magnetic flux of $\pi$ in units of the flux quantum $\phi_{0}=h c / e$ threading each elementary plaquette in the dynamic background of a unit vortex in $\Delta(\boldsymbol{r}, t)$ without the attachment of an axial gauge half flux in $\boldsymbol{a}_{5}(\boldsymbol{r}, t)$ and for a uniform value of $\eta$. The square lattice is $72 \times 72$ and the exchange path is approximately circular with the radius $r=18.5$ in units of the lattice spacing. The following parameters were chosen: the hopping $t=1$, $m=\sqrt{\Delta_{0}^{2}(\infty)+\mu_{\mathrm{s}}^{2}}=0.51$ and $\eta=0.025$. Filled circles and solid lines represent the case when the two quasiparticles carry the same unit vorticity. Stars and dashed lines represent the case when the two quasiparticles carry the opposite unit vorticity. Symbols are obtained numerically while the lines are the predictions from Sec. VI B.

change statistic in Sec. VI B shows the following. (1) The microscopic Berry phase $\gamma$ (and consequently the microscopic exchange statistical angle $\Theta=\gamma / 2$ ) varies continuously as a function of $\eta$ and in a linear fashion for small $\eta$, in good agreement with the field-theory results. (2) The slope $\gamma / \eta$ shows a monotonic dependence on the ratio $\Delta / m$, which is not in good quantitative agreement with the field-theory results. (3) The magnitude $|\gamma|$ is independent of the relative sign of the quasiparticles vorticities. This is expected for a vortex and its anti-vortex can annihilate. Consequently, winding a third vortex around a vortex antivortex pair must accumulate a vanishing Berry phase. (4) Microscopic semion statistics $\Theta= \pm \pi / 2$ is obtained when adding (removing) one fermion to (from) the half-filled system in agreement with the prediction from the continuum theory.

\section{MICROSCOPIC MODEL}

We have seen in Sec. VII that the fractional charge induced by an axial gauge half flux in $\boldsymbol{a}_{5}$ cannot be measured dynamically from the Aharonov-Bohm phase inferred from the numerical computation of a Berry phase. This is so because the local axial gauge symmetry in the continuum Hamiltonian (2.1a), (2.1b), (2.1c), (2.1d), and (2.1e) is not present in the lattice model used in Sec. VII. Thus, there is a dynamical contribution that is missing and that cannot be captured by the simple models of one species of fermions hopping either on the honeycomb or $\pi$-flux lattices used in Sec. VII. For the same reason, we could not obtain numeri- cally the exchange statistics in the case when the vortices are screened by the axial gauge potential since the exchange of the topological defects necessarily acquires a dynamical contribution from $\boldsymbol{a}_{5}$.

We now construct a lattice model sharing the same local $\mathrm{U}(1) \times \mathrm{U}(1)$ symmetry and the same particle content as dynamical theory (3.1a), (3.1b), (3.1c), and (3.1d). The predictions for the exchange statistics of screened vortices done in Sec. VI A should be captured by this lattice model. Unfortunately, we cannot verify this claim for the largest system sizes that we could treat numerically are of the order of the vortex core.

Consider a square lattice $\Lambda$ whose sites we denote with the Latin letters $i, j, k$, and $l$. We denote with $\hat{1} \equiv \hat{\mathbf{x}}$ and $\hat{2} \equiv \hat{\mathbf{y}}$ the two orthonormal vectors spanning the square lattice $\Lambda$ (and we will index these two vectors as $\hat{\mu}=\hat{1}, \hat{2}$ for $\mu=1,2$ ). Links (or bonds) on the square lattice between nearest-neighbor sites $i$ and $j$ are labeled by $\langle i j\rangle$ (or simply by $i j$ when used as an index to a field defined on the links). We denote by $\square_{i j k l}$ the square plaquette with the corners $i, j$, $k$, and $l$.

We define four sets of operators. There are the bosonic operators $\hat{A}_{i j}$ and $\hat{A}_{5 i j}$ living on the links of the square lattice $\Lambda$. There are the bosonic operators $\hat{\phi}_{i}$ and the fermionic operators $\hat{\psi}_{i}$ living on the sites. The spinor-valued operator $\hat{\psi}_{i}$ has here four components on which the $4 \times 4$ matrices defined in Eqs. (2.1d) and (2.1e) act.

These four sets of operators, together with their canonical conjugate operators, satisfy the following relations:

$$
\begin{gathered}
\hat{A}_{k l}^{\dagger}=\hat{A}_{k l}=-\hat{A}_{l k}, \quad \hat{L}_{i j}^{\dagger}=\hat{L}_{i j}=-\hat{L}_{j i}, \\
{\left[\hat{L}_{i j}, \hat{A}_{k l}\right]=-i\left(\delta_{i k} \delta_{j l}-\delta_{i l} \delta_{j k}\right),} \\
\hat{A}_{5 k l}^{\dagger}=\hat{A}_{5 k l}=-\hat{A}_{5 l k}, \quad \hat{L}_{5 i j}^{\dagger}=\hat{L}_{5 i j}=-\hat{L}_{5 j i}, \\
{\left[\hat{L}_{5 i j}, \hat{A}_{5 k l}\right]=-i\left(\delta_{i k} \delta_{j l}-\delta_{i l} \delta_{j k}\right),} \\
\hat{\phi}_{j}^{\dagger}=\hat{\phi}_{j}, \quad \hat{\Pi}_{i}^{\dagger}=\hat{\Pi}_{i}, \quad\left[\hat{\Pi}_{i}, \hat{\phi}_{j}\right]=-i \delta_{i j},
\end{gathered}
$$

and, finally,

$$
\left\{\hat{\psi}_{i}, \hat{\psi}_{j}^{\dagger}\right\}=\mathbb{1}_{4} \delta_{i j}, \quad\left\{\hat{\psi}_{i}^{\dagger}, \hat{\psi}_{j}^{\dagger}\right\}=\left\{\hat{\psi}_{j}, \hat{\psi}_{i}\right\}=0,
$$

with the equal-time global constraint (half-filling constraint),

$$
|\Lambda|^{-1} \sum_{i \in \Lambda} \hat{\psi}_{i}^{\dagger} \hat{\psi}_{i}=2
$$

(Since we are working with four flavors of fermions, halffilling means average two particles per each site.)

We define the lattice model by the quantum Hamiltonian,

$$
\hat{H}:=\hat{H}_{g}+\hat{H}_{g_{5}}+\hat{H}_{J}+\hat{H}_{t}+\hat{H}_{t^{\prime}}+\hat{H}_{m} .
$$

Here, 


$$
\hat{H}_{g}:=\frac{g^{2}}{2} \sum_{\langle i j\rangle} \hat{L}_{i j}^{2}-\frac{1}{g^{2}} \sum_{\square_{i j k l}} \operatorname{Re} e^{i\left(\hat{A}_{i j}+\hat{A}_{j k}+\hat{A}_{k l}+\hat{A}_{l i}\right)}
$$

describes a U(1) lattice gauge theory with gauge coupling $g^{2}$,

$$
\hat{H}_{g_{5}}:=\frac{g_{5}^{2}}{2} \sum_{\langle i j\rangle} \hat{L}_{5 i j}^{2}-\frac{1}{g_{5}^{2}} \sum_{\square_{i j k l}} \operatorname{Re} e^{i\left(\hat{A}_{5 i j}+\hat{A}_{5 j k}+\hat{A}_{5 k l}+\hat{A}_{5 l i}\right)}
$$

describes another $\mathrm{U}(1)$ lattice gauge theory with gauge coupling $g_{5}^{2}$,

$$
\hat{H}_{J}:=\frac{J^{2}}{2} \sum_{i \in \Lambda} \hat{\Pi}_{i}^{2}-\frac{1}{J^{2}} \sum_{\langle i j\rangle}\left(e^{+i\left(\hat{\phi}_{i}-\hat{\phi}_{j}\right)+2 i \hat{A}_{5 i j}}+\text { H.c. }\right)
$$

describes a quantum rotor $(X Y)$ model with coupling $J^{2}$, and

$$
\hat{H}_{t}:=i t \sum_{i \in \Lambda} \sum_{\mu=1,2} \hat{\psi}_{i}^{\dagger} \alpha_{\mu} e^{i \hat{A}_{i(i+\hat{\mu})}+i \gamma_{5} \hat{A}_{5 i(i+\hat{\mu})}} \hat{\psi}_{(i+\hat{\mu})}+\text { H.c. }
$$

describes the nearest-neighbor hopping with the real-valued amplitude $t$ of four independent fermions per site. So far, there are four nonequivalent Dirac points at half-filling which are located at $k=(0,0),(0, \pi),(\pi, 0)$, and $(\pi, \pi)$. This is why we have added the term

$$
\begin{aligned}
\hat{H}_{t^{\prime}}:= & t^{\prime} \sum_{i \in \Lambda} \\
& \times\left[\hat{\psi}_{i}^{\dagger} 4 R \hat{\psi}_{i}-\sum_{\mu=1,2}\left(\hat{\psi}_{i}^{\dagger} R e^{i \hat{A}_{i(i+\hat{\mu})}+i \gamma_{5} \hat{A}_{5 i}(i+\hat{\mu})} \hat{\psi}_{(i+\hat{\mu})}+\text { H.c. }\right)\right]
\end{aligned}
$$

that opens a gap of order $t^{\prime}$ at the points $k=(0, \pi),(\pi, 0)$, $(\pi, \pi)$, thus leaving $k=(0,0)$ as the sole Dirac point. This scheme is precisely Wilson's procedure used to overcome the doubling problem in lattice gauge theories ${ }^{47}$ An important comment is in order, however. One reason why this prescription is not fully satisfying in lattice gauge theories is that any mismatch between the first and second terms of Eq. (8.2f) leads to a gap at $\boldsymbol{k}=(0,0)$ as well; i.e., fine tuning is needed to achieve the correct particle content. Here, this is fine because we are interested in systems where there is such a gap. Notice in that regard that the gap at $\boldsymbol{k}=(0,0)$ that arises from a small mismatch between these two terms (a small fraction of $t^{\prime}$ ) is much smaller than the one at the edges of the Brillouin zone (order $t^{\prime}$ ). Indeed, such a term due to a mismatch is actually part of the final term that we consider in the Hamiltonian, namely,

$$
\hat{H}_{m}:=\sum_{i \in \Lambda} \hat{\psi}_{i}^{\dagger}\left(\mu_{\mathrm{s}} R+\Delta_{\mathrm{k}} \beta e^{i \gamma_{5} \hat{\phi}_{i}}+i \eta \alpha_{1} \alpha_{2}\right) \hat{\psi}_{i} .
$$

This contribution does indeed open a gap at the remaining Dirac point at $\boldsymbol{k}=(0,0)$.

For any smooth and static boson background, the continuum limit of Hamiltonian (8.2a), (8.2b), (8.2c), (8.2d), $(8.2 \mathrm{e}),(8.2 \mathrm{f})$, and $(8.2 \mathrm{~g})$ upon linearization of the fermion spectrum at the two nonequivalent Dirac points at half-filling is given by Eq. (2.1a), (2.1b), (2.1c), (2.1d), and (2.1e), as is also the case with the fermion spectrum of graphene re- stricted to spinless fermions hopping with sufficiently smooth modulations of the hopping amplitudes.

Contrary to graphene for spinless fermions, Hamiltonian (8.2a), (8.2b), (8.2c), (8.2d), (8.2e), (8.2f), and (8.2g) is invariant under the local $\mathrm{U}(1) \times \mathrm{U}(1)$ gauge transformation,

$$
\begin{gathered}
\hat{L}_{i j} \rightarrow \hat{L}_{i j}, \quad \hat{A}_{i j} \rightarrow \hat{A}_{i j}-\left(\chi_{i}-\chi_{j}\right), \\
\hat{L}_{5 i j} \rightarrow \hat{L}_{5 i j}, \quad \hat{A}_{5 i j} \rightarrow \hat{A}_{5 i j}-\left(\xi_{i}-\xi_{j}\right), \\
\hat{\Pi}_{i} \rightarrow \hat{\Pi}_{i}, \quad \hat{\phi}_{i} \rightarrow \hat{\phi}_{i}+2 \xi_{i}, \\
\hat{\psi}_{i}^{\dagger} \rightarrow \hat{\psi}_{i}^{\dagger} e^{+i \chi_{i}+i \gamma_{5} \xi_{i}}, \quad \hat{\psi}_{j} \rightarrow \psi_{j} e^{-i \chi_{j}-i \gamma_{5} \xi_{j},}
\end{gathered}
$$

generated by

$$
\hat{H} \rightarrow \hat{G}(\chi, \xi) \hat{H} \hat{G}^{-1}(\chi, \xi)
$$

with

$$
\begin{aligned}
\hat{G}(\chi, \xi):= & \prod_{i \in \Lambda} \exp \left[i\left(\hat{\psi}_{i}^{\dagger} \hat{\psi}_{i}+\sum_{\mu=1,2} \hat{L}_{i(i+\hat{\mu})}\right) \chi_{i}\right. \\
& \left.+i\left(\hat{\psi}_{i}^{\dagger} \gamma_{5} \hat{\psi}_{i}+2 \hat{\Pi}_{i}+\sum_{\mu=1,2} \hat{L}_{5 i(i+\hat{\mu})}\right) \xi_{i}\right]
\end{aligned}
$$

where $\chi_{i}$ and $\xi_{i}$ are arbitrary real-valued numbers.

The physical subspace is the set of gauge invariant states, i.e., states that are tensor products of states in the Fock space generated by the algebra Eqs. (8.1a), (8.1b), (8.1c), (8.1d), and (8.1e),

$$
|\Psi\rangle \equiv\left|\Psi_{A}\right\rangle \otimes\left|\Psi_{A_{5}}\right\rangle \otimes\left|\Psi_{\phi}\right\rangle \otimes\left|\Psi_{\psi}\right\rangle,
$$

such that Gauss law holds globally,

$$
\hat{G}^{-1}(\chi, \xi)|\Psi\rangle=|\Psi\rangle
$$

for all real-valued function $\chi$ and $\xi$, or, equivalently, locally

$$
\begin{gathered}
0=\left(\hat{L}_{i(i+1)}-\hat{L}_{i(i-\hat{1})}+\hat{L}_{i(i+\hat{2})}-\hat{L}_{i(i-\hat{2})}+\hat{\psi}_{i}^{\dagger} \hat{\psi}_{i}\right)|\Psi\rangle, \\
0=\left(\hat{L}_{5 i(i+\hat{1})}-\hat{L}_{5 i(i-1)}+\hat{L}_{5 i(i+2)}-\hat{L}_{5 i(i-2)}+\hat{\psi}_{i}^{\dagger} \gamma_{5} \hat{\psi}_{i}+2 \hat{\Pi}_{i}\right)|\Psi\rangle
\end{gathered}
$$

for any $i \in \Lambda$.

We denote by $\left|\Psi_{i, j}\right\rangle$ a gauge invariant state (8.4a), (8.4b), and $(8.4 \mathrm{c})$ with two fractional charges localized around sites $i$ and $j$, respectively. The statistical phase $\Theta$ induced by the physical process by which two fractional charges are exchanged is given by the difference between two Berry phases, ${ }^{48}$

$$
\Theta:=\frac{1}{2} \arg \prod_{i_{\iota} \in \mathcal{P}}^{j \subset \mathcal{P}}\left\langle\Psi_{i_{\iota+1}, j}|\hat{H}| \Psi_{i_{\iota}, j}\right\rangle-\frac{1}{2} \arg \prod_{i_{\iota} \in \mathcal{P}}^{j \subset \overline{\mathcal{P}}}\left\langle\Psi_{i_{\iota+1}, j}|\hat{H}| \Psi_{i_{\iota}, j}\right\rangle .
$$

For both Berry phases, one fractional charge hops along the closed path $\mathcal{P}=\left\{i_{\iota}\right\}$, while the other fractional charge is static. For the former Berry phase, $j$ is located inside the area bounded by $\mathcal{P}$, a choice that we denote by $j \subset \mathcal{P}$. For the 
latter Berry phase, $j$ is located outside the area bounded by $\mathcal{P}$, a choice that we denote by $j \subset \overline{\mathcal{P}}$.

The dimensionality of the gauge-invariant Hilbert space scales with the dimensionality of the fermionic Hilbert space (8.1d), which itself scales exponentially fast with the number of sites. Given the half-filling constraint (8.1e), this limits the numerical evaluation of the right-hand side of Eq. (8.5) to lattices with linear dimensions of the order of the core size $1 / m$ of the defects, i.e., on distances much too short for the right-hand side of Eq. (8.5) to be interpreted as the statistical angle of pointlike quasiparticles.

If we are willing to give up the local $\mathrm{U}(1) \times \mathrm{U}(1)$ gauge invariance $(8.3 \mathrm{a}),(8.3 \mathrm{~b})$, and $(8.3 \mathrm{c})$, i.e., the strongly correlated nature of the problem, we can compute the contribution to the statistical phase arising from the fermion hopping. Indeed, the problem then reduces to a single-particle one for which the dimensionality of the relevant Hilbert spaces only scales linearly with the number of sites. We stress that this contribution alone violates the local $\mathrm{U}(1) \times \mathrm{U}(1)$ gauge invariance.

\section{MORE SPECIES OF FERMIONS-CLASSIFICATION OF ALL MASSES IN GRAPHENE AND $\pi$-FLUX PHASE}

So far we have ignored the spin-1/2 quantum number of electrons. If so, in the linear approximation (2.1a), (2.1b), (2.1c), (2.1d), and (2.1e) of graphene restricted to spinless fermions, say, $\hat{H}_{\text {scalar }}$ exhausts all possible symmetrybreaking instabilities with a local order parameters compatible with charge conservation. The local order parameter for a charge-density wave that breaks the sublattice symmetry but preserves the time-reversal symmetry is the real-valued order parameter $\mu_{\mathrm{s}}(\boldsymbol{r})$ (introduced by Semenoff for graphene in Ref. 8). The local order parameter for a bond-density wave instability that preserves the sublattice and time-reversal symmetries is the complex-valued order parameter $\Delta(\boldsymbol{r})$ [the U(1) Kekulé order parameter introduced by Hou et al. for graphene in Ref. 10]. The local order parameter for a bonddensity wave instability that breaks the sublattice and timereversal symmetries is the real-valued order parameter $\eta(\boldsymbol{r})$ (introduced by Haldane for graphene in Ref. 9).

If we reinstate spin-1/2 in the most naive way and consider two independent copies of the model in Eq. (2.1a), (2.1b), (2.1c), (2.1d), and (2.1e), then the results we found for spinless electrons are modified in a trivial way. Defects bind equal values for the fractional charge for both species, up and down spin, thereby doubling the total induced fermionic charge (which is to be associated with a spin-singlet state). The same happens to the exchange statistical angle. It is simply doubled with respect to the results in Sec. VI.

However, if spin is not a good quantum number, a larger number of instabilities can occur and more masses or order parameters (other than $\operatorname{Re} \Delta, \operatorname{Im} \Delta, \mu_{\mathrm{s}}$, and $\eta$ ) need to be taken into account. Thus, one must consider more generic Dirac Hamiltonians and study all their allowed masses. Topological defects in these order parameters could bind states, whose (fractional) charge and statistics would depend on the effective action (as a function of all the mass order param- eters and the $a_{\mu}$ and $a_{5 \mu}$ fields) that is obtained upon integrating all the species of fermions. This effective action would be the extension of the one derived in Sec. IV for the case of the four order parameters $\left(\operatorname{Re} \Delta, \operatorname{Im} \Delta, \mu_{\mathrm{s}}\right.$, and $\left.\eta\right)$.

We do not fully carry this program in this paper. Nonetheless, we classify all these masses according to the microscopic symmetries.

This classification applies as well to the microscopic model of Sec. VIII. There, we chose a specific way to add Wilson masses [see Eq. (8.2f)] to selectively get rid of all but two Dirac points in order to recover in the long-wavelength limit Hamiltonian (2.1a), (2.1b), (2.1c), (2.1d), and (2.1e). The set of all (64) Wilson masses can also be classified as we do below.

\section{A. Classification of masses in graphene and $\pi$-flux phases}

To describe all symmetry-breaking instabilities with a local order parameter in graphene or the square lattice with $\pi$-flux phase, we consider the Bogoliubov-de Gennes (BdG) Hamiltonian,

$$
\hat{H}_{\mathrm{BdG}}=\frac{1}{2} \int d^{2} \boldsymbol{r} \hat{\Psi}^{\dagger} \mathcal{K} \hat{\Psi},
$$

where $\hat{\Psi}$ is the 16-component Nambu spinor,

$$
\hat{\Psi}:=\left(\begin{array}{lll}
\hat{\psi}_{\uparrow}, & \hat{\psi}_{\downarrow}, \quad \hat{\psi}_{\uparrow}^{\dagger}, \quad \hat{\psi}_{\downarrow}^{\dagger}
\end{array}\right)^{\mathrm{t}},
$$

and $\hat{\psi}_{s=\uparrow, \downarrow}$ is a four-component fermion annihilation operator that accounts for the two valley and the two sublattice degrees of freedom. The kernel of the BdG Hamiltonian has the block structure,

$$
\mathcal{K}=\left(\begin{array}{cc}
\mathcal{H}_{\mathrm{pp}} & \mathcal{H}_{\mathrm{ph}} \\
\mathcal{H}_{\mathrm{ph}}^{\dagger} & -\mathcal{H}_{\mathrm{pp}}^{\mathrm{t}}
\end{array}\right),
$$

where the $8 \times 8$ blocks $\mathcal{H}_{\mathrm{pp}}$ and $\mathcal{H}_{\mathrm{ph}}$ act on the combined space of valley, sublattice, and spin degrees of freedom and represent the normal and anomalous part of the BdG Hamiltonian, respectively. These blocks satisfy

$$
\begin{gathered}
\mathcal{H}_{\mathrm{pp}}^{\dagger}=\mathcal{H}_{\mathrm{pp}} \quad \text { (Hermiticity), } \\
\mathcal{H}_{\mathrm{ph}}^{\mathrm{t}}=-\mathcal{H}_{\mathrm{ph}} \quad \text { (Fermi statistics). }
\end{gathered}
$$

To represent the single particle Hamiltonian $\mathcal{K}$, define the 256 16-dimensional Hermitian matrices,

$$
X_{\mu_{1} \mu_{2} \mu_{3} \mu_{4}}:=\rho_{\mu_{1}} \otimes s_{\mu_{2}} \otimes \sigma_{\mu_{3}} \otimes \tau_{\mu_{4}},
$$

where $\mu_{1,2,3,4}=0,1,2,3$. Here, we have introduced the four families $\rho_{\mu_{1}}, s_{\mu_{2}}, \sigma_{\mu_{3}}$, and $\tau_{\mu_{4}}$ of unit $2 \times 2$ and Pauli matrices that encode the particle-hole (Nambu), spin-1/2, valley, and sublattice degrees of freedom of graphene or the square lattice with $\pi$-flux phase, respectively.

The Dirac kinetic energy $\mathcal{K}_{0}$ of graphene or the square lattice with $\pi$-flux phase that accounts for the BdG block structure (9.1c) is assigned the two $16 \times 16$ Dirac matrices,

$$
\alpha_{1} \equiv X_{0031}, \quad \alpha_{2} \equiv X_{3032},
$$

and is given by 


$$
\mathcal{K}_{0}:=\boldsymbol{\alpha} \cdot(-i \boldsymbol{\partial}) .
$$

Similarly, by introducing the $16 \times 16$ Hermitian matrices,

$$
\beta \equiv X_{3010}, \quad R \equiv X_{3033}, \quad \gamma_{5} \equiv X_{3030},
$$

the counterpart to $\hat{H}$ in Eq. (2.1a), (2.1b), (2.1c), (2.1d), and (2.1e) is given by

$$
\mathcal{K}:=\mathcal{K}_{0}+\mathcal{K}_{\text {gauge }}+\mathcal{K}_{\text {scalar }},
$$

where

$$
\begin{gathered}
\mathcal{K}_{\text {gauge }}:=\boldsymbol{\alpha} \cdot\left(-\boldsymbol{a}-\boldsymbol{a}_{5} \gamma_{5}\right), \\
\mathcal{K}_{\text {scalar }}:=|\Delta| \beta e^{i \theta \gamma_{5}}+\mu_{\mathrm{s}} R+i \eta \alpha_{1} \alpha_{2} .
\end{gathered}
$$

Given the Dirac kinetic term $\mathcal{K}_{0}$, we treat $X_{\mu_{1} \mu_{2} \mu_{3} \mu_{4}}$ as a perturbation,

$$
\mathcal{K}_{m}:=\mathcal{K}_{0}+m X_{\mu_{1} \mu_{2} \mu_{3} \mu_{4}},
$$

where $m \in \mathbb{R}$ is constant in space and time. If $X_{\mu_{1} \mu_{2} \mu_{3} \mu_{4}}$ anticommutes with the Dirac kinetic energy $\mathcal{K}_{0}$, then it opens a gap in the massless Dirac spectrum of $\mathcal{K}_{0}$. We shall call such a perturbation a mass in short. Each mass can be thought of as being induced by a breaking of a microscopic symmetry (see below).

There are $64=4 \times 16$ mass matrices (i.e., $X_{\mu_{1} \mu_{2} \mu_{3} \mu_{4}}$ that anticommutes with $\mathcal{K}_{0}$ ). Of these 64 mass matrices, only 36 satisfy the condition

$$
X_{1000} X_{\mu_{1} \mu_{2} \mu_{3} \mu_{4}}^{\mathrm{t}} X_{1000}=-X_{\mu_{1} \mu_{2} \mu_{3} \mu_{4}}
$$

for particle-hole symmetry (PHS) and are thus compatible with the symmetry condition $\left(\rho_{1} \otimes s_{0} \otimes \sigma_{0} \otimes \tau_{0} \hat{\Psi}\right)^{\mathrm{t}}=\hat{\Psi}^{\dagger}$ on the Nambu spinors [i.e., compatible with Eq. (9.2)]. All mass matrices with PHS are enumerated in Table II.

All 36 mass matrices from Table II can be classified in terms of the following (microscopic) three symmetry properties. (i) A BdG Hamiltonian is time-reversal symmetry (TRS) when

$$
X_{0211} \mathcal{K}^{*} X_{0211}=\mathcal{K} .
$$

(ii) A BdG Hamiltonian has $\mathrm{SU}(2)$ spin rotation symmetry (SRS) when

$$
\left[X_{3100}, \mathcal{K}\right]=\left[X_{0200}, \mathcal{K}\right]=\left[X_{3300}, \mathcal{K}\right]=0 .
$$

(iii) A BdG Hamiltonian has sublattice symmetry (SLS) when

$$
X_{0033} \mathcal{K} X_{0033}=-\mathcal{K} .
$$

For any lattice regularization of the BdG Hamiltonian (9.5) supporting two sublattices $\Lambda_{\mathrm{A}}$ and $\Lambda_{\mathrm{B}}$, as is the case for graphene or the square lattice with $\pi$-flux phase, the microscopic order parameter corresponding to a mass matrix satisfying the SLS (9.9) is a nonvanishing expectation value for a fermion bilinear with the two lattice fermions residing on the opposite ends of a bond connecting a site belonging to sublattice $\Lambda_{\mathrm{A}}$ and another site belonging to sublattice $\Lambda_{\mathrm{B}}$. We shall say that such a mass matrix is associated to a valence- bond solid (VBS) order parameter in analogy to the terminology used for quantum dimer models. A VBS order picks up a microscopic orientation that translates into a complexvalued order parameter in the continuum limit. Hence, we shall distinguish between the real (Re VBS) and imaginary (Im VBS) parts of the VBS. Triplet superconductivity is also possible on bonds connecting the two sublattices. The terminology TSC will then also be used. To distinguish TSC with or without TRS we shall reserve the prefixes Re and Im for real and imaginary parts. This is a different convention for the use of the prefixes Re and Im than for a VBS.

Any mass matrix that does not satisfy the SLS (9.9) corresponds to a microscopic order parameter for which the fermion bilinear has the two lattice fermions sitting on the same sublattice. Microscopic examples are charge-density waves (CDWs), spin-density waves such as Néel ordering, orbital currents leading to the quantum Hall effect (QHE), spin-orbit couplings leading to the quantum spin Hall effect (QSHE), singlet superconductivity (SSC), or triplet superconductivity (TSC).

When $\mathrm{SU}(2)$ spin symmetry is broken by the order parameter, we add a subindex $x, y$, or $z$ that specifies the relevant quantization axis to the name of the mass matrix. Moreover, TSC with SLS must be distinguished by the two possible bond orientations (the underlying two-dimensional lattice has two independent vectors connecting nearest-neighbor sites). These two orientations are specified by the Pauli matrices used in the valley and sublattice subspaces, i.e., by the two pairs of numbers 02 and 32. Symmetry properties of all 36 PHS masses are summarized in Table II.

The set of all 36 PHS masses in Table II is invariant under an involutive transformation defined by

$$
\begin{gathered}
\hat{\Psi} \rightarrow C \hat{\Psi}, \\
C=\rho_{0} \otimes s_{+} \otimes \sigma_{0} \otimes \tau_{0}+\rho_{1} \otimes s_{-} \otimes \sigma_{2} \otimes \tau_{2},
\end{gathered}
$$

and which we shall call $C$ conjugation to distinguish it from the particle-hole transformation (9.6). Here, $s_{ \pm}=\left(s_{3} \pm s_{0}\right) / 2$. For graphene or the square lattice with $\pi$-flux phase, this transformation corresponds to

$$
\begin{gathered}
\hat{a}_{\boldsymbol{r}_{A} \uparrow} \rightarrow \hat{a}_{\boldsymbol{r}_{A} \uparrow}, \quad \hat{b}_{\boldsymbol{r}_{B} \uparrow} \rightarrow \hat{b}_{\boldsymbol{r}_{B} \uparrow}, \\
\hat{a}_{\boldsymbol{r}_{A} \downarrow} \rightarrow \hat{a}_{\boldsymbol{r}_{A} \downarrow}^{\dagger}, \quad \hat{b}_{\boldsymbol{r}_{B} \downarrow} \rightarrow-\hat{b}_{\boldsymbol{r}_{B} \downarrow}^{\dagger},
\end{gathered}
$$

where $\hat{a}_{r_{A} s}^{\dagger}$ and $\hat{b}_{r_{B} s}^{\dagger}$ creates an electron with spin $s=\uparrow, \downarrow$ on sublattice $\Lambda_{\mathrm{A}}$ and sublattice $\Lambda_{\mathrm{B}}$, respectively (see Appendix C). Under this transformation

$$
X_{\mu_{1} \mu_{2} \mu_{3} \mu_{4}} \rightarrow C^{\dagger} X_{\mu_{1} \mu_{2} \mu_{3} \mu_{4}} C .
$$

Hence, it leaves the massless Dirac kernel $\mathcal{K}_{0}$ invariant.

The organization of the mass matrices in Table II can be understood as follows. First, we preserve both SRS and charge conservation, i.e., we start with the four order 
TABLE II. The 36 mass matrices with particle-hole symmetry (PHS), see Eq. (9.6), for the massless Dirac Hamiltonian $\mathcal{K}_{0}$ from Eq. (9.4b) are of form (9.3) and anticommute with $\mathcal{K}_{0}$. Each mass matrix can be assigned an order parameter for the underlying microscopic model, here graphene or the square lattice with $\pi$-flux phase. The latin subindex of the order parameter's name corresponds to the preferred quantization axis in $\mathrm{SU}(2)$ spin space. The pair of numeral subindices 02 and 32 are used to distinguish the two unit vectors spanning two-dimensional space. Each mass matrix preserves or breaks time-reversal symmetry (TRS), see Eq. (9.7), spin-rotation symmetry (SRS), see Eq. (9.8), and sublattice symmetry (SLS), see Eq. (9.9). To any of the 36 mass matrices corresponds a "partner" mass matrix obtained through the involutive transformation (9.12) denoted $C$

\begin{tabular}{|c|c|c|c|c|c|c|c|}
\hline Mass matrix & Order parameter & TRS & SRS & SLS & Partner by $C$ & Order parameter by $C$ & $C$ invariant \\
\hline$X_{3010}$ & Re VBS & True & True & True & $X_{3010}$ & Re VBS & True \\
\hline$X_{0020}$ & Im VBS & True & True & True & $X_{0020}$ & Im VBS & True \\
\hline$X_{3033}$ & CDW & True & True & False & $X_{3333}$ & $\mathrm{Néel}_{z}$ & False \\
\hline$X_{3003}$ & QHE & False & True & False & $X_{3003}$ & QHE & True \\
\hline$X_{3110}$ & $\operatorname{Re} \operatorname{VBS}_{x}$ & False & False & True & $X_{2132}$ & $\operatorname{Im} \mathrm{TSC}_{32 z}$ & False \\
\hline$X_{0210}$ & $\operatorname{ReVBS}_{y}$ & False & False & True & $X_{1132}$ & $\operatorname{Re} \operatorname{TSC}_{32 z}$ & False \\
\hline$X_{3310}$ & $\operatorname{Re} V_{B S}$ & False & False & True & $X_{3310}$ & $\operatorname{Re} V_{B S}$ & True \\
\hline$X_{0120}$ & $\operatorname{Im} \operatorname{VBS}_{x}$ & False & False & True & $X_{1102}$ & $\operatorname{Re} \operatorname{TSC}_{02 z}$ & False \\
\hline$X_{3220}$ & $\operatorname{Im} \mathrm{VBS}_{y}$ & False & False & True & $X_{2102}$ & $\operatorname{Im~TSC}_{02 z}$ & False \\
\hline$X_{0320}$ & $\operatorname{Im} \mathrm{VBS}_{z}$ & False & False & True & $X_{0320}$ & $\operatorname{Im} \mathrm{VBS}_{z}$ & True \\
\hline$X_{3103}$ & $\mathrm{QSHE}_{x}$ & True & False & False & $X_{2121}$ & $\operatorname{Im} \mathrm{TSC}_{z}$ & False \\
\hline$X_{0203}$ & $\mathrm{QSHE}_{y}$ & True & False & False & $X_{1121}$ & $\operatorname{Re} \mathrm{TSC}_{z}$ & False \\
\hline$X_{3303}$ & $\mathrm{QSHE}_{z}$ & True & False & False & $X_{3303}$ & $\mathrm{QSHE}_{z}$ & True \\
\hline$X_{3133}$ & Néel $_{x}$ & False & False & False & $X_{2211}$ & Re SSC & False \\
\hline$X_{0233}$ & Néel $_{y}$ & False & False & False & $X_{1211}$ & Im SSC & False \\
\hline$X_{3333}$ & Néel $_{z}$ & False & False & False & $X_{3033}$ & CDW & False \\
\hline$X_{2211}$ & Re SSC & True & True & False & $X_{3133}$ & Néel $_{x}$ & False \\
\hline$X_{1211}$ & Im SSC & False & True & False & $X_{0233}$ & Néel $_{y}$ & False \\
\hline$X_{1002}$ & $\operatorname{Re} \mathrm{TSC}_{02 y}$ & True & False & True & $X_{1002}$ & $\operatorname{Re} \operatorname{TSC}_{02 y}$ & True \\
\hline$X_{2002}$ & $\operatorname{Im} \mathrm{TSC}_{02 y}$ & False & False & True & $X_{2302}$ & $\operatorname{Im} \operatorname{TSC}_{02 x}$ & False \\
\hline$X_{1102}$ & $\operatorname{Re} \mathrm{TSC}_{02 z}$ & False & False & True & $X_{0120}$ & $\operatorname{Im} \mathrm{VBS}_{x}$ & False \\
\hline$X_{2102}$ & $\operatorname{Im} \mathrm{TSC}_{02 z}$ & True & False & True & $X_{3220}$ & $\operatorname{Im} \mathrm{VBS}_{y}$ & False \\
\hline$X_{1302}$ & $\operatorname{Re} \operatorname{TSC}_{02 x}$ & False & False & True & $X_{1302}$ & $\operatorname{Re} \operatorname{TSC}_{02 x}$ & True \\
\hline$X_{2302}$ & $\operatorname{Im} \operatorname{TSC}_{02 x}$ & True & False & True & $X_{2002}$ & $\operatorname{Im} \mathrm{TSC}_{02 y}$ & False \\
\hline$X_{1032}$ & $\operatorname{Re} \operatorname{TSC}_{32 y}$ & False & False & True & $X_{1332}$ & $\operatorname{Re} \operatorname{TSC}_{32 x}$ & False \\
\hline$X_{2032}$ & $\operatorname{Im} \mathrm{TSC}_{32 y}$ & True & False & True & $X_{2032}$ & $\operatorname{Im} \mathrm{TSC}_{32 y}$ & True \\
\hline$X_{1132}$ & $\operatorname{Re} \mathrm{TSC}_{32 z}$ & True & False & True & $X_{0210}$ & $\operatorname{Re}_{V_{B S}}$ & False \\
\hline$X_{2132}$ & $\operatorname{Im~TSC}_{32 z}$ & False & False & True & $X_{3110}$ & $\operatorname{Re} \operatorname{VBS}_{x}$ & False \\
\hline$X_{1332}$ & $\operatorname{Re} \operatorname{TSC}_{32 x}$ & True & False & True & $X_{1032}$ & $\operatorname{Re} \operatorname{TSC}_{32 y}$ & False \\
\hline$X_{2332}$ & $\operatorname{Im} \operatorname{TSC}_{32 x}$ & False & False & True & $X_{2332}$ & $\operatorname{Im} \operatorname{TSC}_{32 x}$ & True \\
\hline$X_{1021}$ & $\operatorname{Re} \mathrm{TSC}_{y}$ & True & False & False & $X_{1321}$ & $\operatorname{Re} \operatorname{TSC}_{x}$ & False \\
\hline$X_{2021}$ & $\operatorname{Im~} \mathrm{TSC}_{y}$ & False & False & False & $X_{2021}$ & $\operatorname{Im} \operatorname{TSC}_{y}$ & True \\
\hline$X_{1121}$ & $\operatorname{Re} \mathrm{TSC}_{z}$ & False & False & False & $X_{0203}$ & $\mathrm{QSHE}_{y}$ & False \\
\hline$X_{2121}$ & $\operatorname{Im~} \mathrm{TSC}_{z}$ & True & False & False & $X_{3103}$ & $\mathrm{QSHE}_{x}$ & False \\
\hline$X_{1321}$ & $\operatorname{Re} \operatorname{TSC}_{x}$ & False & False & False & $X_{1021}$ & $\operatorname{Re} \operatorname{TSC}_{y}$ & False \\
\hline$X_{2321}$ & $\operatorname{Im} \mathrm{TSC}_{x}$ & True & False & False & $X_{2321}$ & $\operatorname{Im} \mathrm{TSC}_{x}$ & True \\
\hline
\end{tabular}

parameters we have already encountered in the spinless case with charge conservation. There are two valence bond solids, $\operatorname{Re} \operatorname{VBS}(\operatorname{Re} \Delta)$ and $\operatorname{Im} \operatorname{VBS}(\operatorname{Im} \Delta)$. They have maximal symmetry and are invariant under the operation of $C$ conju- gation (9.12). The CDW order parameter $\left(\mu_{\mathrm{s}}\right)$ breaks the SLS. It is mapped into the Néel spin-density wave with quantization axis $z$ under the operation of $C$ conjugation (9.12). The QHE order parameter $(\eta)$ breaks both the SLS 
and TRS symmetries. It is invariant under the operation of $C$ conjugation (9.12).

Second, we break SRS with or without either TRS or SLS while always preserving charge conservation. The breaking of SRS is achieved by choosing a preferred quantization axis, say, $x, y$, or $z$ in SU(2) spin space. Breaking SRS while preserving SLS is achieved with spin-polarized valence-bond ordering in $6=3 \times 2$ different ways, which we abbreviate by Re VBS $, \operatorname{ReVBS}_{y}, \quad \operatorname{Re} \mathrm{VBS}_{z}, \operatorname{Im} \mathrm{VBS}_{x}, \operatorname{Im} \mathrm{VBS}_{y}$, and Im $\mathrm{VBS}_{z}$, in Table II. In doing so TRS is always broken. Breaking SRS and SLS while preserving TRS is achieved through any of the three order parameters for the QSHE introduced by Kane and Mele in Ref. 49, which we abbreviate by $\mathrm{QSHE}_{x}, \mathrm{QSHE}_{y}$, and $\mathrm{QSHE}_{z}$ in Table II. Breaking SRS, SLS, and TRS is achieved through any one of three colinear magnetic order in the form of Néel order, which we abbreviate by $\mathrm{Néel}_{x}, \mathrm{Néel}_{y}$, and $\mathrm{Néel}_{z}$ in Table II.

This brings the number of order parameters that conserve the electronic charge to $16=4+6+3+3$. There are thus 20 $=2+6+6+6$ remaining order parameters that do not conserve the electronic charge.

Third, superconducting order is achieved microscopically by pairing two electrons sitting on different or identical sublattices. In the former case, SLS is preserved. In the latter case, SLS is broken. Pairing of the two electronic spins takes place either in a singlet or in a triplet channel. Antisymmetry under exchange of the two electrons making up a spin-singlet Cooper pair can only be achieved in an even angular momentum channel. On-site pairing is of course associated to vanishing angular momentum so that singlet superconductivity can only be realized when SLS is broken. This only leaves two possible singlet superconducting order parameters that are distinguished by whether they preserve or break TRS. They are denoted Re SSC and Im SSC, respectively. (Real and imaginary parts thus take a different meaning here as for Re VBS and Im VBS.)
Fourth, a triplet superconducting order parameter, which we abbreviate by TSC in Table II, is characterized by a vector $\boldsymbol{d}$ in $\mathrm{SU}(2)$ spin space. This vector can point along any one of the three quantization axis $x, y$, and $z$ in $\mathrm{SU}(2)$ spin space. Moreover, it can either preserve or break TRS for which cases we use the notations Re TSC and Im TSC, respectively, in Table II. (Real and imaginary parts thus take a different meaning here as for Re VBS and Im VBS.) When SLS is preserved by the superconducting order parameter, there are $12=2 \times 2 \times 3$ independent order parameters for a second factor of 2 besides the one for TRS arises since there are two directed nearest-neighbor lattice-bonds connecting nearest-neighbor sites of the two-dimensional lattice. This is abbreviated in Table II by using the index bond $=02,32$ in Re TSC $\mathrm{TSond}_{\text {bo }}, \quad \operatorname{Re} \mathrm{TSC}_{\text {bond } y}, \quad \operatorname{Re~TSC}_{\text {bond } z}, \quad \operatorname{Im} \mathrm{TSC}_{\text {bond } x}$, Im $\mathrm{TSC}_{\mathrm{bond} y}$, and $\mathrm{Im} \mathrm{TSC}_{\mathrm{bond} z}$. Finally, when SLS is broken by the superconducting order parameter, there are $6=2 \times 3$ independent order parameters that we abbreviate by $\operatorname{Re~TSC}_{x}, \quad \operatorname{ReTSC}_{y}, \quad \operatorname{Re} \mathrm{TSC}_{z}, \quad \operatorname{Im} \mathrm{TSC}_{x}, \quad \mathrm{Im} \mathrm{TSC}_{y}$, and Im $\mathrm{TSC}_{z}$, in Table II.

There are $12=4 \times 3$ order parameters that are invariant under the operation of $C$ conjugation (9.12). They can be arranged in four groups of 3 each. Each group of 3 obeys the same algebra. The four groups of 3 are (i) Re VBS, Im VBS, QHE; (ii) Re VBS, Im VBS, $\mathrm{QSHE}_{z}$; (iii) $\mathrm{Re} \mathrm{TSC}_{02 x}$, $\operatorname{Im} \mathrm{TSC}_{32 x}, \operatorname{Im~TSC}_{y}$; and (iv) $\operatorname{Re~TSC}_{02 y}, \operatorname{Im~TSC}_{32 y}$, and $\operatorname{Im} \operatorname{TSC}_{x}$.

The operation of $C$ conjugation (9.12) is a useful tool to identify the possibility of exotic topological effects. For example, we observe that the pair of SSC order parameters Re SSC and Im SSC, studied in the context of graphene, (e.g., Refs. 50-53) are conjugate by $C$ to the Néel order parameters $\mathrm{Néel}_{x}$ and $\mathrm{Néel}_{y}$, respectively. Furthermore, Table II indicates that several triplets of masses that obeys the $\mathrm{SU}(2)$ algebra are related by the operation of $C$ conjugation (9.12). They are

$$
\begin{aligned}
& \left\{\text { Re VBS, Re VBS, CDW } \stackrel{C}{\leftrightarrow}^{C}\left\{\text { Re VBS, Im VBS, } \text { Néel }_{z}\right\},\right.
\end{aligned}
$$

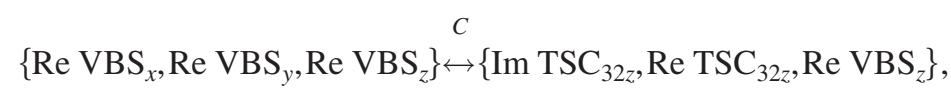

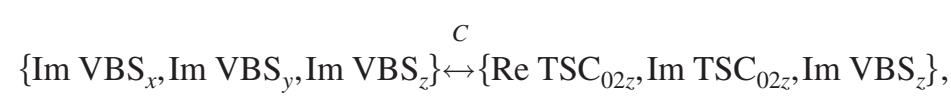

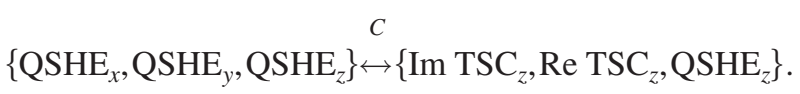

Vortexlike defective textures in any of these mass doublets or meronlike defective textures in any of these mass triplets display fractionalization of some suitably defined quantum numbers.

Finally, the topological property that a band insulator supporting the QSHE carries an odd number of Kramers dou- blets on its edges carries over to the $C$ conjugate TSC. More precisely, the fact that the superconductors with the $\mathrm{Im} \mathrm{TSC}_{z}$ and $\operatorname{Re~TSC}_{z}$ order parameters are examples of $\mathbb{Z}_{2}$ topological triplet superconductors according to Refs. 54-58 is here a mere consequence of their $C$ conjugation with the $\mathrm{QSHE}_{x}$ and $\mathrm{QSHE}_{y}$ order parameters, respectively. 
TABLE III. Enumeration of the 56 distinct five-tuplets of maximally pairwise anticommuting PHS $X_{\mu_{1} \mu_{2} \mu_{3} \mu_{4}}$. The 56 five-tuplets are broken into 28 pairs related by the operation of $C$ conjugation (9.12).

\begin{tabular}{|c|c|}
\hline Five tuplet & Partner five-tuplet by $C$ conjugation \\
\hline$\{$ Re VBS, Im VBS, Re SSC, Im SSC, CDW $\}$ & $\left\{\operatorname{Re}\right.$ VBS, Im VBS, Néel ${ }_{x}$, Néel $_{y}$, Néel $\left._{z}\right\}$ \\
\hline$\left\{\operatorname{Im} V B S, C D W, \operatorname{Re} V_{B S}, \operatorname{Re} \operatorname{VBS}_{y}, \operatorname{Re} \operatorname{VBS}_{z}\right\}$ & $\left\{\operatorname{Im~VBS}, \mathrm{Néel}_{z}, \operatorname{Im~TSC}_{32 z}, \operatorname{Re~TSC}_{32 z}, \operatorname{Re~VBS}_{z}\right\}$ \\
\hline$\left\{\operatorname{Re} V B S, C D W, \operatorname{Im} \operatorname{VBS}_{x}, \operatorname{Im} \operatorname{VBS}_{y}, \operatorname{Im}_{V_{B B S}}\right\}$ & $\left\{\operatorname{Re}\right.$ VBS, $\left.\mathrm{Néel}_{z}, \mathrm{Re}_{\mathrm{TSC}_{02 z}}, \operatorname{Im~TSC}_{02 z}, \operatorname{Im~VBS}_{z}\right\}$ \\
\hline$\left\{\operatorname{Re} \mathrm{SSC}, \mathrm{Im} \mathrm{SSC}, \mathrm{QSHE}_{x}, \mathrm{QSHE}_{y}, \mathrm{QSHE}_{z}\right\}$ & $\left\{\right.$ Néel $_{x}$, Néel $\left._{y}, \operatorname{Im~TSC}_{z}, \operatorname{Re~TSC}_{z}, \mathrm{QSHE}_{z}\right\}$ \\
\hline$\left\{\right.$ Re VBS, Re SSC, Re TSC $\left.{ }_{02 x}, \operatorname{Im}_{\mathrm{TSC}_{02 y}}, \operatorname{Re} \mathrm{TSC}_{02 z}\right\}$ & $\left\{\operatorname{Re}\right.$ VBS, Néel $\left._{x}, \operatorname{Re}_{\mathrm{TSC}_{02 x}}, \operatorname{Im}_{\mathrm{TSC}_{02 x}}, \operatorname{Im~VBS}_{x}\right\}$ \\
\hline$\left\{\right.$ Re VBS, Im SSC, Im TSC $\left.02 x, \operatorname{Re} \mathrm{TSC}_{02 y}, \operatorname{Im~TSC}_{02 z}\right\}$ & $\left\{\operatorname{Re}\right.$ VBS, Néel $\left._{y}, \operatorname{Im~TSC}_{02 y}, \operatorname{Re~TSC}_{02 y}, \operatorname{Im~VBS}_{y}\right\}$ \\
\hline$\left\{\operatorname{Im}\right.$ VBS, Im SSC, Re TSC $\left.32 x, \operatorname{Im~TSC}_{32 y}, \operatorname{Re} \operatorname{TSC}_{32 z}\right\}$ & $\left\{\mathrm{Im} \mathrm{VBS}, \mathrm{Néel}_{y}, \mathrm{Re}_{\mathrm{TSC}_{32 y}}, \operatorname{Im~TSC}_{32 y}, \operatorname{Re}_{\mathrm{VBS}_{y}}\right\}$ \\
\hline$\left\{\operatorname{Im}\right.$ VBS, Re SSC, Im TSC $\left.32 x, \operatorname{Re} \mathrm{TSC}_{32 y}, \operatorname{Im~TSC}_{32 z}\right\}$ & $\left\{\operatorname{Im~VBS}\right.$, Néel $_{x}, \operatorname{Im~TSC}_{32 x}, \operatorname{Re~TSC}_{32 x}, \operatorname{Re}_{\left.\mathrm{VBS}_{x}\right\}}$ \\
\hline$\left\{\mathrm{CDW}, \mathrm{Im} \mathrm{SSC}, \operatorname{Im~TSC}_{x}, \mathrm{Re}_{\mathrm{TSC}_{y}}, \operatorname{Im~TSC}_{z}\right\}$ & $\left\{\right.$ Néel $_{z}$, Néel $\left._{y}, \operatorname{Im~TSC}_{x}, \operatorname{Re~TSC}_{x}, \mathrm{QSHE}_{x}\right\}$ \\
\hline$\left\{\mathrm{CDW}, \mathrm{Re} \mathrm{SSC}, \mathrm{Re} \mathrm{TSC}_{x}, \mathrm{Im} \mathrm{TSC}_{y}, \operatorname{Re} \mathrm{TSC}_{z}\right\}$ & $\left\{\right.$ Néel $_{z}$, Néel $\left._{x}, \operatorname{Re~TSC}_{y}, \operatorname{Im~TSC}_{y}, \mathrm{QSHE}_{y}\right\}$ \\
\hline$\left\{\operatorname{Im~VBS}_{x}, \mathrm{QSHE}_{y}, \operatorname{Im~VBS}_{z}, \operatorname{Re} \mathrm{TSC}_{32 y}, \operatorname{Im~TSC}_{32 y}\right\}$ & $\left\{\operatorname{Re} \mathrm{TSC}_{02 z}, \operatorname{Re} \mathrm{TSC}_{z}, \operatorname{Im}_{\mathrm{VBS}_{z}}, \operatorname{Re} \mathrm{TSC}_{32 x}, \operatorname{Im} \mathrm{TSC}_{32 y}\right\}$ \\
\hline$\left\{\operatorname{Im~VBS}_{x}, \mathrm{QSHE}_{y}, \mathrm{Re}_{\mathrm{VBS}}, \mathrm{Néel}_{x}, \mathrm{QSHE}_{z}\right\}$ & $\left\{\operatorname{Re} \mathrm{TSC}_{02 z}, \operatorname{Re} \mathrm{TSC}_{z}, \operatorname{Im}_{\mathrm{TSC}_{32 z}}, \mathrm{Re} \mathrm{SSC}, \mathrm{QSHE}_{z}\right\}$ \\
\hline$\left\{\operatorname{Im~VBS}_{x}, \operatorname{Re~TSC}_{32 y}, \operatorname{Im~TSC}_{32 z}, \operatorname{Im~TSC}_{02 x}, \operatorname{Im~TSC}_{x}\right\}$ & $\left\{\operatorname{Re~TSC}_{02 z}, \operatorname{Re} \mathrm{TSC}_{32 x}, \operatorname{Re} \mathrm{VBS}_{x}, \operatorname{Im~TSC}_{02 y}, \operatorname{Im~TSC}_{x}\right\}$ \\
\hline$\left\{\operatorname{Im~VBS}_{x}, \operatorname{Re~TSC}_{32 z}, \operatorname{Re} \operatorname{TSC}_{02 x}, \operatorname{Re} \mathrm{TSC}_{x}, \operatorname{Im~TSC}_{32 y}\right\}$ & $\left\{\operatorname{Re}_{\mathrm{TSC}_{02 z}}, \operatorname{Re} \mathrm{VBS}_{y}, \operatorname{Re} \mathrm{TSC}_{02 x}, \operatorname{Re} \mathrm{TSC}_{y}, \operatorname{Im~TSC}_{32 y}\right\}$ \\
\hline$\left\{\operatorname{Im~VBS}_{x}, \operatorname{Re} \mathrm{TSC}_{32 z}, \operatorname{Im~TSC}_{32 z}, \operatorname{Im~VBS}_{y}, \mathrm{QSHE}_{z}\right\}$ & $\left\{\operatorname{Re} \mathrm{TSC}_{02 z}, \operatorname{Re} \mathrm{VBS}_{y}, \operatorname{Re}_{\mathrm{VBS}_{x}}, \operatorname{Im}_{\mathrm{TSC}_{02 z}}, \mathrm{QSHE}_{z}\right\}$ \\
\hline$\left\{\operatorname{Im} \mathrm{VBS}_{x}, \operatorname{Re} \mathrm{TSC}_{x}, \operatorname{Im} \mathrm{TSC}_{x}, \mathrm{CDW}, \operatorname{Re} \mathrm{VBS}_{x}\right\}$ & $\left\{\operatorname{Re} \mathrm{TSC}_{02 z}, \operatorname{Re~TSC}_{y}, \operatorname{Im~TSC}_{x}, \mathrm{Néel}_{z}, \operatorname{Im~TSC}_{32 z}\right\}$ \\
\hline$\left\{\mathrm{QSHE}_{y}, \mathrm{Im} \mathrm{VBS}_{z}, \mathrm{QSHE}_{x}, \mathrm{Re}_{\mathrm{VBS}}, \mathrm{Néel}_{z}\right\}$ & $\left\{\operatorname{Re}_{\mathrm{TSC}}, \operatorname{Im} \mathrm{VBS}_{z}, \operatorname{Im~TSC}_{z}, \operatorname{Re} \mathrm{VBS}_{z}, \mathrm{CDW}\right\}$ \\
\hline$\left\{\mathrm{QSHE}_{y}, \operatorname{Re}_{\mathrm{TSC}} \mathrm{T}_{2 y}, \operatorname{Re} \mathrm{TSC}_{y}, \mathrm{Im} \mathrm{SSC}, \mathrm{Im} \mathrm{TSC}_{32 y}\right\}$ & $\left\{\operatorname{Re} \mathrm{TSC}_{z}, \operatorname{Re} \mathrm{TSC}_{02 y}, \operatorname{Re} \mathrm{TSC}_{x}\right.$, Néel $\left._{y}, \operatorname{Im~TSC}_{32 y}\right\}$ \\
\hline$\left\{\mathrm{QSHE}_{y}, \operatorname{Re} \mathrm{TSC}_{02 y}, \operatorname{Im}_{\mathrm{TSC}_{02 y}}, \operatorname{Re} \mathrm{VBS}_{x}, \operatorname{Re} \mathrm{VBS}_{z}\right\}$ & $\left\{\operatorname{Re~TSC}_{z}, \operatorname{Re} \mathrm{TSC}_{02 y}, \operatorname{Im~TSC}_{02 x}, \operatorname{Im~TSC~}_{32 z}, \operatorname{Re} \mathrm{VBS}_{z}\right\}$ \\
\hline$\left\{\mathrm{QSHE}_{y}, \mathrm{Re}_{\mathrm{TSC}} \mathrm{T}_{2 y}, \operatorname{Im} \mathrm{TSC}_{02 y}, \operatorname{Im} \mathrm{TSC}_{y}, \operatorname{Re} \mathrm{SSC}\right\}$ & $\left\{\operatorname{Re~TSC}_{z}, \operatorname{Re} \mathrm{TSC}_{32 x}, \operatorname{Im~TSC}_{02 x}, \operatorname{Im~TSC}_{y}\right.$, Néel $\left._{x}\right\}$ \\
\hline$\left\{\operatorname{ReVBS}_{y}, \mathrm{Néel}_{y}, \mathrm{QSHE}_{x}, \mathrm{Im} \mathrm{VBS}_{y}, \mathrm{QSHE}_{z}\right\}$ & $\left\{\operatorname{Re~TSC}_{32 z}, \operatorname{Im~SSC}, \operatorname{Im~TSC}_{z}, \operatorname{Im~TSC~}_{02 z}, \mathrm{QSHE}_{z}\right\}$ \\
\hline$\left\{\operatorname{Re~VBS}_{y}, \operatorname{Re} \mathrm{TSC}_{y}, \operatorname{Im~TSC}_{y}, \mathrm{CDW}, \mathrm{Im} \mathrm{VBS}_{y}\right\}$ & $\left\{\operatorname{Re} \mathrm{TSC}_{32 z}, \operatorname{Re~TSC}_{x}, \operatorname{Im~TSC}_{y}\right.$, Néel $\left._{z}, \operatorname{Im~TSC}_{02 z}\right\}$ \\
\hline$\left\{\operatorname{Re~VBS}_{y}, \operatorname{Re} \mathrm{TSC}_{32 y}, \operatorname{Im~TSC}_{y}, \operatorname{Im~TSC}_{02 z}, \operatorname{Im~TSC}_{02 x}\right\}$ & $\left\{\operatorname{Re~TSC}_{32 z}, \operatorname{Re} \mathrm{TSC}_{32 x}, \operatorname{Im} \mathrm{TSC}_{y}, \operatorname{Im} \mathrm{VBS}_{y}, \operatorname{Im~TSC}_{02 y}\right\}$ \\
\hline$\left\{\operatorname{Re} \operatorname{VBS}_{y}, \operatorname{Re} \mathrm{TSC}_{02 x}, \operatorname{Im}_{\mathrm{TSC}_{02 x}}, \mathrm{QSHE}_{x}, \operatorname{ReVBS}_{z}\right\}$ & $\left\{\operatorname{Re~TSC}_{32 z}, \operatorname{Re} \mathrm{TSC}_{02 x}, \operatorname{Im} \mathrm{TSC}_{02 y}, \operatorname{Im} \mathrm{TSC}_{z}, \operatorname{Re}_{\left.\mathrm{VBS}_{z}\right\}}\right\}$ \\
\hline$\left\{\mathrm{Néel}_{y}, \mathrm{Re}_{\mathrm{TSC}} \mathrm{T}_{32}, \operatorname{Im~TSC}_{02 y}, \operatorname{Im~TSC}_{z}, \operatorname{Im~TSC}_{x}\right\}$ & $\left\{\operatorname{Im~SSC}, \operatorname{Re} \mathrm{TSC}_{32 x}, \operatorname{Im}_{\mathrm{TSC}_{02 x}}, \mathrm{QSHE}_{x}, \operatorname{Im~TSC}_{x}\right\}$ \\
\hline$\left\{\operatorname{Im~VBS}_{z}, \operatorname{Re} \mathrm{TSC}_{32 y}, \operatorname{Im}_{\mathrm{TSC}_{02 z}}, \operatorname{Im} \mathrm{TSC}_{z}, \operatorname{Im} \mathrm{TSC}_{32 x}\right\}$ & $\left\{\operatorname{Im~VBS~}_{z}, \operatorname{Re} \mathrm{TSC}_{32 x}, \operatorname{Im} \mathrm{VBS}_{y}, \mathrm{QSHE}_{x}, \operatorname{Im} \mathrm{TSC}_{32 x}\right\}$ \\
\hline$\left\{\operatorname{Re~TSC}_{02 y}, \operatorname{Re} \mathrm{TSC}_{y}, \operatorname{Im~TSC}_{32 z}, \operatorname{Im~TSC}_{32 x}, \operatorname{Im~VBS}_{y}\right\}$ & $\left\{\operatorname{Re~TSC}_{02 y}, \operatorname{Re} \mathrm{TSC}_{x}, \operatorname{Re} \mathrm{VBS}_{x}, \operatorname{Im~TSC}_{32 x}, \operatorname{Im} \mathrm{TSC}_{02 z}\right\}$ \\
\hline$\left\{\operatorname{Re} \operatorname{TSC}_{y}, \operatorname{Re}_{\mathrm{TSC}_{02 x}}, \operatorname{Im~TSC}_{z}, \operatorname{Im}_{\mathrm{TSC}_{32 x}}, \mathrm{Néel}_{x}\right\}$ & $\left\{\operatorname{Re} \mathrm{TSC}_{x}, \operatorname{Re}_{\mathrm{TSC}_{02 x}}, \mathrm{QSHE}_{x}, \operatorname{Im}_{\mathrm{TSC}_{32 x}}, \operatorname{Re} \mathrm{SSC}\right\}$ \\
\hline
\end{tabular}

\section{B. Classification of five tuplets of masses in graphene and $\pi$-flux phases}

Mass matrices that commute pairwise generate competing local order parameters. Conversely, mass matrices that anticommute pairwise generate compatible local order parameters.

All but one PHS masses anticommute with 16 out of the 36 PHS masses. The Haldane mass is unique in that it commutes with all PHS masses.

There are 560 sets of three mutually anticommuting PHS masses. These triplets are generalizations of the triplet of compatible masses $\Delta=\operatorname{Re} \Delta+i \operatorname{Im} \Delta$ and $\mu_{\mathrm{s}}$. Integration over the Dirac fermions in the presence of any one of these mass triplets of mass $m$ in competition with the Haldane mass $\eta$ induces an $\mathrm{O}(3)$ NLSM in $(2+1)$-dimensional space and time with or without a Hopf term for $m>|\eta|$ and $|\eta|>m$, respectively, as was derived in Ref. 15.
There are 280 sets of four mutually anticommuting PHS masses and the maximum number of pairwise anticommuting PHS mass matrices is 5. Out of $\left(\begin{array}{c}36 \\ 5\end{array}\right)=376992$ possibilities, there are 56 distinct five tuplets of compatible PHS mass matrices. They are enumerated in Table III. (If PHS is not imposed, the maximum number of pairwise anticommuting mass matrices in the 64 mass matrices is 7 . There are 288 distinct seven tuplets of compatible mass matrices.)

In the background of each of these five tuplets, integration over the Dirac fermions yields an O(5) NLSM in $(2+1)$-dimensional space and time augmented by a WessZumino-Witten (WZW) term as was derived in Refs. 59 and 60. Defects-driven continuous phase transition between phases of matter unrelated by symmetries (i.e., Landau forbidden) become possible whenever the quantum numbers of the defective order parameters in a given five tuplet are dual in the sense of BF Chern-Simons field theories. ${ }^{61}$ We illustrate this idea with the following examples. 
(a)

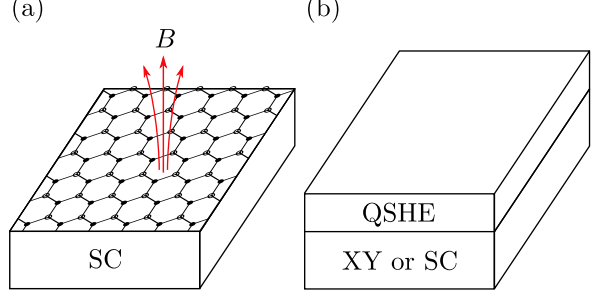

FIG. 7. (Color online) Two setups used to induce topological defects in an order parameter that support fractional quantum numbers.

\section{VBS-SSC-CDW five tuplet}

The five tuplet

\section{$\{$ Re VBS, Im VBS, Re SSC, Im SSC,CDW $\}$}

embeds the triplet made of the CDW and the two VBS order parameters into a five tuplet. ${ }^{61}$ Integration over the fermions yields an $\mathrm{O}(5)$ NLSM augmented by a WZW term for the corresponding five tuplets of bosonic fields $n_{1}, n_{2}, n_{3}, n_{4}$, and $n_{5}$ obeying the constraint that they add in quadrature to unity. The $\mathrm{O}(5)$ symmetry can be broken, either spontaneously or explicitly, down to the $\mathrm{U}(1) \times \mathrm{U}(1)$ subgroup corresponding to holding $\Delta_{\mathrm{CDW}}^{2} \equiv n_{5}^{2}, \Delta_{\mathrm{BDW}}^{2} \equiv n_{1}^{2}+n_{2}^{2}$, and $\Delta_{\mathrm{SSC}}^{2} \equiv n_{3}^{2}+n_{4}^{2}$ fixed (except at the core of topological defects) throughout space and time. The corresponding Goldstone modes are the phases $\theta_{\mathrm{BDW}}$ and $\theta_{\mathrm{SSC}}$. They become charge 2 Higgs fields if the $\mathrm{U}(1) \times \mathrm{U}(1)$ global symmetry they generate is gauged through the introduction of the axial gauge fields $a_{\mathrm{VBS}}^{\mu}$ and the electromagnetic gauge fields $a_{\mathrm{SSC}}^{\mu}$, respectively. Their dynamics is governed by the Anderson-Higgs-Chern-Simons theory (4.13) with the identifications $\theta \rightarrow \theta_{\mathrm{BDW}}, a_{5}^{\mu} \rightarrow a_{\mathrm{VBS}}^{\mu}$, and $a^{\mu} \rightarrow a_{\mathrm{SSC}}^{\mu}-\partial^{\mu} \theta_{\mathrm{SSC}} / 2$. The VBS phase is destroyed when the vortices carried by the conserved topological current $j_{\mathrm{VBS}}^{\mathrm{vr} \mu}=\epsilon^{\mu \nu \rho} \partial_{\nu} \partial_{\rho} \theta_{\mathrm{VBS}} /(2 \pi)$ deconfine. The SSC phase is destroyed when the vortices carried by the conserved topological current $j_{\mathrm{SSC}}^{\mathrm{vrt} \mu}=\epsilon^{\mu \nu \rho} \partial_{\nu} \partial_{\rho} \theta_{\mathrm{SSC}} /(2 \pi)$ deconfine. Because of the BF term in the effective action, the quasiparticles supported by $j_{\mathrm{VBS}}^{\mathrm{vrt} \mu}$ also carry a fraction of the gauge charge of the gauge fields $a_{\mathrm{SSC}}^{\mu}$, while the quasiparticles supported by $j_{\mathrm{SSC}}^{\mathrm{vr} \mu}$ also carry a fraction of the gauge charge of the gauge fields $a_{\mathrm{VBS}}^{\mu}$. Furthermore, both types of quasiparticles are bosons (there is no TRS-breaking Haldane mass). From these two facts follows that deconfinement of one type of quasiparticles implies confinement of the second type of quasiparticles, i.e., a direct transition between the VBS and SSC phases.

An experimental setup to detect exotic quantum numbers related to the five tuplets $(9.14)$ is given in Fig. $7(\mathrm{a}) .{ }^{61} \mathrm{We}$ assume that graphene sits on top of a type-II $s$-wave SC substrate. By the proximity effect, graphene develops a SSC order. The SSC order can coexist with the CDW and VBS orders in graphene according to Eq. (9.14). An applied magnetic field perpendicular to graphene creates an Abrikosov lattice of vortices in the substrate and, by the proximity effect, in graphene. The magnetic flux tubes threading graphene pin axial charges according to Eq. (4.13) (see also Refs. 17 and 61). Increasing the magnetic field so as to de- stroy SSC deconfines the axial charges, i.e., stabilizes the VBS. Conversely, destroying the VBS by the deconfinement of VBS vortices also deconfines the electric charges, i.e., stabilizes the SSC.

\section{VBS-Néel five tuplet}

The operation of $C$ conjugation (9.12) on the five tuplet (9.14) yields the five tuplet

$$
\left\{\text { Re VBS,Im VBS, Néel } x \text {, Néel } y, \text { Néel }_{z}\right\} \text {. }
$$

The triplet of Néel order parameters is here embedded into a five tuplet by adding the doublet of VBS order parameters. ${ }^{59,60,62}$ This five tuplet has been discussed in the context of deconfined quantum criticality of two-dimensional $S=1 / 2$ quantum antiferromagnetic spin models. ${ }^{63-69}$ The five tuplet (9.15) is the only five tuplet supporting the full SU(2) symmetry of the Néel vector. The symmetry analysis of Sec. IX B 1 follows with the identifications $\theta_{\mathrm{VBS}} \rightarrow \theta_{\mathrm{VBS}}$, $\theta_{\mathrm{SSC}} \rightarrow \theta_{\text {Néel }_{x y}}, a_{\mathrm{VBS}}^{\mu} \rightarrow a_{\mathrm{VBS}}^{\mu}, a_{\mathrm{SSC}}^{\mu} \rightarrow a_{\mathrm{Néel}_{x y}}^{\mu}, j_{\mathrm{VBS}}^{\mathrm{vrt} \mu} \rightarrow j_{\mathrm{VBS}}^{\mathrm{vrt} \mu}$, and $j_{\mathrm{SSC}}^{\mathrm{vrt} \mu} \rightarrow j_{\mathrm{Néel}_{x y}}^{\mathrm{vrt} \mu}$.

\section{SSC-QSHE five tuplet}

The five tuplet

$$
\left\{\text { Re SSC,Im SSC, } \mathrm{QSHE}_{x}, \mathrm{QSHE}_{y}, \mathrm{QSHE}_{z}\right\}
$$

embeds the triplet of QSHE order parameters into a five tuplet by adding the two possible SSC order parameters. ${ }^{70-72}$ The symmetry analysis of Sec. IX B 1 follows with the identifications $\quad \theta_{\mathrm{VBS}} \rightarrow \theta_{\mathrm{SSC}}, \quad \theta_{\mathrm{SSC}} \rightarrow \theta_{\mathrm{QSHE}_{x y}}, \quad a_{\mathrm{VBS}}^{\mu} \rightarrow a_{\mathrm{SSC}}^{\mu}$, $a_{\mathrm{SSC}}^{\mu} \rightarrow a_{\mathrm{QSHE}_{x y}}^{\mu}, j_{\mathrm{VBS}}^{\mathrm{vrt} \mu} \rightarrow j_{\mathrm{SSC}}^{\mathrm{vrt} \mu}$, and $j_{\mathrm{SSC}}^{\mathrm{vrt} \mu} \rightarrow j_{\mathrm{QSHE}_{x y}}^{\mathrm{vrt} \mu}$.

An experimental setup to detect exotic quantum numbers related to the five tuplet (9.16) is given in Fig. 7(b). We bring in contact a (three-dimensional) bulk type-II SSC with a material displaying the QSHE. Instead of graphene for which the spin-orbit coupling is very small, $\mathrm{HgTe} /(\mathrm{Hg}, \mathrm{Cd}) \mathrm{Te}$ semiconductor quantum wells are suitable. ${ }^{73-75}$ Any SSC vortex in the substrate induces by proximity effect an " $S_{z}$ spin charge" in the device supporting the QSHE, while any $S_{z}$ "spin flux" in the device supporting the QSHE induces an electric charge.

\section{XY-Néel-TSC-QSHE five tuplet}

The operation of $C$ conjugation (9.12) on the five tuplet (9.16) yields the five tuplet ${ }^{71,72}$

$$
\left\{\mathrm{Néel}_{x}, \mathrm{Néel}_{y}, \mathrm{Im} \mathrm{TSC}_{z}, \mathrm{Re} \mathrm{TSC}_{z}, \mathrm{QSHE}_{z}\right\} \text {. }
$$

By rotating $\mathrm{SU}(2)$ spin quantization axis, i.e., by cyclic permutation of the indices $x, y$, and $z$, we also get the five tuplets

$$
\left\{\mathrm{Néel}_{y}, \mathrm{Néel}_{z}, \operatorname{Im} \mathrm{TSC}_{x}, \mathrm{Re}_{\mathrm{TSC}}, \mathrm{QSHE}_{x}\right\}
$$

and

$$
\left\{\text { Néel }_{z}, \text { Néel }_{x}, \operatorname{Im~TSC}_{y}, \operatorname{Re~TSC}_{y}, \mathrm{QSHE}_{y}\right\} .
$$

These five tuplets describe SLS- and SRS-breaking order parameters consisting of an easy plane antiferromagnetic 
order parameter coexisting with the QSHE and TSC order parameters. The symmetry analysis of Sec. IX B 1 follows with the identifications $\quad \theta_{\mathrm{VBS}} \rightarrow \theta_{\mathrm{Néel}_{x y}}, \quad \theta_{\mathrm{SSC}} \rightarrow \theta_{\mathrm{TSC}_{z}}$, $a_{\mathrm{VBS}}^{\mu} \rightarrow a_{\mathrm{Néel}_{x y}}^{\mu}, a_{\mathrm{SSC}}^{\mu} \rightarrow a_{\mathrm{TSC}_{z}}^{\mu}, j_{\mathrm{VBS}}^{\mathrm{vrt} \mu} \rightarrow j_{\mathrm{Néel}_{x y}}^{\mathrm{vrt} \mu}$, and $j_{\mathrm{SSC}}^{\mathrm{vrt} \mu} \rightarrow j_{\mathrm{TSC}_{z}}^{\mathrm{vrt} \mu^{z}}$, say.

An experimental setup to detect exotic quantum numbers related to the five tuplet $(9.17 \mathrm{a}),(9.17 \mathrm{~b})$, and $(9.17 \mathrm{c})$ is also given in Fig. 7(b). Any defect in the bulk $X Y$ antiferromagnet, i.e., a magnetic vortex, induces a localized midgap state that carries a fraction of the electric charge carried by the phase of the TSC in the band insulator supporting the QSHE. Any TSC vortex induces an $S_{z}$ spin charge in the device supporting the QSHE. [A related fractional (electrical) charge is discussed at the helical edges of the QSHE. $\left.{ }^{76}\right]$

\section{DISCUSSION}

Motivated by the interplay between charge-density $\left(\mu_{\mathrm{s}}\right)$, bond-density $\left(\Delta=|\Delta| e^{-i \theta}\right)$, and integer quantum Hall $(\eta)$ instabilities in graphenelike two-dimensional electronic systems, we have computed the fractional charge and fractional statistics of both screened and unscreened quasiparticles.

At the microscopic level, screened quasiparticles are here the linear superpositions of two bond-density waves ( $\Delta$ and $\boldsymbol{a}_{5}$ ), each of which carry a point defect. Unscreened quasiparticles are defects in one type $(\Delta)$ of bond-density wave.

In the long-wavelength and low-energy limit and after integrating out the fermions, the quantum dynamics of screened quasiparticles is controlled by the effective theory (4.13) of the Anderson-Higgs-Chern-Simons type involving three fields. There are two U(1) gauge fields and one phase field.

The first gauge field $a_{\mu}$ is responsible for the conservation of the total fermion number. The second gauge field $a_{5 \mu}$ is responsible for the conservation of a relative fermion number, i.e., the difference in the fermion number located at the two valleys of graphene say, and is thus called an axial gauge field. The phase field $\theta=-\arg \Delta$ originates microscopically from the fact that bond distortions include atomic displacements away from the crystalline order that are parametrized by continuous angular degrees of freedom.

Screened quasiparticles are not yet explicitly manifest in the field theory (4.13). They appear as point particles with the conserved topological current $\bar{j}_{\mathrm{vrt}}^{\mu}=(2 \pi)^{-1} \epsilon^{\mu \nu \lambda} \partial_{\nu} \partial_{\lambda} \theta$ that carries no axial gauge charge, once a duality transformation has been performed. The Lagrangian dual to Lagrangian (4.13) can be presented as a Chern-Simons theory for four gauge fields whose $K$ matrix $^{77}$ is four dimensional and couple through a four-dimensional charge vector to the vortex current. Because the $K$ matrix has a vanishing eigenvalue, ${ }^{77}$ this dual theory is not a topological theory, say, such as a BF Chern-Simons theory. ${ }^{44,45}$ The vanishing eigenvalue of the $K$ matrix signals the existence of lowenergy excitations, the screened quasiparticles. Their fractional charges $Q$ and statistical angle $\Theta$ can then be calculated and are presented in the phase diagram of Fig. 1.

When the $\mathrm{U}(1) \times \mathrm{U}(1)$ local gauge symmetry holds, i.e., for screened quasiparticles that represent vortices in the phase field $\theta$ whose axial charges are dynamically screened by axial gauge half fluxes in $a_{5 \mu}$, the fractional charge $Q$ and the fractional statistical angle $\Theta$ in Fig. 1 are complementary. One is nonvanishing if and only if the other vanishes. Moreover, $Q$ and $\Theta$ are universal in the fully gaped phases for which they are nonvanishing and given by a rational number in some units.

When the $\mathrm{U}(1) \times \mathrm{U}(1)$ local gauge symmetry is broken, i.e., for unscreened quasiparticles that represent vortices in the phase field $\theta$ without the attachment of axial gauge half fluxes, the fractional statistical angle $\Theta$ is nonvanishing everywhere in Fig. 1 with a discontinuous jump at $m \equiv \sqrt{\mu_{\mathrm{s}}^{2}+|\Delta|^{2}}=|\eta|$ and a nonuniversal dependence on the ratios $\mu_{\mathrm{s}} / m$ and $\eta / m$. The fractional charge $Q$ is only nonvanishing when $|\eta|<m$ where it is also nonuniversal.

Comparing the values of $\Theta$ in Fig. 1 calculated from field theory with a numerical evaluation of $\Theta$ for an underlying microscopic (lattice) model is difficult for two reasons. Defects in the phase $\theta$ have a characteristic size of the order of $1 / m$ for lattice models, i.e., they bind a fermionic charge through midgap states. The profile of defects in the axial gauge fields $a_{5 \mu}$ is power law, i.e., they bind a fermionic charge through threshold continuum states. Thus, the linear extend of any lattice model must be much larger than $1 / \mathrm{m}$ for any reliable numerical calculation of $\Theta$. On the one hand, if we impose the $\mathrm{U}(1) \times \mathrm{U}(1)$ local gauge invariance at the lattice level, the system sizes accessible to a numerical computation of $\Theta$ are, at best, of the order $1 / m$, i.e., too small for a comparison with field theory. On the other hand, if the $\mathrm{U}(1) \times \mathrm{U}(1)$ local gauge invariance does not hold at the lattice level, say, after performing a mean-field approximation for which the accessible system sizes are sufficient to measure $Q$ with the help of a static probe such as the spectral asymmetry, then the values of $Q$ and $\Theta$ are not universal anymore. To put it differently, the values of $Q$ and $\Theta$ measured dynamically depend sensitively on the dynamical rules used. But these dynamical rules are model dependent when they are not fixed by imposing the local axial gauge symmetry.

The fractional charge $Q$ or the statistical angle $\Theta$ in the phase diagram of Fig. 1 disagree with the results of Refs. 15, 17, and 18. Although the charge assignment in Ref. 15 agrees with that in Fig. 1 the statistical angle is ascribed the value $\Theta=\operatorname{sgn}(\eta) \pi / 4$ whenever $\eta \neq 0 .{ }^{78}$ However, the statistical angle $\Theta$ is nonvanishing if and only if the Hopf term is present in the $\mathrm{O}(3)$ nonlinear-sigma model derived in Ref. 15 , i.e., if and only if $|\eta|>m$, in which case full agreement with the charge and statistical angle assignments of Fig. 1 is recovered.

Seradjeh and Franz in Ref. 17 have computed the fractional charge $Q$ and fractional statistics $\Theta$ of dynamical defects in $\Theta$ and $\boldsymbol{a}_{5}$ for the field theory (3.1a), (3.1b), (3.1c), and (3.1d) when $\mu_{\mathrm{s}}=\eta=0$. Their analysis has been repeated by Milovanovic in Ref. 18. They found the assignments $Q= \pm 1 / 2$ and $\Theta= \pm \pi$. Their semion statistics contradicts our result $\Theta=0$ in Fig. 1. This discrepancy can be traced to the fact that Seradjeh and Franz used a singular chiral U(1) gauge transformation with the Pauli-Villars regularization to derive an effective action different than Eq. (4.13). As we show in Appendix D the effective action used by Seradjeh and Franz, when suitably generalized to the case $\mu_{\mathrm{s}} \neq \eta=0$, 
fails to reproduce the fractional charge (5.7) of quasiparticles in the presence of a flux in $\boldsymbol{a}_{5}$ gauge field. Explicitly, it follows from Eq. (9) of their paper in Ref. 17 that the fractional charge in the case when the mass vortex is accompanied by an axial half-flux, enforcing the screening condition $a_{5 \kappa}-\frac{1}{2} \partial_{\kappa} \theta=0$, is $Q=0$. However, the fractional charge $Q=1 / 2$ [see Eq. (5.7)] of screened quasiparticles is a result established from direct (static) numerical computation of $Q$ on a suitable lattice regularization of the field theory (3.1a), (3.1b), (3.1c), and (3.1d).

The charge-density $\left(\mu_{\mathrm{s}}\right)$, bond-density $(\Delta)$, and integer quantum Hall $(\eta)$ instabilities are the only instabilities compatible with the electron-number conservation and $\mathrm{SU}(2)$ spin-rotation symmetry (these are, naturally, also the only four possible instabilities for the spinless case). However, there can also be superconducting instabilities or, if the electron spin is accounted for, magnetic instabilities. We have performed a systematic classification of all instabilities for the 16-dimensional free Dirac Hamiltonian induced by local order parameters that respect the Bogoliubov-de-Gennes particle-hole symmetry. We have found that the order parameter for the integer quantum Hall effect (Haldane mass $\eta$ ) is unique for it competes with all other instabilities. We have also found that the largest number of coexisting order parameters is 5 and enumerated all the corresponding five tuplets of masses. Each of these five tuplet can be thought of as a generalization of the three tuplet $\left(\mu_{\mathrm{s}}, \operatorname{Re} \Delta, \operatorname{Im} \Delta\right)$ that supports quasiparticles with fractional quantum numbers. These five tuplets provide a rich playground for Landau-forbidden continuous phase transitions. Any U(1) order parameter in a five tuplet can be assigned a conserved charge and supports topological defects in the form of vortices. A pair of $U(1)$ order parameters in a five tuplet is said to be dual if the vortices of one order parameter binds the charge of the other order parameter and vice versa. A continuous phase transition can then connect directly the two dual U(1) ordered phases through a confining-deconfining transition of their vortices.

\section{ACKNOWLEDGMENTS}

We thank M. Franz and B. Seradjeh for useful discussions. This work is supported in part by the DOE Grant No. DE-FG02-06ER46316 (C.-Y. H. and C. C.). C.M. acknowledges the kind hospitality of the Isaac Newton Institute, Cambridge and RIKEN. We thank the Condensed Matter Theory Visitor's Program at Boston University for support. S.R. thanks the Center for Condensed Matter Theory at University of California, Berkeley for its support. S.R. thanks P. Ghaemi, D.-H. Lee, and A. Vishwanath for useful discussions.

\section{APPENDIX A: CALCULATIONS OF THE COEFFICIENTS $C_{11}^{(0)}, C_{00}^{(1)}, C_{33}^{(1)}, C_{11}^{(1)}$, AND $C_{03}^{(1)}$}

Let $B_{\mu}$ be a four-dimensional representation of an element of the Lie Algebra $u(2)$ generated by the unit $4 \times 4$ matrix and the $4 \times 4$ matrices $\Sigma_{\mathrm{a}}=\left(\Sigma_{1}, \Sigma_{2}, \Sigma_{3}\right)$ whereby

$$
\left[\Sigma_{\mathrm{a}}, \Sigma_{\mathrm{b}}\right]=i \epsilon_{\mathrm{abc}} \Sigma_{\mathrm{c}}, \quad\left\{\Sigma_{\mathrm{a}}, \Sigma_{\mathrm{b}}\right\}=2 \delta_{\mathrm{ab}}
$$

In this appendix, we are going to integrate the Grassmann fields $\bar{\chi}$ and $\chi$ in the partition function,

$$
\begin{gathered}
Z:=\int \mathcal{D}[\bar{\chi}, \chi] e^{i S}, \\
S:=\int d x^{0} d x^{1} d x^{2} \mathcal{L}, \\
\mathcal{L}:=\bar{\chi}\left(i \not \partial_{\mu}+\not B-m \Sigma_{3}-\eta\right) \chi .
\end{gathered}
$$

Here, the Feynman slash notation

$$
\phi \equiv \Gamma^{\mu} \partial_{\mu}, \quad \not B \equiv \Gamma^{\mu} B_{\mu}
$$

is used when contracting three vectors with the $4 \times 4$ matrices $\Gamma_{\mu}=\left(\Gamma_{0}, \Gamma_{1}, \Gamma_{2}\right)$ that realize a four-dimensional representation of the algebra

$$
\left\{\Gamma_{\mu}, \Gamma_{\nu}\right\}=2 g_{\mu \nu}, \quad g_{\mu \nu}=\operatorname{diag}(1,-1,-1),
$$

while they commute with $\Sigma_{\mathrm{a}}=\left(\Sigma_{1}, \Sigma_{2}, \Sigma_{3}\right)$,

$$
\left[\Gamma_{\mu}, \Sigma_{\mathrm{a}}\right]=0 \text {. }
$$

We shall work in momentum space. To this end, we introduce the Fourier transforms,

$$
\begin{gathered}
\bar{\chi}(x)=\int_{k} e^{-i k \cdot x} \bar{\chi}(k), \\
\chi(x)=\int_{k} e^{i k \cdot x} \chi(k), \\
B_{\mu}(x)=\int_{k} e^{i k \cdot x} B_{\mu}(k),
\end{gathered}
$$

whereby the notations

$$
\begin{gathered}
k \cdot x \equiv k^{\mu} x_{\mu}=k^{\mu} g_{\mu \nu} x^{\mu}, \\
k^{2} \equiv k^{\mu} k_{\mu}=k^{\mu} g_{\mu \nu} k^{\mu}, \\
\int_{k} \equiv \int \frac{d k^{0} d k^{1} d k^{2}}{(2 \pi)^{3}}
\end{gathered}
$$

will be used. The action and Lagrangian in Eq. (A2) are represented in momentum space by

$$
\begin{gathered}
S=\int_{k_{1}, k_{2}} \mathcal{L}, \\
\mathcal{L}=\bar{\chi}\left(k_{1}\right)\left[G_{0}^{-1}\left(k_{1}\right) \delta\left(k_{1}-k_{2}\right)+\mathbb{B}\left(k_{1}-k_{2}\right)\right] \chi\left(k_{2}\right) .
\end{gathered}
$$

The free propagator, here defined by 


$$
\begin{aligned}
G_{0}(k) & :=-\frac{1}{k+\eta+m \Sigma_{3}} \\
& =-\frac{k-\eta-m \Sigma_{3}}{k^{2}-\eta^{2}-m^{2}-2 \eta m \Sigma_{3}} \\
& =-\frac{\left(k-\eta-m \Sigma_{3}\right)\left(k^{2}-\eta^{2}-m^{2}+2 \eta m \Sigma_{3}\right)}{\left(k^{2}-(\eta-m)^{2}\right)\left(k^{2}-(\eta+m)^{2}\right)}
\end{aligned}
$$

(A6a)

can be decomposed into the sum of the unit $4 \times 4$ matrix weighted by the factor $P(k)$ and the $4 \times 4$ matrix $\Sigma_{3}$ weighted by the factor $Q(k)$,

$$
\begin{gathered}
G_{0}(k)=P(k)+Q(k) \Sigma_{3}, \\
P(k)=-\frac{k\left(k^{2}-\eta^{2}-m^{2}\right)-\eta\left(k^{2}-\eta^{2}+m^{2}\right)}{\left(k^{2}-(\eta-m)^{2}\right)\left(k^{2}-(\eta+m)^{2}\right)}, \\
Q(k)=-\frac{2 \eta m k-m\left(k^{2}+\eta^{2}-m^{2}\right)}{\left(k^{2}-(\eta-m)^{2}\right)\left(k^{2}-(\eta+m)^{2}\right)} .
\end{gathered}
$$

The induced effective action for the background $\boldsymbol{B}$ is defined by

$$
\begin{gathered}
\exp \left(i S_{\mathrm{eff}}[B]\right) \propto \int \mathcal{D}[\bar{\chi}, \chi] \exp (i S[B]), \\
S_{\mathrm{eff}}[B]:=i \sum_{n=1}^{\infty} \frac{(-1)^{n}}{n} \operatorname{Tr}\left(G_{0} \boldsymbol{B}\right)^{n} \equiv i \sum_{n=1}^{\infty} S_{\mathrm{eff}}^{(n)}[B],
\end{gathered}
$$

where it is understood that the Grassmann integration is performed in a way that preserves the local $\mathrm{U}(1) \times \mathrm{U}(1)$ gauge symmetry (4.3b). The effective action in Eq. (4.10a) and (4.10b) with the coefficients from Table I follows by combining the local $\mathrm{U}(1) \times \mathrm{U}(1)$ gauge symmetry $(4.3 \mathrm{~b})$ with the loop expansion (A7) up to the order $n=2$,

$$
\begin{gathered}
S_{\mathrm{eff}}[B] \approx i S_{\mathrm{eff}}^{(1)}[B]+i S_{\mathrm{eff}}^{(2)}[B]+\cdots, \\
S_{\mathrm{eff}}^{(1)}[B]=\int_{k} \operatorname{tr}\left[G_{0}(k) \boldsymbol{B}(0)\right], \\
S_{\mathrm{eff}}^{(2)}[B]=\frac{1}{2} \int_{k, q} \operatorname{tr}\left[G_{0}(k) \boldsymbol{B}(q) G_{0}(k-q) \boldsymbol{B}(-q)\right] .
\end{gathered}
$$

One verifies by explicit calculation that

$$
S_{\mathrm{eff}}^{(1)}[B]=0 \text {. }
$$

To proceed with the evaluation of $S_{\mathrm{eff}}^{(2)}[\mathbb{B}]$, we note that the algebra (A1), (A3b), and (A3c) can always be realized with the choice

$$
\Gamma_{\mu}=\gamma_{\mu} \otimes \mathbb{1}_{\sigma}, \quad \Sigma_{\mathrm{a}}=\mathbb{1}_{\gamma} \otimes \sigma_{\mathrm{a}}
$$

where $\gamma_{\mu}=\left(\gamma_{0}, \gamma_{1}, \gamma_{2}\right)$ and $\sigma_{\mathrm{a}}=\left(\sigma_{1}, \sigma_{2}, \sigma_{3}\right)$ realize twodimensional representations of algebras (A3b) and (A1), respectively. With this choice, it is obvious that a single trace over the $4 \times 4$ matrices spanned by the unit $4 \times 4$ matrix,
$\Gamma_{\mu}=\left(\Gamma_{0}, \Gamma_{1}, \Gamma_{2}\right)$, and $\Sigma_{\mathrm{a}}=\left(\Sigma_{1}, \Sigma_{2}, \Sigma_{3}\right)$ factorizes into the product over two traces; one trace over the $2 \times 2$ matrices spanned by the unit matrix $1_{\gamma}$ and $\gamma_{\mu}=\left(\gamma_{0}, \gamma_{1}, \gamma_{2}\right)$ and one trace over the $2 \times 2$ matrices spanned by the unit matrix $1_{\sigma}$ and $\sigma_{\mathrm{a}}=\left(\sigma_{1}, \sigma_{2}, \sigma_{3}\right)$. It then follows that

$$
\begin{aligned}
S_{\mathrm{eff}}^{(2)}[B]= & \frac{1}{2} \int_{q}[P P(q)]^{\nu \kappa} \operatorname{tr}_{\sigma}\left[B_{\nu}(q) B_{\kappa}(-q)\right] \\
& +\frac{1}{2} \int_{q}[P Q(q)]^{\nu \kappa} \operatorname{tr}_{\sigma}\left[B_{\nu}(q) \sigma_{3} B_{\kappa}(-q)\right] \\
& +\frac{1}{2} \int_{q}[Q P(q)]^{\nu \kappa} \operatorname{tr}_{\sigma}\left[\sigma_{3} B_{\nu}(q) B_{\kappa}(-q)\right] \\
& +\frac{1}{2} \int_{q}[Q Q(q)]^{\nu \kappa} \operatorname{tr}_{\sigma}\left[\sigma_{3} B_{\nu}(q) \sigma_{3} B_{\kappa}(-q)\right],
\end{aligned}
$$

where $[H K(q)]^{\nu \kappa}, H$ and $K$ being $P$ or $Q$, is defined as

$$
[H K(q)]^{\nu \kappa}:=\int_{k} \operatorname{tr}_{\gamma}\left[H(k) \gamma^{\nu} K(k-q) \gamma^{\kappa}\right],
$$

and with the understanding that

$$
B_{\mu}=b_{\mu}^{\mathrm{a}} \sigma^{\mathrm{a}}, \quad k=\gamma^{\mu} k_{\mu} .
$$

If the integrals (A11b) are regularized so as to preserve the Lorentz covariance, then they must be of the form

$$
[H K(q)]^{\nu \kappa}=g^{\nu \kappa}[H K]^{(0)}+i \epsilon^{\nu \rho \kappa} q_{\rho}[H K]^{(1)}+\cdots,
$$

to linear order in $q$. Furthermore, imposing a regularization of the integrals (A11b) that preserves the local $\mathrm{U}(1) \times \mathrm{U}(1)$ gauge symmetry $(4.3 \mathrm{~b})$ demands that the coefficients

$$
\begin{gathered}
C_{00}^{(0)}=C_{33}^{(0)}:=[P P]^{(0)}+[Q Q]^{(0)}=0, \\
C_{03}^{(0)}:=2\left([P Q]^{(0)}+[Q P]^{(0)}\right)=0 .
\end{gathered}
$$

This gives the effective Lagrangian

$$
\begin{aligned}
\mathcal{L}_{\text {eff }}^{(2)}= & C_{11}^{(0)}\left(b^{1 \rho} b_{\rho}^{1}+b^{2 \rho} b_{\rho}^{2}\right)+C_{00}^{(1)} \epsilon^{\nu \rho \kappa} b_{\nu}^{0} \partial_{\rho} b_{\kappa}^{0}+C_{33}^{(1)} \epsilon^{\nu \rho \kappa} b_{\nu}^{3} \partial_{\rho} b_{\kappa}^{3} \\
& +C_{11}^{(1)} \epsilon^{\nu \rho \kappa}\left(b_{\nu}^{1} \partial_{\rho} b_{\kappa}^{1}+b_{\nu}^{2} \partial_{\rho} b_{\kappa}^{2}+\cdots\right)+C_{03}^{(1)} \epsilon^{\nu \rho \kappa} b_{\nu}^{0} \partial_{\rho} b_{\kappa}^{3}
\end{aligned}
$$

with the coefficients

$$
\begin{gathered}
C_{11}^{(0)}:=[P P]^{(0)}-[Q Q]^{(0)}, \\
C_{00}^{(1)}=C_{33}^{(1)}:=[P P]^{(1)}+[Q Q]^{(1)}, \\
C_{11}^{(1)}:=[P P]^{(1)}-[Q Q]^{(1)}, \\
C_{03}^{(1)}:=2\left([P Q]^{(1)}+[Q P]^{(1)}\right) .
\end{gathered}
$$

Coefficients (A15) are evaluated by performing a Wick rotation to the Euclidean metric with the rules

$$
t \rightarrow-i \tau, \quad \boldsymbol{r} \rightarrow \boldsymbol{r}, \quad \gamma^{0} \rightarrow \gamma^{0}, \quad \boldsymbol{\gamma} \rightarrow i \boldsymbol{\gamma}
$$




$$
b_{0}^{\mathrm{a}} \rightarrow i b_{0}^{\mathrm{a}}, \quad \boldsymbol{b}^{\mathrm{a}} \rightarrow-\boldsymbol{b}^{\mathrm{a}}, \quad a=1,2,3 .
$$

Under these rules

$$
g^{\mu \nu} \rightarrow-\delta^{\mu \nu}, \quad \epsilon^{\mu \nu \lambda} \rightarrow i \epsilon^{\mu \nu \lambda},
$$

while the scalar functions $P(k)$ and $Q(k)$ in propagator (A6b) take the form

$$
P(k)=[R(k) k+S(k)] \otimes 1_{\sigma}, \quad Q(k)=[T(k) k+U(k)] \otimes 1_{\sigma},
$$

with $m_{ \pm}:=m \pm \eta$ and

$$
\begin{gathered}
R(k)=\frac{i\left(k^{2}+\frac{m_{+}^{2}+m_{-}^{2}}{2}\right)}{\left(k^{2}+m_{+}^{2}\right)\left(k^{2}+m_{-}^{2}\right)}, \quad S(k)=\frac{-\eta\left(k^{2}-m_{+} m_{-}\right)}{\left(k^{2}+m_{+}^{2}\right)\left(k^{2}+m_{-}^{2}\right)}, \\
T(k)=\frac{-2 \eta m i}{\left(k^{2}+m_{+}^{2}\right)\left(k^{2}+m_{-}^{2}\right)}, \quad U(k)=-m\left(k^{2}+m_{+} m_{-}\right) .
\end{gathered}
$$

Their small $q$ expansion are

$$
\begin{aligned}
& R(k-q)=R(k)+(k \cdot q) R^{(1)}(k), \\
& S(k-q)=S(k)+(k \cdot q) S^{(1)}(k), \\
& T(k-q)=T(k)+(k \cdot q) T^{(1)}(k), \\
& U(k-q)=U(k)+(k \cdot q) U^{(1)}(k),
\end{aligned}
$$

where

$$
\begin{aligned}
R^{(1)}(k)= & \frac{2 i}{\left(k^{2}+m_{+}^{2}\right)^{2}\left(k^{2}+m_{-}^{2}\right)^{2}} \\
& \times\left(k^{4}+\left(m_{+}^{2}+m_{-}^{2}\right) k^{2}-m_{+}^{2} m_{-}^{2}+\frac{\left(m_{+}^{2}+m_{-}^{2}\right)^{2}}{2}\right), \\
S^{(1)}(k)= & \frac{-2 \eta}{\left(k^{2}+m_{+}^{2}\right)^{2}\left(k^{2}+m_{-}^{2}\right)^{2}} \\
& \times\left[k^{4}-2 k^{2} m_{+} m_{-}-m_{+}^{2} m_{-}^{2}-m_{+} m_{-}\left(m_{+}^{2}+m_{-}^{2}\right)\right], \\
T^{(1)}(k) & =\frac{-4 \eta m i}{\left(k^{2}+m_{+}^{2}\right)^{2}\left(k^{2}+m_{-}^{2}\right)^{2}}\left[2 k^{2}+\left(m_{+}^{2}+m_{-}^{2}\right)\right], \\
U^{(1)}(k)= & \frac{-2 m}{\left(k^{2}+m_{+}^{2}\right)^{2}\left(k^{2}+m_{-}^{2}\right)^{2}} \\
& \times\left[k^{4}+2 k^{2} m_{+} m_{-}-m_{+}^{2} m_{-}^{2}+m_{+} m_{-}\left(m_{+}^{2}+m_{-}^{2}\right)\right] .
\end{aligned}
$$

At last, coefficients (A15) follow from

$$
C_{11}^{(0)}=-\left([P P]^{(0)}-[Q Q]^{(0)}\right),
$$

$$
C_{00}^{(1)}=C_{33}^{(1)}=-i\left([P P]^{(1)}+[Q Q]^{(1)}\right),
$$

$$
C_{11}^{(1)}=-i\left([P P]^{(1)}-[Q Q]^{(1)}\right),
$$

$$
C_{03}^{(1)}=-2 i\left([P Q]^{(1)}+[Q P]^{(1)}\right),
$$

with

$$
\begin{gathered}
{[P P]^{(0)}=\frac{4 \pi}{(2 \pi)^{3}}\left(-\frac{2}{3} I_{R R}+2 \widetilde{I}_{S S}\right)} \\
{[P P]^{(1)}=\frac{8 \pi}{(2 \pi)^{3}}\left(-\frac{1}{3} I_{R S^{(1)}}-\tilde{I}_{S R}+\frac{1}{3} I_{S R^{(1)}}\right)} \\
{[P Q]^{(0)}=\frac{4 \pi}{(2 \pi)^{3}}\left(-\frac{2}{3} I_{R T}+2 \tilde{I}_{S U}\right)} \\
{[P Q]^{(1)}=\frac{8 \pi}{(2 \pi)^{3}}\left(-\frac{1}{3} I_{R U^{(1)}}-\widetilde{I}_{S T}+\frac{1}{3} I_{S T^{(1)}}\right)}
\end{gathered}
$$

$$
\begin{gathered}
{[Q P]^{(0)}=\frac{4 \pi}{(2 \pi)^{3}}\left(-\frac{2}{3} I_{T R}+2 \widetilde{I}_{U S}\right)} \\
{[Q P]^{(1)}=\frac{8 \pi}{(2 \pi)^{3}}\left(-\frac{1}{3} I_{T S^{(1)}}-\widetilde{I}_{U R}+\frac{1}{3} I_{U R^{(1)}}\right),} \\
{[Q Q]^{(0)}=\frac{4 \pi}{(2 \pi)^{3}}\left(-\frac{2}{3} I_{T T}+2 \widetilde{I}_{U U}\right)}
\end{gathered}
$$$$
[Q Q]^{(1)}=\frac{8 \pi}{(2 \pi)^{3}}\left(-\frac{1}{3} I_{T U^{(1)}}-\tilde{I}_{U T}+\frac{1}{3} I_{U T^{(1)}}\right) .
$$

Here, the integrals

$$
\begin{gathered}
I_{X Y} \equiv \int_{0}^{\infty} d k k^{4} X(k) Y(k), \\
\tilde{I}_{X Y} \equiv \int_{0}^{\infty} d k k^{2} X(k) Y(k),
\end{gathered}
$$

with $X(k)$ and $Y(k)$ denoting $R(k), R^{(1)}(k), S(k), S^{(1)}(k), T(k)$, $T^{(1)}(k), U(k)$, or $U^{(1)}(k)$ need to be regularized in a way that preserves the local $\mathrm{U}(1) \times \mathrm{U}(1)$ gauge symmetry (4.3b) and the Lorentz covariance. The brute force method consisting in imposing the ultraviolet cutoff $\Lambda$ in the integrals (A20f) and ignoring all the terms linear in $\Lambda$ delivers the coefficients from Table I.

\section{APPENDIX B: DUALITY AND STATISTICS IN THE QUANTUM $X Y$ MODEL WITH A CHERN-SIMONS TERM}

The presentation of the effective action (4.13) with the help of Table I is not optimal for the purpose of extracting 
the statistical angle $\Theta$ acquired by the pairwise exchange of unit vortices from Sec. V. Needed is a conserved vortex current that accounts for the local vortex density and the local vortex current generated by the physical process involving the exchange of two vortices. This vortex current can be non-vanishing anywhere in the phase diagram in Fig. 1. Thus, an optimal presentation of the effective action (4.13) should include this vortex current. This can be achieved by taking advantage of the duality between the quantum $X Y$ model and compact quantum electrodynamics in $(2+1)$-dimensional space and time,${ }^{79-83}$ which we now briefly adapt for our purpose.

\section{Duality}

We begin by defining the partition function for the quantum $X Y$ model in $(2+1)$-dimensional space and time with an additional Chern-Simons term,

$$
\begin{gathered}
Z_{X Y}^{C S}:=\int \mathcal{D}[\theta] \exp \left(i \int d^{3} x\left(\mathcal{L}_{X Y}[\theta]+\mathcal{L}_{C S}[\theta]\right)\right), \\
\mathcal{L}_{X Y}[\theta]:=\frac{K}{2}\left(\partial_{\mu} \theta\right)\left(\partial^{\mu} \theta\right), \\
\mathcal{L}_{C S}[\theta]:=\frac{\kappa}{4 \pi} \epsilon^{\mu \nu \lambda}\left(\partial_{\mu} \theta\right) \partial_{\nu}\left(\partial_{\lambda} \theta\right) .
\end{gathered}
$$

The Chern-Simons action $\mathcal{L}_{C S}$ can be rewritten using an auxiliary vector gauge field $d_{\mu}$,

$$
Z_{X Y}^{C S}:=\int \mathcal{D}[\theta] \mathcal{D}\left[d_{\mu}\right] \exp \left(i \int d^{3} x\left(\mathcal{L}_{X Y}[\theta]+\mathcal{L}_{d}\left[\theta, d_{\mu}\right]\right)\right),
$$

with

$$
\mathcal{L}_{d}\left[\theta, d_{\mu}\right]:=\frac{\kappa}{4 \pi} \epsilon^{\mu \nu \lambda} d_{\mu} \partial_{\nu} d_{\lambda}+\frac{\kappa}{2 \pi} d_{\mu} \epsilon^{\mu \nu \lambda} \partial_{\nu} \partial_{\lambda} \theta .
$$

Observe that the Chern-Simons gauge field $d_{\mu}$ couples to the current,

$$
\bar{j}_{\mathrm{vrt}}^{\mu} \equiv \frac{1}{2 \pi} \epsilon^{\mu \nu \lambda} \partial_{\nu} \partial_{\lambda} \theta
$$

This current is necessarily conserved,

$$
0=\partial_{\mu} \bar{j}_{\mathrm{vrt}}^{\mu} .
$$

We wish to constrain all configurations $\theta$ appearing in the partition function $Z_{X Y}^{C S}$ by condition (B4), i.e., we wish to restrict $\theta$ to any configuration such that it supports the conserved current $\bar{j}_{\mathrm{vrt}}^{\mu}$. We call such configurations vortex configurations.

The condition of current conservation (B4) can be enforced by the three Lagrange multipliers $c_{\mu} \mu=0,1,2$. If so, the following partition function restricted to vortex configurations follows:

$$
Z_{X Y \mathrm{vrt}}^{C S}:=\int \mathcal{D}[\theta] \mathcal{D}\left[c_{\mu}\right] \mathcal{D}\left[d_{\mu}\right] \exp \left(i \int d^{3} x \mathcal{L}_{X Y_{\mathrm{Vrt}}}^{C S}\left[\theta, c_{\mu}, d_{\mu}\right]\right),
$$

with

$$
\begin{aligned}
\mathcal{L}_{X Y \mathrm{vrt}}^{C S}\left[\theta, c_{\mu}, d_{\mu}\right]= & \frac{K}{2}\left(\partial_{\mu} \theta\right)\left(\partial^{\mu} \theta\right)+\frac{\kappa}{4 \pi} \epsilon^{\mu \nu \lambda} d_{\mu} \partial_{\nu} d_{\lambda}+\kappa d_{\mu} \bar{j}_{\mathrm{vrt}}^{\mu} \\
& -\frac{1}{2 \pi}{ }^{*} f^{\lambda} \partial_{\lambda} \theta+c_{\mu} \bar{j}_{\mathrm{vrt}}^{\mu} .
\end{aligned}
$$

Here, we have introduced the field

$$
{ }^{*} f^{\lambda} \equiv \epsilon^{\lambda \nu \mu} \partial_{\nu} c_{\mu},
$$

whose dual field is given by

$$
f_{\mu \nu}=\epsilon_{\mu \nu \lambda} * f^{\lambda}=\partial_{\mu} c_{\nu}-\partial_{\nu} c_{\mu},
$$

and we dropped total derivatives after performing partial integrations.

The equation of motion for $\theta$ gives the condition

$$
\partial^{\mu} \theta=\frac{1}{2 \pi K} * f^{\mu},
$$

from which we recover the inhomogeneous Maxwell equation,

$$
\bar{j}_{\mathrm{vrt}}^{\mu}=\frac{1}{4 \pi^{2} K} \partial_{\nu} f^{\mu \nu} .
$$

After integration over $\theta$, the partition function (B5a) and (B5b) becomes

$$
Z_{X Y \mathrm{vrt}}^{C S}=\int \mathcal{D}\left[c_{\mu}\right] \mathcal{D}\left[d_{\mu}\right] \exp \left(i \int d^{3} x \mathcal{L}_{\mathrm{vrt}}^{C S}\left[c_{\mu}, d_{\mu}\right]\right),
$$

with

$$
\begin{aligned}
\mathcal{L}_{\mathrm{vrt}}^{C S}\left[c_{\mu}, d_{\mu}\right]= & \frac{\kappa}{4 \pi} \epsilon^{\mu \nu \lambda} d_{\mu} \partial_{\nu} d_{\lambda}+\kappa d_{\mu} \bar{j}_{\mathrm{vrt}}^{\mu}-\frac{1}{16 \pi^{2} K} f^{\mu \nu} f_{\mu \nu} \\
& +c_{\mu} \bar{j}_{\mathrm{vrt}}^{\mu} .
\end{aligned}
$$

The dynamical gauge fields $c_{\mu}$ and $d_{\mu}$ that couple to the vortex current $j_{\text {vrt }}^{\mu}$ have a Maxwell for $c_{\mu}$ and Chern-Simons for $d_{\mu}$ kinetic energy. They endow the quantum theory with an explicit $\mathrm{U}(1) \times \mathrm{U}(1)$ local gauge symmetry.

\section{Exchange statistics}

We turn our attention to the computation of the exchange statistics of vortices with current $\bar{j}_{\mathrm{vrt}}^{\mu}$ interacting through the Chern-Simons action,

$$
\mathcal{L}_{\text {eff }}\left[d_{\mu}\right]:=\frac{\kappa}{4 \pi}\left(\epsilon^{\mu \nu \lambda} d_{\mu} \partial_{\nu} d_{\lambda}+4 \pi d_{\mu} \bar{j}_{\mathrm{vrt}}^{\mu}\right) .
$$

The relationship between the current and the field that results from the equations of motion is 


$$
\bar{j}_{\mathrm{vrt}}^{\mu}=-\frac{1}{2 \pi} \epsilon^{\mu \nu \lambda} \partial_{\nu} d_{\lambda}
$$

Hence, the vorticity

$$
n_{\theta}=\int d^{2} \boldsymbol{r} \bar{j}_{\mathrm{vrt}}^{0}(\boldsymbol{r})
$$

supported by the vortex current $\bar{j}_{\mathrm{vrt}}^{\mu}$ is related to the circulation from the gauge potential $d_{\mu}$ through

$$
n_{\theta}=-\frac{1}{2 \pi} \int d^{2} \boldsymbol{r}\left(\partial_{1} d_{2}-\partial_{2} d_{1}\right)=-\frac{1}{2 \pi} \oint d \boldsymbol{l} \cdot \boldsymbol{d} .
$$

Consider now winding two vortices, with vorticities $n_{1}$ and $n_{2}$ around each other. Without loss of generality, suppose that we hold vortex 1 at the location $x_{1}$ fixed and move vortex 2 along any closed trajectory $\boldsymbol{x}_{2}(t)$ that encircles once $\boldsymbol{x}_{1}$. On the one hand, the field $d_{\mu}^{(1)}\left(\boldsymbol{x}_{2}\right)$ at the location of vortex 2 that is induced by vortex 1 must then satisfy, according to Eq. (B13),

$$
\oint d \boldsymbol{x}_{2} \cdot \boldsymbol{d}^{(1)}\left(\boldsymbol{x}_{2}\right)=-2 \pi n_{1} .
$$

On the other hand, the vector current resulting from moving vortex 2 around vortex 1 is

$$
\overline{\boldsymbol{j}}_{\mathrm{vrt}}^{(2)}(t, \boldsymbol{x})=n_{2} \frac{d \boldsymbol{x}_{2}}{d t} \delta\left[\boldsymbol{x}-\boldsymbol{x}_{2}(t)\right] .
$$

Finally, the Berry phase acquired by winding vortex 2 around vortex 1 is

$$
\begin{aligned}
2 \Theta & =\frac{\kappa}{4 \pi}\left(4 \pi n_{2} \int d t \boldsymbol{d}^{(1)} \cdot \frac{d \boldsymbol{x}_{2}}{d t}\right) \\
& =\frac{\kappa}{4 \pi}\left(4 \pi n_{2} \oint d \boldsymbol{x}_{2} \cdot \boldsymbol{d}^{(1)}\right) \\
& =\frac{\kappa}{4 \pi}\left(-8 \pi^{2} n_{1} n_{2}\right) .
\end{aligned}
$$

We conclude that the statistical phase $\Theta$, which is one-half of the Berry phase, is given by

$$
\frac{\Theta}{\pi}=-\kappa n_{1} n_{2} .
$$

In particular, for a positive unit vortex winding around a negative unit vortex (antivortex), we find the statistical phase

$$
\frac{\Theta}{\pi}=\kappa \text {. }
$$

\section{APPENDIX C: BERRY PHASE IN THE SINGLE- PARTICLE APPROXIMATION}

We are going to describe how Aharonov-Bohm phases $\gamma$ or, more generally, Berry phases $\Theta$ accumulated under the pairwise exchanges of quasi-particles can be computed for noninteracting models of fermions defined on lattices. We first discuss Berry phases for lattice models of noninteracting fermions in all generality. We then specialize to the case of the $\pi$ flux phase for which we define vortices, axial gauge half fluxes, etc.

\section{Berry phase on the lattice}

Assume that we are given a lattice model, whose sites $\boldsymbol{r}$ and internal degrees of freedom are collectively denoted by the latin index $m$, that describes the quantum dynamics of noninteracting fermions. In second quantization, if the creation $\hat{c}_{m}^{\dagger}$ and annihilation $\hat{c}_{n}$ obey the usual fermion algebra,

$$
\left\{\hat{c}_{m}, \hat{c}_{n}^{\dagger}\right\}=\delta_{m, n}, \quad\left\{\hat{c}_{m}^{\dagger}, \hat{c}_{n}^{\dagger}\right\}=\left\{\hat{c}_{m}, \hat{c}_{n}\right\}=0,
$$

then we take our noninteracting Hamiltonian to be

$$
\hat{H}:=-\sum_{m, n} t_{m n} \hat{c}_{m}^{\dagger} \hat{c}_{n},
$$

where the matrix $t$ with the matrix elements $t_{m n}$ is Hermitian,

$$
t_{m n}=t_{n m}^{*} \text {. }
$$

We shall call the matrix $t$ the background. Its uniform diagonal matrix elements (the chemical potential) fixes the average number of fermions. We shall assume that some choices for the matrix $t$ can be associated with point-like defects. These pointlike defects can thus be labeled by their positions $\boldsymbol{r}_{1}, \boldsymbol{r}_{2}, \ldots$ on the lattice with their corresponding backgrounds $t_{\boldsymbol{r}_{1}, \boldsymbol{r}_{2}, \ldots}$. For a given filling fraction, the manybody ground state in the background $t_{\boldsymbol{r}_{1}, \boldsymbol{r}_{2}, \ldots}$ of pointlike defects is the Fermi sea,

$$
\left|t_{r_{1}, r_{2}, \ldots}\right\rangle:=\prod_{m}^{\prime} \hat{c}_{m}^{\dagger}|0\rangle .
$$

Here, the prime over the product means that only the lowest single-particle energy eigenstates are to be filled up to the given filling fraction out of the state $|0\rangle$ annihilated by the $\hat{c}_{m}$.

Imagine that we move the $k$ th pointlike defect along a closed path $\mathcal{P}_{k}$ of counterclockwise orientation while holding all other point-like defects fixed. We then discretize the path, thereby defining $N$ backgrounds $t_{\mathcal{P}_{k}}^{(n)}, n=1, \ldots, N$.

The gauge invariant phase $\gamma_{\mathcal{P}_{k}}$ is defined by

$$
\gamma_{\mathcal{P}_{k}}:=-\sum_{n=1}^{N} \arg \left\langle t_{\mathcal{P}_{k}}^{(n)} \mid t_{\mathcal{P}_{k}}^{(n+1)}\right\rangle \text {. }
$$

If we do this exercise for two cases, one when the path $\mathcal{P}_{k}$ encircles another defect $l$ and another one when the defect $l$ lies outside the path $\mathcal{P}_{k}$, we obtain the statistical phase $\Theta_{k l}$ acquired by the counterclockwise exchange of pointlike defects $k$ and $l$ from

$$
\Theta_{k l}:=\frac{1}{2}\left(\gamma_{l \text { inside } \mathcal{P}_{k}}-\gamma_{l \text { outside }} \mathcal{P}_{k}\right) .
$$

This phase does not depend on the presence of other static point-defect inside the path $\mathcal{P}_{k}$ for their contributions to $\gamma_{l \text { inside } \mathcal{P}_{k}}$ cancel their contributions to $\gamma_{l \text { outside } \mathcal{P}_{k}}$.

The overlaps 


$$
\Gamma_{n, n+1}:=\left\langle t_{\mathcal{P}_{k}}^{(n)} \mid t_{\mathcal{P}_{k}}^{(n+1)}\right\rangle
$$

from Eq. (C3) can be presented as the determinants for the products between two matrices built out of the eigenvectors of $t^{(n)}$ and $t^{(n+1)}$, as we now show. For any background $t$, define the unitary transformation $U$ by

$$
U t U^{\dagger}=\operatorname{diag}\left(\varepsilon_{m}\right),
$$

i.e., $U$ is the matrix of eigenvectors with energies $\varepsilon_{m}$ of the single-particle Hermitian matrix $t$. For the two backgrounds entering the overlap (C5), these unitary transformations are denoted by $U_{n}$ and $U_{n+1}$, respectively. One then verifies that

$$
\Gamma_{n, n+1}=\operatorname{det}\left(U_{n}^{\dagger} U_{n+1}\right) .
$$

Evaluation of phases $(\mathrm{C} 3)$ or $(\mathrm{C} 4)$ requires $N$ diagonalizations and the multiplication of $N$ determinants, a computing exercise that scales as a power law in the number of sites in the lattice.

\section{Lattice defects for the $\pi$-flux phase}

Consider a square Bravais lattice $\Lambda$ that is spanned by the orthogonal basis of vectors $s_{1}$ and $s_{2}$ of length $\mathfrak{a}$, the lattice spacing. We shall also define $\boldsymbol{s}_{3} \equiv-\boldsymbol{s}_{1}$ and $\boldsymbol{s}_{4} \equiv-\boldsymbol{s}_{2}$. The square lattice is the union of two interpenetrating square lattices $\Lambda_{\mathrm{A}}$ and $\Lambda_{\mathrm{B}}$ with lattice spacing $\sqrt{2} \mathfrak{a}$. Any site $\boldsymbol{r}_{\mathrm{B}} \in \Lambda_{\mathrm{B}}$ can be decomposed in a unique way according to $\boldsymbol{r}_{\mathrm{B}}=\boldsymbol{r}_{\mathrm{A}}$ $+\boldsymbol{s}_{1}$ with $\boldsymbol{r}_{\mathrm{A}} \in \Lambda_{\mathrm{A}}$.

Because of the bipartite nature of the square lattice, we introduce fermionic annihilation operators denoted $\hat{a}_{r_{\mathrm{A}}}$ and $\hat{b}_{\boldsymbol{r}_{\mathrm{B}}}$ and their adjoints for any site $\boldsymbol{r}_{\mathrm{A}}$ and $\boldsymbol{r}_{\mathrm{B}}$ of the sublattice $\Lambda_{\mathrm{A}}$ and $\Lambda_{\mathrm{B}}$, respectively. These operators obey the usual fermionic algebra with the only nonvanishing anticommutators,

$$
\left\{\hat{a}_{\boldsymbol{r}_{\mathrm{A}}}, \hat{a}_{\boldsymbol{r}_{\mathrm{A}}}^{\dagger}\right\}=1, \quad\left\{\hat{b}_{\boldsymbol{r}_{\mathrm{B}}}, \hat{b}_{\boldsymbol{r}_{\mathrm{B}}}^{\dagger}\right\}=1 .
$$

The square lattice with a flux of $\pi$ per plaquette (the $\pi$ flux phase in short) is the noninteracting tight-binding Hamiltonian,

$$
\hat{H}_{\pi}:=-\sum_{\boldsymbol{r} \in \Lambda_{\mathrm{A}}} \sum_{j=1}^{4}\left(t_{\boldsymbol{r}, \boldsymbol{r}+s_{j}}^{(\pi)} \hat{b}_{\boldsymbol{r}+s_{j}}^{\dagger} \hat{a}_{\boldsymbol{r}}+\text { H.c. }\right),
$$

with the (gauge dependent) choice of the tunneling amplitudes,

$$
\begin{gathered}
t_{\boldsymbol{r}, \boldsymbol{r}+s_{1}}^{(\pi)}=t_{\boldsymbol{r}, \boldsymbol{r}+s_{3}}^{(\pi)}=e^{i \pi / 2} t=i t, \\
t_{\boldsymbol{r}, \boldsymbol{r}+s_{2}}^{(\pi)}=t_{\boldsymbol{r}, \boldsymbol{r}+s_{4}}^{(\pi)}=t .
\end{gathered}
$$

Time-reversal symmetry is the property that $\hat{H}_{\pi}^{*}$ is locally gauge equivalent to $\hat{H}_{\pi}$. Sublattice symmetry is the property that $\hat{H}_{\pi} \rightarrow-\hat{H}_{\pi}$ under the local gauge transformation,

$$
\hat{a}_{r} \rightarrow+\hat{a}_{r}, \quad \hat{b}_{r+s_{1}} \rightarrow-\hat{b}_{r+s_{1}} .
$$

At half-filling, the Fermi surface collapses to two nonequivalent Fermi points due to the breaking of translation invariance for the unit cell is now the unit cell of the sublattice $\Lambda_{\mathrm{A}}$ with two atoms per unit cell. At half-filling, there are four non-equivalent ways to open a gap.

There is the charge-density wave instability through the perturbation

$$
\hat{H}_{\mu_{\mathrm{s}}}:=t \mu_{\mathrm{s}} \sum_{\boldsymbol{r} \in \Lambda_{\mathrm{A}}}\left(\hat{a}_{\boldsymbol{r}}^{\dagger} \hat{a}_{\boldsymbol{r}}-\hat{b}_{\boldsymbol{r}+s_{1}}^{\dagger} \hat{b}_{\boldsymbol{r}+s_{1}}\right)
$$

that breaks the sublattice symmetry $\hat{H}_{\pi} \rightarrow-\hat{H}_{\pi}$ of Hamiltonian $(\mathrm{C} 9 \mathrm{a})$ and $(\mathrm{C} 9 \mathrm{~b})$ under the local gauge transformation (C10) but preserves time-reversal symmetry, $\hat{H}_{\mu_{\mathrm{s}}}=\hat{H}_{\mu_{\mathrm{s}}}^{*}$.

There is the bond-density wave instability through the perturbation

$$
\hat{H}_{\Delta}:=-\sum_{\boldsymbol{r} \in \Lambda_{\mathrm{A}}} \sum_{j=1}^{4}\left(\delta t_{\boldsymbol{r}, \boldsymbol{r}+s_{j}} \hat{b}_{\boldsymbol{r}+s_{j}}^{\dagger} \hat{a}_{\boldsymbol{r}}+\text { H.c. }\right)
$$

with the tunneling amplitudes

$$
\begin{aligned}
\delta t_{\boldsymbol{r}, \boldsymbol{r}+\boldsymbol{s}_{j}}:= & \frac{t}{4}\left[i\left(\delta_{j, 1}+\delta_{j, 3}\right)+\left(\delta_{j, 2}+\delta_{j, 4}\right)\right] \\
& \times\left(\Delta e^{+i(\pi / 2) j} e^{+i \boldsymbol{G} \cdot \boldsymbol{r}}+\text { c.c. }\right),
\end{aligned}
$$

where the wave vector

$$
\boldsymbol{G}=\boldsymbol{K}_{+}-\boldsymbol{K}_{-}=\frac{\pi}{\mathfrak{a}}\left(\begin{array}{l}
1 \\
0
\end{array}\right)
$$

connects the two Fermi points

$$
K_{ \pm}:=\frac{\pi}{2 \mathfrak{a}}\left(\begin{array}{c} 
\pm 1 \\
1
\end{array}\right) .
$$

[Here, $\boldsymbol{G} \cdot \boldsymbol{r}=m_{1}+m_{2}$ if $\boldsymbol{r}=\left(m_{1} \boldsymbol{s}_{1}+m_{2} \boldsymbol{s}_{2}\right)$ with $m_{1}$ and $m_{2}$ integers.] It preserves the sublattice and time-reversal symmetries of $\hat{H}_{\pi}$. Notice that $\delta t_{r, r+s}$ are purely imaginary (real) when $j=1,3(j=2,4)$. When the complex-valued order parameter $\Delta$ is turned into a space-dependent order parameter $\Delta=\Delta_{0}(\boldsymbol{r}) e^{i f(\boldsymbol{r})}$ trough an amplitude $\Delta_{0}(\boldsymbol{r})$ and phase $f(\boldsymbol{r})$ modulation, then Eq. (C12b) turns into

$$
\begin{aligned}
\delta t_{\boldsymbol{r}, \boldsymbol{r}+s_{j}}= & \frac{t \Delta_{0}(\boldsymbol{r})}{2}\left[i\left(\delta_{j, 1}+\delta_{j, 3}\right)+\left(\delta_{j, 2}+\delta_{j, 4}\right)\right] \\
& \times \cos \left(f(\boldsymbol{r})+\frac{\pi}{2} j+\boldsymbol{G} \cdot \boldsymbol{r}\right) .
\end{aligned}
$$

If the bond-density wave supports the unit vortex $\Delta(\boldsymbol{r})$ $=\Delta_{0}(r) e^{ \pm i \theta}$ at the origin of the lattice, whereby we have introduced the polar coordinates $\boldsymbol{r} \cdot \boldsymbol{s}_{1} / \mathfrak{a}=r \cos \theta$ and $\boldsymbol{r} \cdot \boldsymbol{s}_{2} / \mathfrak{a}=r \sin \theta$, then

$$
\begin{aligned}
\delta t_{\boldsymbol{r}, \boldsymbol{r + s} j}= & \frac{t \Delta_{0}(r)}{2}\left[i\left(\delta_{j, 1}+\delta_{j, 3}\right)+\left(\delta_{j, 2}+\delta_{j, 4}\right)\right] \\
& \times \cos \left( \pm \theta+\frac{\pi}{2} j+\boldsymbol{G} \cdot \boldsymbol{r}\right) .
\end{aligned}
$$

The case of an arbitrary distribution of vortices of integer charges $n^{(k)}$ at the sites $\boldsymbol{r}^{(k)}$ follows with the identifications 


$$
f(\boldsymbol{r})=\sum_{k} n^{(k)} \arctan \frac{r_{2}-r_{2}^{(k)}}{r_{1}-r_{1}^{(k)}} .
$$

There is the time-reversal and sublattice symmetrybreaking bond-density wave,

$$
\begin{aligned}
H_{\eta}:= & -\sum_{\boldsymbol{r} \in \Lambda_{\mathrm{A}}} \sum_{j= \pm}\left(t_{2, j}^{a} \hat{a}_{\boldsymbol{r}+\boldsymbol{a}_{j}}^{\dagger} \hat{a}_{\boldsymbol{r}}+\text { H.c. }\right) \\
& -\sum_{\boldsymbol{r} \in \Lambda_{\mathrm{B}}} \sum_{j= \pm}\left(t_{2, j}^{b} \hat{b}_{\boldsymbol{r}+\boldsymbol{a}_{j}}^{\dagger} \hat{b}_{\boldsymbol{r}}+\text { H.c. }\right)
\end{aligned}
$$

where $\boldsymbol{a}_{ \pm}=\boldsymbol{s}_{1} \pm \boldsymbol{s}_{2}$ and

$$
t_{2,+}^{a}=t_{2,-}^{b}=+\frac{\eta}{4} t, \quad t_{2,-}^{a}=t_{2,+}^{b}=-\frac{\eta}{4} t .
$$

The lattice origin of the axial gauge field can also be identified with a staggered modulation of the nearestneighbor hopping through the perturbation

$$
\hat{H}_{5}:=-\sum_{r \in \Lambda_{\mathrm{A}}} \sum_{j=1}^{4}\left(\delta t_{r, \boldsymbol{r}+s_{j}}^{(5)} \hat{b}_{\boldsymbol{r}+s_{j}^{\dagger}}^{\dagger} \hat{a}_{\boldsymbol{r}}+\text { H.c. }\right),
$$

with

$$
\begin{aligned}
& +\delta t_{\boldsymbol{r}, \boldsymbol{r}+s_{2}}^{(5)}=-\delta t_{\boldsymbol{r}, \boldsymbol{r}+s_{4}}^{(5)} \equiv A_{1}^{(5)}(\boldsymbol{r}) t, \\
& -\delta t_{\boldsymbol{r}, \boldsymbol{r}+s_{1}}^{(5)}=+\delta t_{\boldsymbol{r}, \boldsymbol{r}+s_{3}}^{(5)} \equiv i A_{2}^{(5)}(\boldsymbol{r}) t .
\end{aligned}
$$

Motivated by the axial gauge flux

$$
a_{5 i}(\boldsymbol{r})=-n a(r) \epsilon_{i j} \frac{r^{j}}{r^{2}},
$$

where $a(r)$ is any function that vanishes no slower than $r$ at the origin and saturates to $1 / 2$ at infinity that screens a charge $n$ vortex in the continuum limit, we identify the lattice axial gauge flux that screens a charge $n$ vortex located at the origin with

$$
\begin{aligned}
& +\delta t_{r, \boldsymbol{r}+s_{2}}^{(5)}=-\delta t_{\boldsymbol{r}, \boldsymbol{r + s _ { 4 }}}^{(5)}=-t \frac{a(r)}{r} \sin \theta \\
& +\delta t_{r, \boldsymbol{r}+s_{1}}^{(5)}=-\delta t_{\boldsymbol{r}, \boldsymbol{r + s _ { 3 }}}^{(5)}=-i t \frac{a(r)}{r} \cos \theta
\end{aligned}
$$

where we choose to regularize the vortex with

$$
a(r)=\frac{1}{2} \tanh \frac{r}{\xi} .
$$

Here, $\xi$ is a characteristic length scale that determines the core radius of the axial gauge flux. The function $a(r)$ regularizes the singularity of $1 / r$ at the origin. The case of a distribution of axial gauge fluxes located at $\boldsymbol{r}^{(k)}$ follows with the substitutions $n \rightarrow n^{(k)}$ for the integer vortex charges, $\boldsymbol{r} \rightarrow \boldsymbol{r}-\boldsymbol{r}^{(k)}$ for the positions of the axial gauge fluxes, and a linear superposition of the corresponding tunneling amplitudes.

Finally, a uniform magnetic flux or a magnetic flux localized to one plaquette of the square lattice follows from the Peierls substitution

$$
t_{\boldsymbol{r}, \boldsymbol{r}+s_{j}}^{(\pi)} \rightarrow e^{i \phi_{\boldsymbol{r}, \boldsymbol{r}+s_{j}} t_{\boldsymbol{r}, \boldsymbol{r}+s_{j}}^{(\pi)}}
$$

in Eq. (C9b) with any suitable choice for the phases $\phi_{\boldsymbol{r}, \boldsymbol{r}+\boldsymbol{s}_{j}}$.

\section{APPENDIX D: CHIRAL SINGULAR GAUGE TRANSFORMATIONS}

The effective theory (4.13) is one of the main results of this paper. From it follows the charge and statistics of quasiparticles. This effective theory was derived by combining symmetry arguments to reach Eq. (4.10b) and a direct computation to fix the coefficients that symmetry does not determine. Computation of these coefficients can be achieved in many independent ways. For example, the computation of the coefficient $C_{03}^{(1)}$ is fixed by obtaining the charge of quasiparticles. Hence, $C_{03}^{(1)}$ can be deduced from Refs. 10, 15, and 16 for some range of the parameters $\mu_{\mathrm{s}}$ and $\eta$, or, more directly, from numerics. The key step to derive the effective theory (4.13) was the $\mathrm{U}(2)$ pure gauge transformation (4.1) [see also Eq. (3.10)]. In this Appendix, we compare these different ways of deriving effective actions for computing charge and statistics.

We consider the field theory defined by the partition function,

$$
\begin{gathered}
Z\left[a_{\mu}, a_{5 \mu}, \theta\right]:=\int \mathcal{D}[\bar{\psi}, \psi] \exp \left(i \int d^{3} x \mathcal{L}\right), \\
\mathcal{L}:=\bar{\psi}\left(i \partial-\not \iota-d_{5} \gamma^{5}-|\Delta| e^{i \theta \gamma^{5}}-\mu_{\mathrm{s}} R\right) \psi .
\end{gathered}
$$

We recognize Eq. (3.1a), (3.1b), (3.1c), and (3.1d) whereby contraction with the $4 \times 4$-dimensional gamma matrices is implied by the Feynman slash notation and there is no TRSbreaking (Haldane) mass $\eta$.

Following Seradjeh and Franz in Ref. 17, we perform the family of chiral gauge transformations

$$
\begin{aligned}
& \bar{\psi}=: \bar{\chi}_{\zeta} e^{-i \theta \gamma_{5} / 2} e^{+i(\zeta-1 / 2) \theta}, \\
& \psi=: e^{-i \theta \gamma_{5} / 2} e^{-i(\zeta-1 / 2) \theta} \chi_{\zeta} .
\end{aligned}
$$

The parameter $0 \leqslant \zeta \leqslant 1$ implements a choice of "partition" in the terminology of Ref. 17. Each chiral transformation (D2) is singular if the phase $\theta$ supports vortices; otherwise it is a pure gauge transformation. The (classical) transformation law of $\mathcal{L}$ in Eq. (D1) under the family of chiral transformations (D2) is

$$
\mathcal{L} \rightarrow \mathcal{L}_{\zeta}
$$

where

$$
\mathcal{L}_{\zeta}=\bar{\psi}\left(i \not b-d_{\zeta}-b \gamma^{5}-|\Delta|-\mu_{\mathrm{s}} R\right) \psi
$$

and

$$
d_{\zeta}=a-\left(\zeta-\frac{1}{2}\right) \partial \theta, \quad b=d_{5}-\frac{1}{2} \partial \theta .
$$

Observe that, whenever $\theta$ supports vortices and $\zeta \neq 1 / 2$, a physical magnetic flux has appeared where there was none to 
begin with. Thus, if we demand TRS, we must choose $\zeta=1 / 2$ and demand that $a_{\mu}$ is pure gauge.

In the spirit of Ref. 17, for general $\zeta$ we define the family of partition functions

$$
\begin{aligned}
Z_{\zeta}\left[a_{\zeta \mu}, b_{\mu}\right]: & =\int \mathcal{D}\left[\bar{\chi}_{\zeta}, \chi_{\zeta}\right] \exp \left(i \int d^{3} x \mathcal{L}_{\zeta}\right) \\
& \equiv \exp \left(i \int d^{3} x \mathcal{L}_{\zeta}^{\mathrm{eff}}\right)
\end{aligned}
$$

and compute the effective action $\mathcal{L}_{\zeta}^{\text {eff }}$ for the gauge fields $a_{\zeta \mu}$ and $b_{\mu}$ that follows from integrating the massive fermions $\bar{\chi}_{\zeta}$ and $\chi_{\zeta}$ to lowest order in a gradient expansion. Here, it should be noted that when the change of variables (D2) is singular, while the chiral anomaly is absent in $(2+1)$ dimensions, ${ }^{36}$ it can cost nontrivial Fujikawa Jacobian ${ }^{84}$ All ultraviolet divergences induced by the integration over the fermions can be disposed of with the help of the Pauli-Villars regularization scheme. The effective action, expressed in terms of $a_{\mu}, a_{5 \mu}$, and $\theta$, which follows to leading order in a gradient expansion, is

$$
\begin{aligned}
\mathcal{L}_{\zeta}^{\mathrm{eff}}= & \frac{|\Delta|^{2}}{2 \pi m}\left(a_{5 \mu}-\frac{1}{2} \partial_{\mu} \theta\right)\left(a_{5}^{\mu}-\frac{1}{2} \partial^{\mu} \theta\right) \\
& -\frac{2 Q}{2 \pi} \epsilon^{\nu \rho \kappa}\left(a_{\nu}-\frac{2 \zeta-1}{2} \partial_{\nu} \theta\right) \partial_{\rho}\left(a_{5 \kappa}-\frac{1}{2} \partial_{\kappa} \theta\right),
\end{aligned}
$$

where $Q=\operatorname{sgn} \mu_{\mathrm{s}} \frac{1}{2}\left(1-\mu_{\mathrm{s}} / m\right)$. The effective action (D5) fails to capture the charge of screened quasiparticles. For example, the conserved induced fermionic current,

$$
j_{\zeta}^{\mu}:=\frac{2 Q}{2 \pi} \epsilon^{\mu \rho \kappa} \partial_{\rho}\left(a_{5 \kappa}-\frac{1}{2} \partial_{\kappa} \theta\right),
$$

which is independent of the parameter $\zeta$, does not reproduce the induced fermionic charge (5.7) when the axial gauge field screens the mass vortices. It follows from their result that the charge bound to screened vortices (in which case $\left.a_{5 \kappa}-\frac{1}{2} \partial_{\kappa} \theta=0\right)$ is $Q=0$ instead of $Q=1 / 2$ as found in Refs. 15 and 16 , and in Sec. V.

Moreover, after proper dualization of the effective action (D5) [this dualization must include the Higgs mass, i.e., the first line on the right-hand side of Eq. (D5), a fact that was ignored in Ref. 17] it follows that the exchange statistics is $\zeta$ dependent. This is expected in view of the introduction of magnetic fluxes whenever $\zeta \neq 1 / 2$ contrary to the implicit assumption made in Ref. 17 when choosing $\zeta=0$.
${ }^{1}$ K. S. Novoselov, A. K. Geim, S. V. Morozov, D. Jiang, M. I. Katsnelson, I. V. Grigorieva, S. V. Dubonos, and A. A. Firsov, Nature (London) 438, 197 (2005).

${ }^{2}$ Y. Zhang, Y.-W. Tan, H. L. Stormer, and P. Kim, Nature (London) 438, 201 (2005).

${ }^{3}$ R. R. Nair, P. Blake, A. N. Grigorenko, K. S. Novoselov, T. J. Booth, T. Stauber, N. M. R. Peres, and A. K. Geim, Science 320, 1308 (2008).

${ }^{4}$ S. Y. Zhou, G.-H. Gweon, A. V. Fedorov, P. N. First, W. A. de Heer, D.-H. Lee, F. Guinea, A. H. Castro Neto, and A. Lanzara, Nature Mater. 6, 770 (2007); 6, 916 (2007).

${ }^{5}$ Joseph G. Checkelsky, Lu Li, and N. P. Ong, Phys. Rev. B 79, 115434 (2009).

${ }^{6}$ K. Nomura, S. Ryu, and D. Lee, arXiv:0906.0159 (unpublished).

${ }^{7}$ C.-Y. Hou, C. Chamon, and C. Mudry arXiv:0909.2984 (unpublished).

${ }^{8}$ G. W. Semenoff, Phys. Rev. Lett. 53, 2449 (1984).

${ }^{9}$ F. D. M. Haldane, Phys. Rev. Lett. 61, 2015 (1988).

${ }^{10}$ C.-Y. Hou, C. Chamon, and C. Mudry, Phys. Rev. Lett. 98, 186809 (2007).

${ }^{11}$ C. G. Callan, Jr. and J. A. Harvey, Nucl. Phys. B 250, 427 (1985).

${ }^{12}$ R. Jackiw and C. Rebbi, Phys. Rev. D 13, 3398 (1976).

${ }^{13}$ W. P. Su, J. R. Schrieffer, and A. J. Heeger, Phys. Rev. Lett. 42, 1698 (1979); Phys. Rev. B 22, 2099 (1980).

${ }^{14}$ R. Jackiw and J. R. Schrieffer, Nucl. Phys. B 190, 253 (1981).

${ }^{15}$ C. Chamon, C.-Y. Hou, R. Jackiw, C. Mudry, S.-Y. Pi, and A. P. Schnyder, Phys. Rev. Lett. 100, 110405 (2008).

${ }^{16}$ C. Chamon, C.-Y. Hou, R. Jackiw, C. Mudry, S.-Y. Pi, and G.
Semenoff, Phys. Rev. B 77, 235431 (2008).

${ }^{17}$ B. Seradjeh and M. Franz, Phys. Rev. Lett. 101, 146401 (2008).

${ }^{18}$ M. V. Milovanovic, Phys. Rev. B 78, 245424 (2008).

${ }^{19}$ R. Jackiw and S.-Y. Pi, Phys. Rev. Lett. 98, 266402 (2007).

${ }^{20}$ The gradient $\boldsymbol{\partial} \equiv \frac{\partial}{\partial \boldsymbol{r}}$ and the time derivative $\partial_{t} \equiv \frac{\partial}{\partial t}$ form the covariant three-vector $\partial_{\mu}=\left(\partial_{t}, \boldsymbol{\partial}\right)$.

${ }^{21}$ C. Itzykson and J.-B. Zuber, Quantum Field Theory (McGrawHill, New York, 1980).

${ }^{22}$ J. W. McClure, Phys. Rev. 104, 666 (1956).

${ }^{23}$ S. V. Morozov K. S. Novoselov, M. I. Katsnelson, F. Schedin, L. A. Ponomarenko, D. Jiang, and A. K. Geim, Phys. Rev. Lett. 97, 016801 (2006).

${ }^{24}$ A. F. Morpurgo and F. Guinea, Phys. Rev. Lett. 97, 196804 (2006).

${ }^{25}$ J. Gonzalez, F. Guinea, and M. A. H. Vozmediano, Phys. Rev. Lett. 69, 172 (1992); Nucl. Phys. B 406, 771 (1993).

${ }^{26}$ P. E. Lammert and V. H. Crespi, Phys. Rev. Lett. 85, 5190 (2000).

${ }^{27}$ J. K. Pachos, M. Stone, and K. Temme, Phys. Rev. Lett. 100, 156806 (2008).

${ }^{28}$ I. K. Affleck and J. B. Marston, Phys. Rev. B 37, 3774 (1988).

${ }^{29}$ X. G. Wen, F. Wilczek, and A. Zee, Phys. Rev. B 39, 11413 (1989).

${ }^{30}$ E. Fradkin, Field Theories of Condensed Matter Systems (Addison-Wesley, Redwood City, CA, 1991).

${ }^{31}$ C. Mudry and E. Fradkin, Phys. Rev. B 49, 5200 (1994); 50, 11409 (1994).

${ }^{32}$ A. W. W. Ludwig, M. P. A. Fisher, R. Shankar, and G. Grinstein, Phys. Rev. B 50, 7526 (1994). 
${ }^{33}$ Y. Hatsugai, X.-G. Wen, and M. Kohmoto, Phys. Rev. B 56, 1061 (1997).

${ }^{34}$ S. Guruswamy, A. LeClair, and A. W. W. Ludwig, Nucl. Phys. B 583, 475 (2000).

${ }^{35}$ The microscopic tight-binding model can be represented by a real-valued symmetric Hamiltonian.

${ }^{36}$ K. Fujikawa and H. Suzuki, Path Integrals and Quantum Anomalies (Oxford University Press, Oxford, 2004).

${ }^{37}$ T. Jaroszewicz, Phys. Lett. B 146, 337 (1984).

${ }^{38}$ Y.-H. Chen and F. Wilczek, Int. J. Mod. Phys. B 3, 117 (1989).

${ }^{39}$ Z. Hlousek, D. Senechal, and S. H. Henry Tye, Phys. Rev. D 41, 3773 (1990).

${ }^{40}$ V. M. Yakovenko, Phys. Rev. Lett. 65, 251 (1990).

${ }^{41}$ A. G. Abanov and P. B. Wiegmann, Nucl. Phys. B 570, 685 (2000).

${ }^{42}$ T. Jaroszewicz, Phys. Rev. D 34, 3128 (1986).

${ }^{43}$ S. Deser, R. Jackiw, and S. Templeton, Ann. Phys. (N.Y.) 140, 372 (1982).

${ }^{44}$ M. Blau and G. Thompson, Ann. Phys. (N.Y.) 205, 130 (1991).

${ }^{45}$ T. H. Hansson, Vadim Oganesyan, and S. L. Sondhi, Ann. Phys. (N.Y.) 313, 497 (2004).

${ }^{46}$ B. Seradjeh, C. Weeks, and M. Franz, Phys. Rev. B 77, 033104 (2008).

${ }^{47}$ K. Wilson in New Phenomena in Subnuclear Physics, edited by A. Zichichi (Plenum, New York, 1977).

${ }^{48}$ M. Levin and X.-G. Wen, Phys. Rev. B 67, 245316 (2003).

${ }^{49}$ C. L. Kane and E. J. Mele, Phys. Rev. Lett. 95, 146802 (2005).

${ }^{50}$ C. W. J. Beenakker, Phys. Rev. Lett. 97, 067007 (2006).

${ }^{51}$ Bruno Uchoa and A. H. Castro Neto, Phys. Rev. Lett. 98, 146801 (2007).

${ }^{52}$ P. Ghaemi and F. Wilczek, arXiv:0709.2626 (unpublished).

${ }^{53}$ Doron L. Bergman and Karyn Le Hur, Phys. Rev. B 79, 184520 (2009).

${ }^{54}$ R. Roy, arXiv:cond-mat/0608064 (unpublished).

${ }^{55}$ Andreas P. Schnyder, Shinsei Ryu, Akira Furusaki, and Andreas W. W. Ludwig, Phys. Rev. B 78, 195125 (2008).

${ }^{56}$ R. Roy, arXiv:0803.2868 (unpublished).

${ }^{57}$ Xiao-Liang Qi, Taylor L. Hughes, Srinivas Raghu, and ShouCheng Zhang, Phys. Rev. Lett. 102, 187001 (2009).

${ }^{58}$ A. Yu. Kitaev, Periodic table for topological insulators and superconductors (http://landau100.itp.ac.ru/Talks/kitaev.pdf).

${ }^{59}$ A. Tanaka and X. Hu, Phys. Rev. Lett. 95, 036402 (2005).

${ }^{60}$ Akihiro Tanaka and Xiao Hu, Phys. Rev. B 74, 140407 (2006).
${ }^{61}$ P. Ghaemi, S. Ryu, and D. Lee, arXiv:0903.1662 (unpublished).

${ }^{62}$ I. F. Herbut, Phys. Rev. Lett. 99, 206404 (2007).

${ }^{63}$ T. Senthil, A. Vishwanath, L. Balents, S. Sachdev, and M. P. A. Fisher, Science 303, 1490 (2004).

${ }^{64}$ T. Senthil, L. Balents, S. Sachdev, A. Vishwanath, and M. P. A. Fisher, Phys. Rev. B 70, 144407 (2004).

${ }^{65}$ T. Senthil and Matthew P. A. Fisher, Phys. Rev. B 74, 064405 (2006).

${ }^{66}$ Y. Ran and X. Wen, Phys. Rev. Lett. 96, 026802 (2006); arXiv:cond-mat/0609620 (unpublished).

${ }^{67}$ Anders W. Sandvik, Phys. Rev. Lett. 98, 227202 (2007).

${ }^{68}$ Roger G. Melko, and K. Kaul, Phys. Rev. Lett. 100, 017203 (2008).

${ }^{69}$ Ribhu K. Kaul, Roger G. Melko, Max A. Metlitski, and Subir Sachdev, Phys. Rev. Lett. 101, 187206 (2008).

${ }^{70}$ Tarun Grover and T. Senthil, Phys. Rev. Lett. 100, 156804 (2008).

${ }^{71}$ Ying Ran, Ashvin Vishwanath, and Dung-Hai Lee, Phys. Rev. Lett. 101, 086801 (2008).

${ }^{72}$ Xiao-Liang Qi and Shou-Cheng Zhang, Phys. Rev. Lett. 101, 086802 (2008).

${ }^{73}$ B. Andrei Bernevig, Taylor L. Hughes, and Shou-Cheng Zhang, Science 314, 1757 (2006).

${ }^{74}$ Markus König, Steffen Wiedmann, Christoph Bruene, Andreas Roth, Hartmut Buhmann, Laurens W. Molenkamp, Xiao-Liang Qi, and Shou-Cheng Zhang, Science 318, 766 (2007).

${ }^{75}$ Markus König, Hartmut Buhmann, Laurens W. Molenkamp, Taylor L. Hughes, Chao-Xing Liu, Xiao-Liang Qi, and ShouCheng Zhang, J. Phys. Soc. Jpn. 77, 031007 (2008).

${ }^{76}$ Xiao-Liang Qi, Taylor L. Hughes, and Shou-Cheng Zhang, Nat. Phys. 4, 273 (2008).

${ }^{77}$ X. G. Wen and A. Zee, Phys. Rev. B 44, 274 (1991).

${ }^{78}$ Equation (7) in Ref. 15 and the heuristic argument that follows are both wrong.

${ }^{79}$ T. Banks, R. J. Myerson, and J. Kogut, Nucl. Phys. B 129, 493 (1977).

${ }^{80}$ P. R. Thomas and M. Stone, Nucl. Phys. B 144, 513 (1978).

${ }^{81}$ M. Peskin, Ann. Phys. (N.Y.) 113, 122 (1978).

${ }^{82}$ C. Dasgupta and B. I. Halperin, Phys. Rev. Lett. 47, 1556 (1981).

${ }^{83}$ M. P. A. Fisher and D. H. Lee, Phys. Rev. B 39, 2756 (1989).

${ }^{84}$ C. D. Fosco and F. A. Schaposnik, Phys. Lett. B 477, 341 (2000). 